\title{
An Exploration of Emotional Intelligence in Virtual Teams
}

\author{
By \\ Nathaniel Lupton \\ Bachelor of Commerce (2004)
}

\author{
A thesis submitted to the \\ Faculty of Graduate Studies and Research \\ in partial fulfillment \\ of the requirements for the degree of \\ Master of Business Administration
}

Eric Sprott School of Business

Carleton University

Ottawa, Canada

(C) 2006 Nathaniel Lupton

May 19, 2005 


$\begin{array}{ll}\begin{array}{l}\text { Library and } \\ \text { Archives Canada }\end{array} & \begin{array}{l}\text { Bibliothèque et } \\ \text { Archives Canada }\end{array} \\ \begin{array}{l}\text { Published Heritage } \\ \text { Branch }\end{array} & \begin{array}{l}\text { Direction du } \\ \text { Patrimoine de l'édition }\end{array} \\ \begin{array}{l}\text { 395 Wellington Street } \\ \text { Ottawa ON K1A ON4 }\end{array} & \begin{array}{l}\text { 395, rue Wellington } \\ \text { Ottawa ON K1A ON4 } \\ \text { Canada }\end{array}\end{array}$

Your file Votre référence ISBN: 978-0-494-18351-9 Our file Notre référence ISBN: 978-0-494-18351-9

NOTICE:

The author has granted a nonexclusive license allowing Library and Archives Canada to reproduce, publish, archive, preserve, conserve, communicate to the public by telecommunication or on the Internet, loan, distribute and sell theses worldwide, for commercial or noncommercial purposes, in microform, paper, electronic and/or any other formats.

The author retains copyright ownership and moral rights in this thesis. Neither the thesis nor substantial extracts from it may be printed or otherwise reproduced without the author's permission.
AVIS:

L'auteur a accordé une licence non exclusive permettant à la Bibliothèque et Archives Canada de reproduire, publier, archiver, sauvegarder, conserver, transmettre au public par télécommunication ou par l'Internet, prêter, distribuer et vendre des thèses partout dans le monde, à des fins commerciales ou autres, sur support microforme, papier, électronique et/ou autres formats.

L'auteur conserve la propriété du droit d'auteur et des droits moraux qui protège cette thèse. $\mathrm{Ni}$ la thèse ni des extraits substantiels de celle-ci ne doivent être imprimés ou autrement reproduits sans son autorisation.
In compliance with the Canadian

Privacy Act some supporting forms may have been removed from this thesis.

While these forms may be included in the document page count, their removal does not represent any loss of content from the thesis.
Conformément à la loi canadienne sur la protection de la vie privée, quelques formulaires secondaires ont été enlevés de cette thèse.

Bien que ces formulaires aient inclus dans la pagination, il n'y aura aucun contenu manquant.

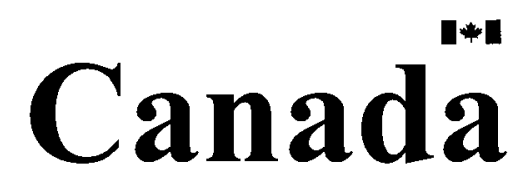




\begin{abstract}
Advanced technologies and the internet have enabled work arrangements not constrained by geographic proximity. One such work arrangement is the virtual team. This study explores the role of emotional intelligence (EI); the ability to perceive, use, understand and manage emotions in the self and others in a virtual team.

While EI research has provided substantial implications for leadership, quality of social interaction and performance in general, no known study examines the role of EI in virtual teams. Through a content analysis of a small virtual team's email archives over a two month period, and an assessment of individual differences in EI abilities, personality traits and general cognitive ability, it was found that these differences influence the communication style of email communicators. Furthermore, these effects were found to be moderated by the communicative intent of message senders.
\end{abstract}




\section{ACKNOWLEDGEMENTS}

The author gratefully acknowledges the support and expert guidance of thesis advisors Dr. Mike Hine and Dr. Steven Murphy of the Sprott School of Business as well as thesis board members Dr. Gerald Grant of the Sprott School of Business and Dr. John Zelenski of the Department of Psychology, all at Carleton University.

The author also wishes to express sincere gratitude to the individuals, who shall remain anonymous here, who graciously provided the data for this study. 


\section{TABLE OF CONTENTS}

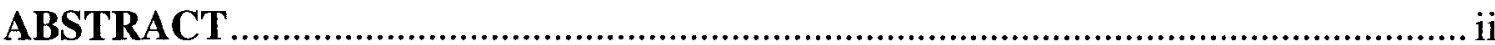

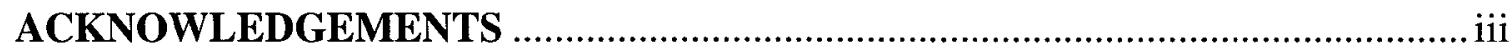

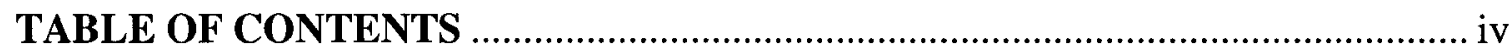

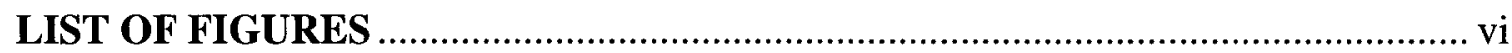

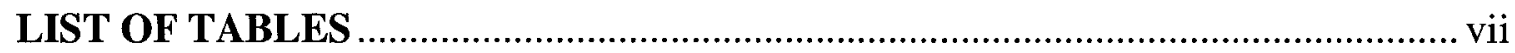

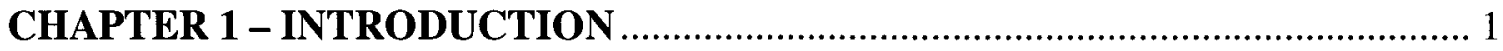

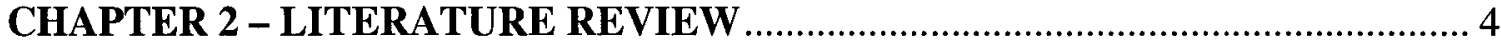

2.1 Virtual Work and Virtual Teams ............................................................................ 4

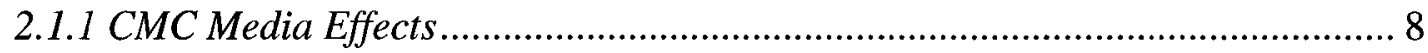

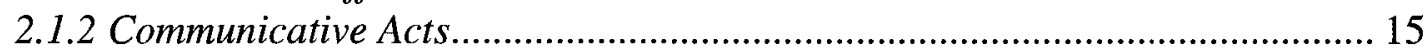

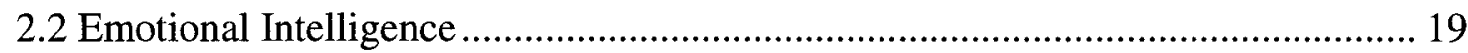

2.2.2 The Mayer Salovey Caruso Emotional Intelligence Test.............................. 21

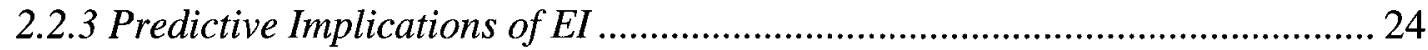

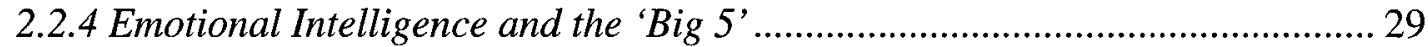

2. 3 Emotion, Emotional Intelligence, and (Virtual) Work ...................................... 36

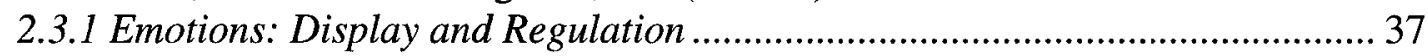

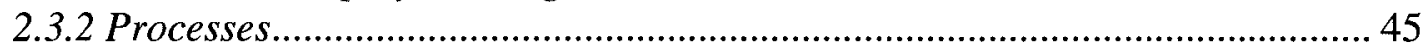

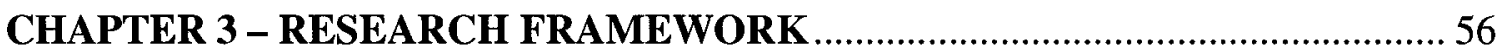

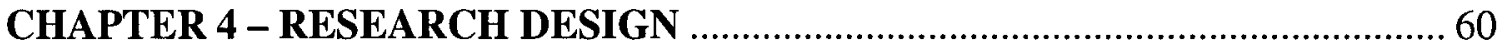

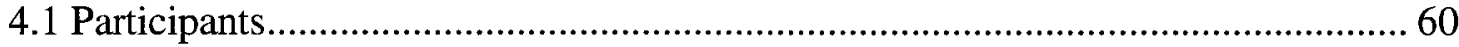

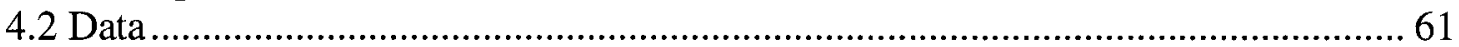

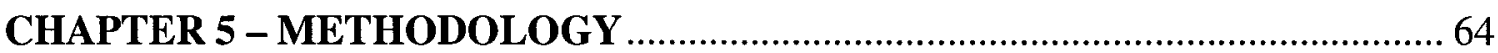

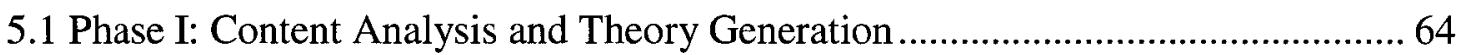

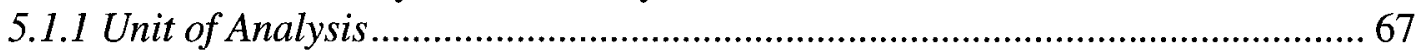

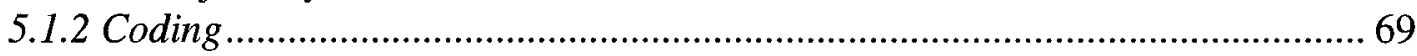

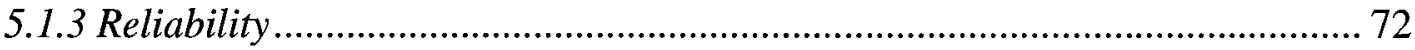

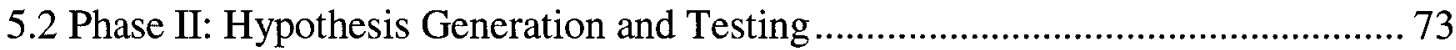

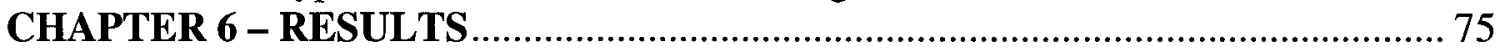

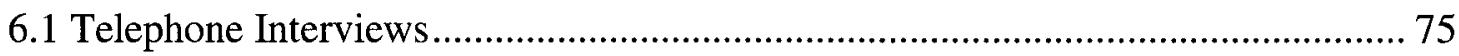

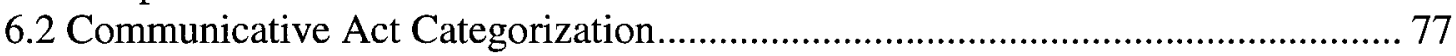

6.3 Communication Style Categorization .............................................................. 79

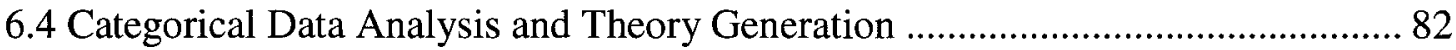

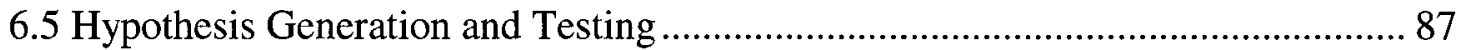

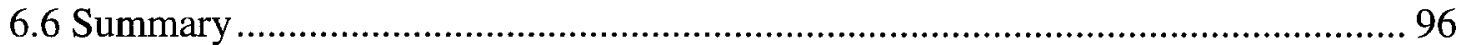

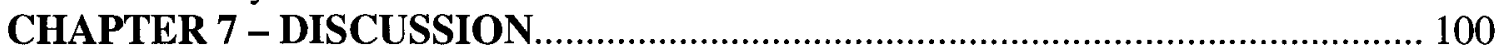

7.1 Findings Related to Personality Traits ........................................................... 100

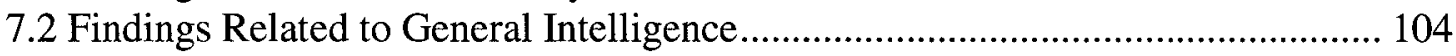

7.3 Findings Related to Emotional Intelligence....................................................... 105

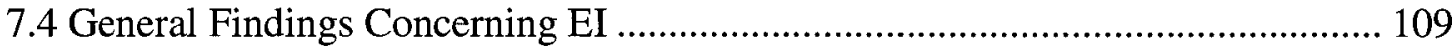

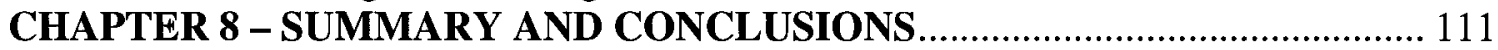

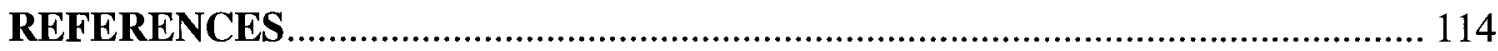

APPENDIX A - Big Five Inventory Items ................................................. 130 
APPENDIX B - Semi-Structured Interview Questions

APPENDIX C - Communicative Act Decision Tree

APPENDIX D - Communicative Style Decision Tree

APPENDIX E - Coder Instructions

APPENDIX F - Detailed MSCEIT Scores 


\section{LIST OF FIGURES}

Figure 1: Four Branch Ability Model of Emotional Intelligence. .................................. 22

Figure 2: Factors Involved in the Process of Creating Email Messages........................ 57

Figure 3: Model of Individual Difference Influence on Communicative Style................ 87 


\section{LIST OF TABLES}

Table 1: A comparison between Face to Face (FtF) and Computer-Mediated

Communication (CMC) characteristics. .............................................................. 10

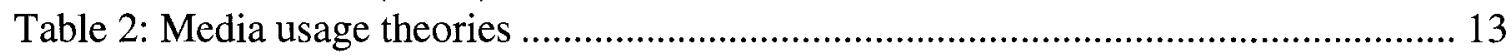

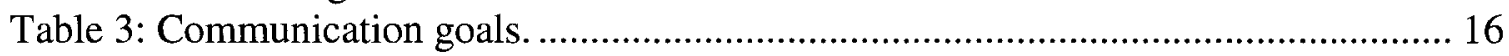

Table 4: Meta analysis of current research findings with respect to the correlation between mixed and self report measures of EI and the Big 5 personality traits...... 32

Table 5: Meta analysis of current research findings with respect to the correlation between ability-based measures of EI and the Big 5 personality traits ................... 33

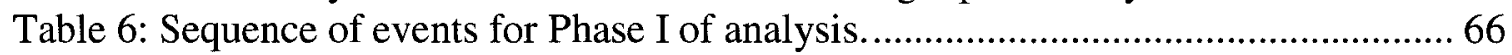

Table 7: Variables Available for Analysis in Second Phase.......................................... 73

Table 8: Sequence of events for Phase II analysis................................................ 73

Table 9: Communicative Act Rater Agreement........................................................... 78

Table 10: Communication Style Rater Agreement................................................... 82

Table 11: Communicative Act Proportions for Each Team Member............................ 83

Table 12: Communicative Style Proportions for Each Team Member............................ 83

Table 13: Proportion of Message Styles within Each Communicative Act (Chris)......... 84

Table 14: Proportion of Message Styles within Each Communicative Act (Brian) ......... 85

Table 15: Proportion of Message Styles within Each Communicative Act (Daniel) ...... 85

Table 16: Proportion of Message Styles within Each Communicative Act (John). ......... 85

Table 17: Proportion of Message Styles within Each Communicative Act (Jason)........ 85

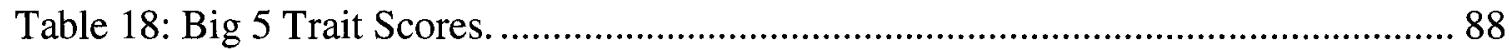

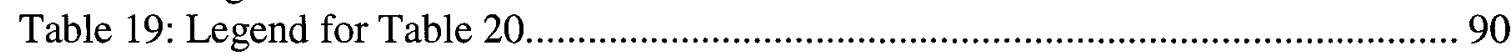

Table 20: Associations between Traits and Communicative Styles ............................... 91

Table 21: Legend for Table 22 ............................................................................ 92

Table 22: Associations between $\mathrm{G}$ and Communicative Styles................................. 93

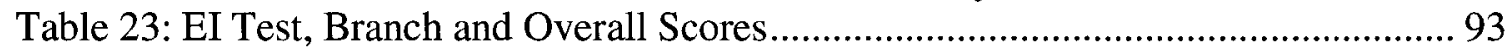

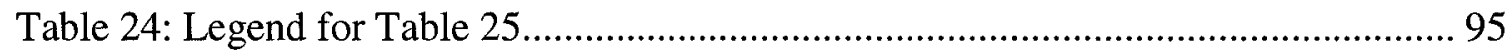

Table 25: Associations between EI Branch Scores and Communicative Styles .............. 96

Table 26: Summary of Significant Correlations ....................................................... 97

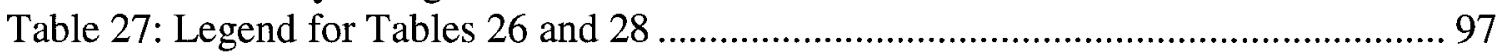

Table 28: Summary of Significant Regressions....................................................... 98 


\section{CHAPTER 1 - INTRODUCTION}

Virtual teams are an increasingly common work structure in contemporary organizations. Enabled by computer mediated communication (CMC) and advanced technologies, organizations are able to bring together human talent separated by time zones and geographic distance. Another domain which has captured the attention of researchers and practitioners alike is emotional intelligence (EI) which is the set of abilities enabling an individual to understand and manage self and others' emotions. EI enhances the ability of workers (and individuals in general) to perform well at tasks, disregard destructive in favour of constructive behaviours, and form more positive and lasting relationships with others.

EI has never been specifically examined in a virtual environment. This exploratory study used a grounded theory approach to seek evidence of emotional intelligence within the textual artefacts of a small virtual team. Specifically, email archives of a small virtual team were examined for evidence that EI influences the content of communication.

The participants in this study are the five members of a software development and support team funded by federal and state governments, operating from various locations in the Eastern United States. Each member of the team provides a distinct function and works at a distance from other members of the team. The team exhibits high task interdependence and is well coordinated. The primary communication medium used is

email. Although conference calls are sometimes used during events of major significance 
to the team, these calls occur rarely. The team is unusual in that it does not exist as part of a larger organizational entity. Literally, the team is the organization.

The goal of this study was to determine the extent to which EI abilities influence the content of email messages. The study begins by reviewing pertinent literature. In this review, virtual teams and the communication technologies on which they rely are introduced. Following this, the EI construct is defined, an overview of the MSCEIT model of EI is provided, the predictive implications of EI are discussed and the construct is related to the Big 5 personality traits. The review next draws upon relevant literature from Organizational Behaviour and Information Systems and synthesizes these into an overview of emotion, emotional intelligence and virtual work. Within this final section of the literature review, research areas examined include mood and emotion at work, emotional labour, group affect, job satisfaction, trust, conflict, and leadership.

Next, a preliminary research framework is presented to guide the subsequent inquiry. This framework summarizes relevant findings from the literature as they pertain to email message formation and suggests potential linkages between constructs. The design of the study, which employs facets of grounded theory and hermeneutics, as typically applied to the study of Information Systems, to a content analysis of email text is next presented. This content analysis results in the formulation of a theory in support of the study's objectives. To test this theory, several hypotheses were formulated and tested as described in the results section. In this phase of the study, the categorical results of the email content analysis were analyzed in conjunction with the other measures employed. These included EI scores, general intelligence, Big 5 personality traits, and the results of 
semi-structured interviews with each employee. The results of hypotheses testing are next discussed followed by limitations and directions for future research. 


\section{CHAPTER 2 - LITERATURE REVIEW}

A study on emotional intelligence in virtual teams is, by necessity, highly interdisciplinary. The Information Systems field has produced a vast body of knowledge on virtual teams. This knowledge draws heavily on Organizational Behaviour research for its relevant expertise in team performance and dynamics. One of the greatest dividing factors between virtual and conventional teams is the use of computer mediated communication (CMC) technologies as the primary coordination mechanism. This reality has spawned a significant body of knowledge in the Communication literature including a debate concerning the appropriateness of a medium. Within this literature we see indications of the implications of CMC use on emotional expressions (e.g. Rice \& Love, 1987). The study of emotion primarily belongs to the field of Psychology but also increasingly to Organizational Behaviour while EI belongs to the field of Organizational Behaviour.

\section{$\underline{2.1}$ Virtual Work and Virtual Teams}

The advent and subsequent proliferation of communication technologies in contemporary organizations has enabled work arrangements not constrained by the geographic proximity of workers. Communication technologies which enable such structures are by no means new. The invention of the telephone by Alexander Graham Bell in 1876 allowed individuals, potentially workers, to communicate from areas around the globe. Indeed email, which may be viewed as an extension of the telephone, appears 
to be the greatest driver of internet usage amongst home users (Kraut, Mukhopadhyay, Szczypula, Kiesler \& Scherlis, 1999). However, the proliferation of the internet and the various applications it supports coincides with an alteration in the means by which work is coordinated for an increasing number of organizations.

This technology, despite its enabling characteristics, is not the only driver of border-spanning work arrangements. Organizations competing in a global marketplace must seek out talented employees wherever they reside and tap into potential markets no matter how remote. This is no longer a competitive advantage for many firms; it is a competitive reality with survival at stake. Could economic globalization occur in the absence of the internet? Could global transactions and coordination of disparate organizational bodies take place using telephony alone? This study does not concern itself with such speculations. What has come to pass in the global economy may have been driven by the existence of newer technology or new technology may have been driven by an increased need for it. Without entering this precedence debate, we can at least acknowledge that contemporary forms of organization and communication may have now entered a new era (Barret, Jarvenpaa, Silva \& Walsham, 2003).

Distributed work structures enabled by CMC technologies are one form of organization enabled by technology. The advent of such organizational structures has given rise to terms such as 'virtual work', 'virtual team' and 'virtual organization'. Another virtual structure, not entirely related to contemporary organizations, is the 'virtual community'. Virtual work structures, of whatever form, are defined and bound by common goals and task interdependence, as in any work structure. However, in a virtual 
team or workgroup, members are geographically dispersed and communication is enabled by CMC technology (Gibson \& Cohen, 2003; Lipnack \& Stamps, 1997).

Virtual communities possess less well defined goals and are typically not task interdependent but instead members share common interests (Butler, 2001; De Cindio, Gentile, Grew \& Redolfi, 2003). The term 'virtual organization' is sometimes used interchangeably with 'virtual team' (Ahuja, M. \& Carley, K., 1999) in the Information Systems literature. However, it is also used to describe a structure in which individuals and organizational subunits from several organizations are linked via electronic networks to pursue goals and opportunities in a timely fashion (DeSanctis \& Monge, 1999; Grabowski \& Roberts, 1999). The motivation for forming such an organization is the lack of availability of resources such as skills, knowledge and information within a single organization. Virtual teams, which are analyzed in this case study, are typically contained within a single organizational body while maintaining geographic distribution.

Virtual teams are perhaps the most studied form of virtual organizing. The proliferation of virtual team literature in Information Systems research has resulted in the creation of several studies which organize and synthesize this vast body of knowledge (Hertel, Geister \& Konradt, 2005; Martins, Gilson \& Maynard, 2004; Pinsonneault \& Caya, 2005; Powell, Piccoli \& Ives, 2004). Technology enables virtual teams and provides the opportunity for organizations to address the changing work environment (Townsend, DeMarie, \& Hendrickson, 1998). A virtual team is distinguished from its non-virtual counterpart by its use of CMC technology as a primary communication medium and the geographic distribution of the individuals constituting the team. A non- 
virtual team, then, interacts through primarily face-to-face (FtF) communication and its members are co-located.

It has been suggested that virtuality should be viewed as a continuum (Leenders, vanEngelen \& Kratzer, 2003). In this view, a completely virtual team is one in which members communicate only through CMC technology and never meet FtF. A completely non-virtual team, then, inhabits the same workspace and never uses technology to communicate. Such teams may exist at opposite ends of the virtuality spectrum but most contemporary teams will finds themselves located somewhere in the middle.

Consider, for example, a large organization in which members have access to email. Even where members are not overtly constrained by physical distance, they often choose email to communicate with other organizational members. Also consider a large multinational corporation where physical and temporal distributions are more constraining. In this corporation, engineering functions are located in California while manufacturing is done in China. New product development is a joint task between these (and other) divisions of the company. Members of the engineering department are colocated but must also coordinate with members of the manufacturing division. There is a degree of virtuality introduced by these two co-located sub-teams in forming the new product development team in that $\mathrm{CMC}$ is the best available alternative to $\mathrm{FtF}$ interaction.

These two examples are presented to inject the reality of operating in modern organizations into the distinction between a virtual and non-virtual team. Certainly, various other examples exist which pull organization units from the extremes of the virtuality continuum by definition. 
The use of the term 'virtual' to describe certain work arrangements likely arises from other contemporary, technology based, applications such as 'virtual reality'. However, these uses of the term convey very different meanings. Virtual reality is a facsimile or representation of a physical reality enabled and created by advanced technology. This use of the term conveys a degree of separation between what is 'real' and what is 'virtual'. Virtual teams, however, are certainly not intended to be a facsimile but instead are entirely real (Lipnack \& Stamps, 1997). Virtual teams perform virtual work, and this work is also very real. While virtual team members are real people engaged in real work, the virtual environment is distinct on certain levels from co-located teams.

According to Burgoon et al. (2002), these differences include the effect of media characteristics, the effect of geographic distribution and level of communication richness. These distinctions fall into two major categories: the effect of the CMC medium, which includes communication richness as these dimensions are usually discussed together, and the effect of increased geographic distribution of virtual team members. The latter category is not examined here as the virtual team subject of this study does not experience temporal or cultural differences which lead to certain effects.

\subsubsection{CMC Media Effects}

Effective communication is imperative in coordinating the efforts of any team but is often considered more critical in the successful operation of virtual teams (DeSanctis \& Monge, 1999). Two general themes for studying CMC have arisen in the 
Communications and Information Systems literature. The first involves comparing media characteristics of $\mathrm{CMC}$ to the benchmark of FtF communication. This approach is labelled the 'media characteristics' approach in this study. The second considers media effects as less deterministic in the communication process. Under this approach, which shall be called the 'media adoption/adaptation' approach, users adapt their communication styles to the available medium in order to convey information which would be considered impossible, or at least inappropriate, by the media characteristics theories.

Researchers employing the media characteristics approach examine various media for their ability to convey aspects of FtF communication thereby suggesting what 'can and cannot' be conveyed in CMC. The most well known and often cited theory within this category is Media Richness Theory (MRT: Daft \& Lengel., 1986). Richness here refers to the ability of the media to accurately convey ambiguous information. Rice (1993) adds to this definition of richness the ability to convey social presence of the communicators. According to MRT, various media are classified along a richness continuum. FtF communication, the benchmark against which all media are compared, is the richest form of communication while numeric documents and report summaries are the least rich (Daft, Lengel \& Trevino, 1987). Email, the media examined in this study, rests on the lower side of the richness continuum.

While MRT has been shown to be an unreliable predictor of channel selection (Markus, 1994) and the classification of email as a medium low in richness has been called into question (Carlson \& Zmud, 1999; Lee, 1994; Ngwenyama \& Lee, 1997), it has nonetheless spawned a significant body of research relating media characteristics to 
communication performance and perceptions (e.g. Carlson, George, Burgoon, Adkins \& White, 2004; Dennis \& Kinney, 1998; Dennis \& Valacich, 1999; Mennecke, Valacich \& Wheeler, 2000).

Dennis and Valacich (1999) summarized media characteristics relevant in Information Systems research into the constructs: immediacy of feedback, symbol variety, parallelism, rehearsability and reprocessability. The current study adopts these constructs to differentiate $\mathrm{CMC}$ and $\mathrm{FtF}$ communications with two alterations. First, the construct of parallelism, which considers the number of simultaneous conversations enabled by a media, is dropped as its applicability to emotional intelligence research in virtual teams is not apparent. Second, the construct immediacy of feedback is replaced with media synchronicity (Carlson et al., 2004) as the latter construct captures the ongoing nature of CMC communications as opposed to a one by one examination of individual messages and responses suggested by the former construct. Table 1 defines the remaining media characteristics and differentiates $\mathrm{FtF}$ and $\mathrm{CMC}$ communication with respect to them.

\begin{tabular}{|l|l|l|}
\hline \multicolumn{1}{|c|}{ Characteristic } & \multicolumn{1}{c|}{ CMC } & \multicolumn{1}{c|}{ FtF } \\
\hline $\begin{array}{l}\text { Synchronicity - Degree to which } \\
\text { medium supports synchronous } \\
\text { interaction. }\end{array}$ & $\begin{array}{l}\bullet \text { Asynchronous } \\
\bullet \text { Low to high time } \\
\text { lapses }\end{array}$ & $\begin{array}{l}\bullet \text { Instantaneous/Concurrent } \\
\text { Feedback }\end{array}$ \\
\hline $\begin{array}{l}\text { Symbol Variety - Number of } \\
\text { available cues and channels. }\end{array}$ & $\begin{array}{l}\bullet \text { Single channel } \\
\bullet \text { Multiple cues }\end{array}$ & $\begin{array}{l}\bullet \text { Multiple channels } \\
\bullet \text { Multiple cues }\end{array}$ \\
\hline $\begin{array}{l}\text { Rehearsability - Extent to which a } \\
\text { communicator may 'practice' } \\
\text { their message before sending. }\end{array}$ & $\begin{array}{l}\bullet \text { Situation dependant } \\
\text { Typically high }\end{array}$ & $\begin{array}{l}\bullet \text { Situation dependant } \\
\bullet \text { Typically low }\end{array}$ \\
\hline $\begin{array}{l}\text { Reprocessability - Extent to } \\
\text { which a communicator may } \\
\text { revisit or 're-consume' messages. }\end{array}$ & $\bullet$ Typically high & $\bullet$ Low \\
\hline
\end{tabular}

Table 1: A comparison between Face to Face (FtF) and Computer-Mediated Communication (CMC) characteristics. 
Synchronicity is essentially defined by $\mathrm{FtF}$ interaction. $\mathrm{FtF}$ communicators may give and receive feedback while a conversation is taking place. In contrast, CMC communicators must wait an indeterminate amount of time for the message recipient to respond. The amount of time which passes before a response is delivered is a matter of individual choice but, like other media characteristics, may also be influenced by the socially constructed norms of communication within a given context (Rice, 1993).

Symbol variety considers two continuums: number of cues and number of channels. In FtF interaction both visual and auditory channels are available to communicators. The auditory channel delivers lexical information (i.e. the words used) and some paralinguistic cues, such as voice tone and inflection, while the visual channel delivers paralinguistic cues such as facial expressions and changing body positions (Scherer, 2003). In CMC only the visual channel is available for communication and therefore, the lexical encoding of information is the primary cue available. However, some preliminary work (Boonthanom, 2004) has offered a new definition of paralanguage in email text achieved through textual effects such as capitalization and punctuation based cues such as exclamation marks and 'emoticons'. Multiple cues, then, are available to $\mathrm{CMC}$ users, dependant upon the communication context and experience with the medium.

Rehearsability is typically low in FtF interactions as the bulk of this communication occurs on an ad-hoc basis. In fact, the increased level of synchronicity appears to result in a reduction of rehearsability. In contrast, CMC users can prepare their messages in isolation from their intended recipients. This results in the opportunity to alter the message a number of times before sending it which may also be influenced by 
synchronicity norms. In this study, tailorability (Carlson et al., 2004) or the ability to construct similar messages to multiple recipients in various ways is completely subsumed within rehearsability for reasons of parsimony and the logical correlation between these constructs.

Finally, reprocessability is a characteristic of CMC which may be considered an area of richness not available in $\mathrm{FtF}$ interactions. A message sent via email can be saved for any amount of time and re-examined or 'consumed' over and over. While FtF communicators may have memories of past conversations, these memories are not the actual conversation and may consist of formed judgements and attributions as opposed to actual message content.

MRT posits that individuals who choose a medium appropriate to the conveyance of meaning based on message ambiguity will achieve more successful communication. However, other researchers have suggested that fully understanding media choice requires an understanding of numerous other factors. Markus (1987) suggests that a new medium must achieve critical mass in use for its survival. Within this view, media adoption is not merely a matter of individual choice but is also influenced through social and organizational pressures (Markus, 1994; Markus, 1987).

Lee (1994) offered that it is communication which demonstrates richness, not the media itself and therefore understanding how richness occurs should provide more practical implications. This belief has largely formed the second approach to studying information systems which has been labelled here the 'media adoption/adaptation' approach. This approach involves adoption of information systems which support the needs of the users but also considers how the users adapt their own communication to the 
medium, as well as how they adapt the medium to suit their purposes. Table 2 summarizes the major theories derived from this approach which are considered in this study.

\begin{tabular}{|l|l|}
\hline \multicolumn{1}{|c|}{ Theory } & \multicolumn{1}{c|}{ Main Position } \\
\hline $\begin{array}{l}\text { Channel Expansion Theory } \\
\text { (CET: Carlson \& Zmud, 1999) }\end{array}$ & $\begin{array}{l}\text { Richness perceptions increase with richness } \\
\text { experiences for any medium. }\end{array}$ \\
\hline $\begin{array}{l}\text { Critical Social Theory } \\
\text { (CST: Ngwenyama \& Lee (1997) }\end{array}$ & $\begin{array}{l}\text { Richness is derived not from the medium, but } \\
\text { from the extent to which message recipients are } \\
\text { able to extract valid meanings. }\end{array}$ \\
\hline $\begin{array}{l}\text { Social Identity model of } \\
\text { Deindividuation Effects } \\
\text { (SIDE: Spears \& Lea, 1994) }\end{array}$ & $\begin{array}{l}\text { CMC users form less distinct identities from } \\
\text { those with whom they communicate. }\end{array}$ \\
\hline $\begin{array}{l}\text { Social Information Processing } \\
\text { (SIP: Walther, 1992; 1995) }\end{array}$ & $\begin{array}{l}\text { CMC users employ verbal cues to the same or } \\
\text { similar effect as FtF communicators use } \\
\text { nonverbal cues. }\end{array}$ \\
\hline $\begin{array}{l}\text { Adaptive Structuration Theory } \\
\text { (AST: Poole \& DeSanctis, 1989) }\end{array}$ & $\begin{array}{l}\text { Social structures are the result of the } \\
\text { appropriation of technology within its context } \\
\text { of use. }\end{array}$ \\
\hline
\end{tabular}

Table 2: Media usage theories developed within the 'media adoption/adaptation' approach to CMC research.

Carlson and Zmud (1999) based Channel Expansion Theory (CET) on their findings that perceptions of the richness of a medium increase as users experience rich communications. Proponents of Critical Social Theory in Information Systems research (e.g., Ngwenyama \& Lee, 1997) suggest that richness in communications occurs as users create meaning from messages. Gefen and Straub (1997) found that there is a gender effect with media perceptions. Women tend to perceive email as conveying less social presence and that it is less useful and easy to use. These perceptions, however, did not influence women's level of email use.

Other theorists have concerned themselves with the study of how users employ communication cues within a medium to achieve rich communications. The Social 
Identity model of Deindividuation Effects (SIDE: Spears \& Lea, 1994) posits that the limited social cues available to CMC users have a de-individualizing effect. In this view, CMC users form a stronger group, as opposed to individual, identity. Tanis and Postmes (2003), however, found that the group effect did not seem to exist. Instead, the limitation in social cues in CMC seemed to enhance positive relationship formations between communicators.

This particular finding is consistent with Social Information Processing (SIP: Walther, 1992; 1995) which posits that limited availability of certain social cues in CMC leads to more positive impression projections and perceptions by individuals. Walther and Burgoon (1992) found strong support that relationship formation is initially slower for CMC users than for FtF communicators, but that CMC-based relationships quickly rose to positive levels. Overall, temporality had a greater effect on the relational aspect of CMC use than the medium itself. More importantly, SIP proposes that CMC users adapt their communication styles such that their messages contain as many social cues as in $\mathrm{FtF}$ interaction (Walther, Loh \& Granka, 2005). In adapting to the medium, CMC users employ verbal cues where FtF users employ non-verbal cues in developing affective relationships.

Adaptive Structuration Theory (AST: Poole \& DeSanctis, 1989) is an outlier amongst these theories in that it considers the organization as a whole as opposed to individuals or communication dyads as the unit of analysis. The central position of AST is that new organizational and social structures arise from the appropriation of information technologies and that these are influenced by task and organizational factors, in addition to technological factors (DeSanctis \& Poole, 1994). AST proponents consider 
the people who use information systems as an integral part of the system. Technology alone is therefore not predictive of how an organization structure will form. Instead, users of technology adapt it to their needs as much as it adapts the manner in which processes are performed.

AST has been applied to the study of group support systems (Gopal et al., 19923) finding that the theory adequately describes the process through which existing groups appropriate new technology systems. Majchrzak, Rice, Malhotra, King and Ba (2000) explored the applicability of AST to virtual teams finding that there is a significant tension between the 'spirit' of new technology and existing social structures. This tension arises from any misalignment between the intentions of the users and the intended use of the technology. As a result, users of the technology play a significant role in how it is eventually deployed and used.

\subsubsection{Communicative Acts}

CMC communication is a skill which differs from FtF communication due to the constraints of the communication medium. Before assessing the effectiveness of a CMC user's communication, one must understand the goal of the communicator. Te'eni (2001) proposes a model of organizational communication inclusive of CMC characteristics, message form, goals and strategies. According to this model, the goals of communicators motivate them to choose one of a variety of communication strategies. Each of these strategies involves choosing a medium and constructing the form of the message, including language use, affective encoding and formality. It is worthy of note that the 
effectiveness of an individual's communication may be conveniently assessed by observing whether or not their goal was met.

This study seeks to determine the extent to which one's abilities, derived from EI, partially influence the content of their messages. The categories of communicative goals considered in this study are presented in table 3 followed by a discussion on how each is specifically defined.

\begin{tabular}{|l|l|}
\hline \multicolumn{1}{|c|}{ Goal } & \multicolumn{1}{c|}{ Examples } \\
\hline Instructing Action & $\begin{array}{l}\text { } \text { Request for information. } \\
\text { •Request to perform action. }\end{array}$ \\
\hline $\begin{array}{l}\text { Managing Interdependent Action } \\
\text { (Coordinating) }\end{array}$ & $\begin{array}{l}\bullet \text { Coordinate actions. } \\
\text { •Sequence events. }\end{array}$ \\
\hline Managing Relationships & $\bullet$ Establish relationships. \\
& $\bullet$ Maintain relationships. \\
& $\bullet$ Alter relationships. \\
& $\bullet$ Sever relationships. \\
\hline Influencing & $\bullet$ Resolve conflicts. \\
& $\bullet$ Alter beliefs. \\
& $\bullet$ Alter behaviours. \\
\hline
\end{tabular}

Table 3: Communication goals. Adapted from Te'eni (2001).

Instructing actions are unambiguous requests to perform some act. A communicator with this goal in mind assumes that the requested action will be done without further communication other than perhaps a confirmation upon completion. In a work setting, an instructive action would typically occur when the message originator assumes the requested action will be done because it is part of the recipients defined role, or because the recipient has performed the action upon request in the past.

If the recipient of the message fails to perform, this may indicate inaccurate assumptions on the part of the message sender, or that something else has changed so that 
the recipient is no longer motivated to fill such requests (i.e. a change in job description or withdrawal from assigned duties). An instructive action therefore occurs when a message sender is reasonably certain that the requested action will be performed. Recognizing that a message is instructive may require an examination of the recipient's response. If the recipient responds that they have done what was requested, gives some indication that it will be done in the future, or potentially gives reasons why it cannot be done, the message eliciting this response was likely instructive.

Managing interdependent action (coordinating) essentially refers to the coordination necessary for the successful operation of any team or work group. Software development teams, for example, require significant levels of coordination to produce complete systems from interdependently developed sub-systems. Such communications may occur to define interfaces between software components or sequence the development and testing of modules. In this study, the term 'coordinating' will be used in place of 'managing interdependent action' for simplicity.

There are essentially two conditions under which a coordinating action through communication in a work setting may be deemed to have taken place. When one employee attempts to coordinate the tasks of other employees to enhance the likelihood of successfully achieving their own task, they are coordinating. The second condition involves an employee taking on a leadership role in coordinating the activities of other employees, without committing themselves to any particular task, motivated by the desire to achieve the coherent whole. In the second condition, it is not important whether the employee has a legitimate right to assume a leadership role, but instead is determined by their intention to do so. When such communications occur in the absence of reasonable 
belief that coordinating efforts will improve overall team performance, another complex form of influencing may have occurred.

When formulating a message with the intention of forming, maintaining, or severing affective bonds with other employees, the communicative act is classified as managing relationships. Messages intent on establishing task relationships (managing interdependent actions) or managing power relationships (influencing) must not be confused with the intentions of an individual to establish and maintain affective human bonds.

There are a wide variety of communicative acts which may be classified under this category. These include congratulatory messages, discussions about non-task related subjects such as sharing of common interests, friendship maintenance, or saying goodbye to an employee who will no longer be a member of a specific work arrangement. Such actions are not limited to warm affectation. Where one employee attacks the character, knowledge, or ability of another employee with the intent of indicating factors which limit the development of warm relations, an act of managing relationships may be said to have occurred. In performing this categorization, one must assume that the intent of the message sender is to alter the existing human relationship with the message recipient(s).

Influencing is the most complex form of communicative act (Te'eni, 2001). Unlike instructing actions, the result of an attempt to influence may be highly uncertain. An individual may engage in an attempt to influence even when their certainty of being successful is low. In such instances an influencing message may be sent with the expectation that further communications will ensue to reduce differences in opinions or 
beliefs. Therefore, an initial attempt to influence is likely to be followed by continued discussions on the same topic.

In email, for example, an influencing act may begin a long message thread in which new participants to the discussion may be included with each response. The most obvious form of an influencing message would explicitly include opposing beliefs such as "I know we are all used to the current work schedule, but perhaps we should consider working a couple hours later while preparing this major deliverable". Other forms of influencing, such as political actions, may be much more difficult to detect and require a deeper understanding of existing relationships and histories between employees. In the current study, political actions were not expected given the circumstances of the team and their apparently functional interpersonal relationships.

\subsection{Emotional Intelligence}

Research into Emotional Intelligence (EI) has emerged from the Organizational Behaviour and Psychology literatures. The original definition of the construct is given by Salovey and Mayer (1990) but it is the updated 'four branch' ability model (Mayer \& Salovey, 1997) which is applied in this study. According to this definition, EI essentially refers to the abilities of individuals to recognize emotions in themselves and others and to use emotional information effectively (Mayer, Salovey \& Caruso, 2004). Mayer et al. (2004) describe EI as one of a "class of intelligences including the social, practical, and personal intelligences" (p. 197) and define it as "the capacity to reason about emotions, and of emotions to enhance thinking" (p. 197). Boyatzis, Goleman and Rhee (2000) 
define the construct as "the competencies that enable people to demonstrate intelligent use of their emotions in managing themselves and working effectively with others" (p. 343) while Bar-On (2000) prefers the term "emotional and social intelligence" (p.363).

Three EI measurement instruments have been established and each is associated with a slightly different characterization of the construct. The emotional competence inventory (ECI: Goleman, 1995) measures self and peer assessed scores for various competencies grouped into four clusters. These clusters are: self-awareness, social awareness, self-management, and social skills.

The development of the emotional quotient inventory (EQ-i: Bar-On, 1997) was motivated by a desire to identify and understand the factors leading to "effective emotional and social functioning" (Bar-On, 2000, p. 364). The overall score for this test is comprised of 10 factors measuring various abilities related to emotional and social understanding, functioning, and coping.

The Mayer, Salovey, and Caruso Emotional Intelligence Test (MSCEIT: Mayer, Salovey \& Caruso, 1999) is a performance-based test which measures individuals' abilities along four branches related to recognizing, using, understanding, and managing emotions. The test provides scores for each of the four abilities as well as an overall EI score.

Despite the many positive findings produced by EI research, which are discussed later in this review, the construct has spawned some academic debate (Spector, 2005) which will not be recreated in this review (see for review: Ashkanasy \& Daus, 2005; Conte, 2005; Daus \& Ashakanasy, 2005; Landy, 2005; Locke, 2005; Matthews, Roberts \& Zeidner, 2004; Mayer, Caruso \& Salovey, 1999; Mayer et al. 2004). This debate has 
helped to refine the EI construct and its measures. However, much of the dissonance expressed by detractors can be eliminated by distinguishing between 'pure' and 'mixed' measures of EI. To be clear, measurements of EI capture not only the emotional intelligence of individuals, but to a greater or lesser extent, also capture facets of personality and general intelligence. Hence, there is a significant overlap between EI, general intelligence, and personality.

Mixed measures of EI such as the ECI and, to a lesser extent, the EQ-i exhibit higher overlap with personality than does the MSCEIT model employed in this study. In addition, mixed measures tend to be based on self-reported EI as opposed to ability-based EI. The latter is preferred in this study as it is the actual behaviour of team members that will be observed, through an examination of their email messages, rather than a selfreported explanation of what they would do in hypothetical situations. The MSCEIT model has been chosen for this study as it is the only known ability-based measure of EI with confirmed reliability. As the MSCEIT does not exhibit high levels of overlap with personality factors, the latter will be assessed separately using the Big 5 factors which are discussed later in this review.

\subsubsection{The Mayer Salovey Caruso Emotional Intelligence Test}

The MSCEIT measures EI by combining scores for four related abilities or 'branches'. The impetus of the four branch ability model of EI is that, if EI is indeed intelligence, possession of it can be observed in individual performance of tasks requiring 
it (Mayer et al., 1999). The four abilities measured by the MSCEIT are shown in figure 1. Each of these abilities, and method of measurement, is discussed next.

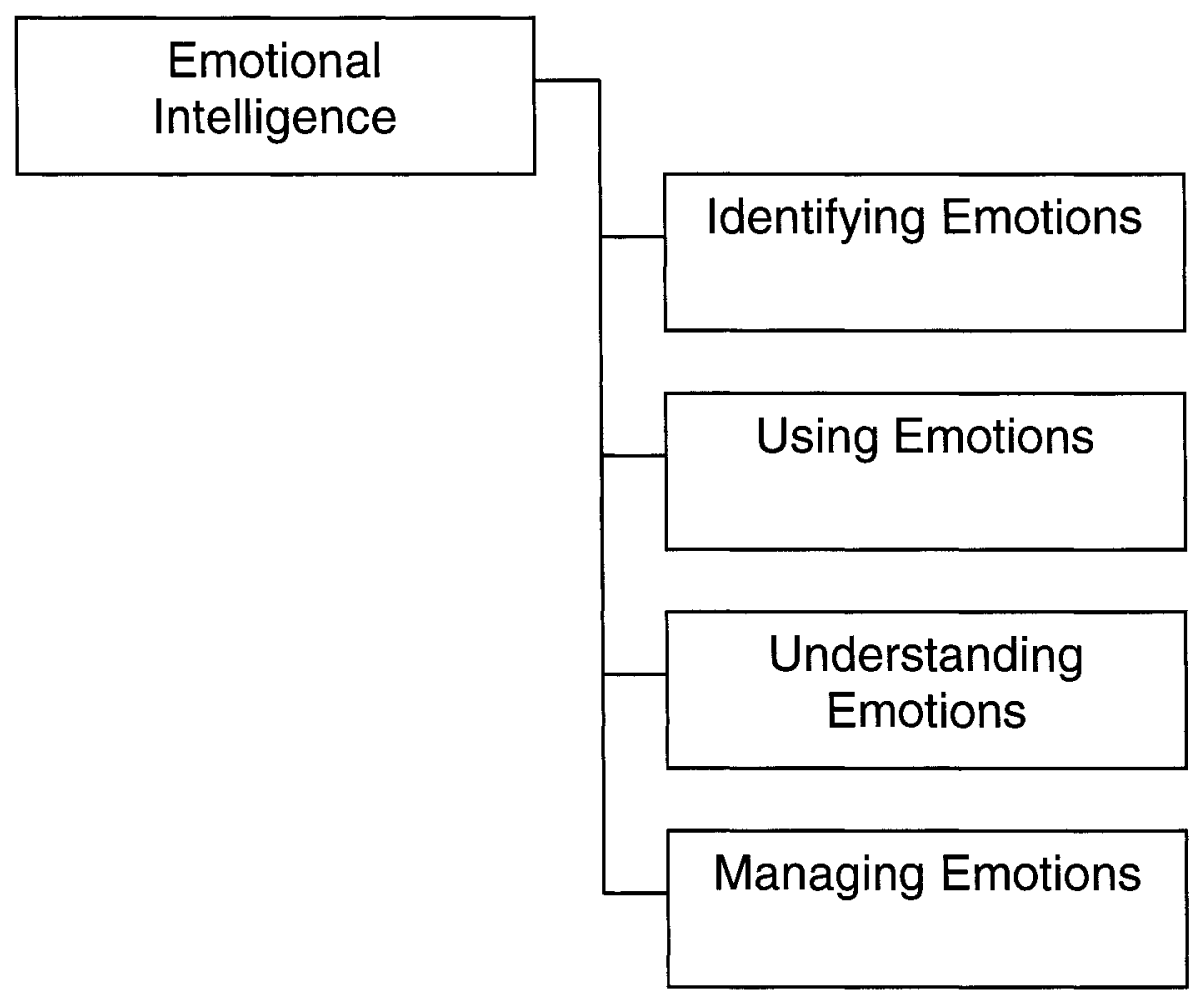

Figure 1: Four Branch Ability Model of Emotional Intelligence. Adapted from Mayer et al., 1999.

Identifying Emotions: This branch measures the ability to correctly identify nonverbal emotional expression in the face and body stance and is measured by presenting testtakers with images for which they are asked to identify the emotion depicted (Mayer et al., 2004). It should be noted here that there is no clear evidence that being able to identify emotions in facial expressions has any relationship with being able to recognize paralinguistic emotional expressions in text. This study explored this relationship to the extent possible. 
Using Emotions: This branch measures the extent to which individuals are able to use emotion to facilitate thought and is tested by asking participants to identify emotions useful in certain types of problem solving (Mayer et al., 2004).

Understanding Emotions: This branch measures the ability of respondents to predict emotional outcomes, how these change over time, and the associated outcomes of emotional episodes (Mayer et al, 2004). Test takers are asked to predict how the intensity level of emotions will change over time and to specify the composite emotions constituting more complex affective states (Mayer et al., 2004).

Managing Emotions: This branch of EI reflects the ability of an individual to manage their emotions by producing appropriate emotional displays (expressions) and suppressing inappropriate displays. This particular branch is the most related to contextual and personality factors of the four (Mayer et al., 2004). Test takers are typically asked how they would manage their own and others' emotions in hypothetical situations.

Scoring: The MSCEIT is similar in structure to a traditional intelligence test. However, the definition of the correct answer is not as straightforward as the answer to a mathematical problem. The correct answers to the MSCEIT have been determined by a panel of 'expert' scorers. These have been found to correlate highly with the more general consensus amongst test takers on the order of between 0.96 and 0.98 across the four branches (Mayer, Salovey \& Caruso, 2003). In this study, MSCEIT scores were determined in accordance with consensus scoring as these are more reliable than expert scores. 


\subsubsection{Predictive Implications of EI}

In this section of the review, findings from EI research are presented. While this literature is vast, positive findings of the MSCEIT are the focus as it is the four branch model of EI which is employed in this study. The exclusion of study results obtained using mixed measure scales is not intended to disqualify their positive findings, many of which are consistent with those obtained using the MSCEIT, but instead to focus this review. Within this section 'predictions of EI' should be read as 'predictions of EI as measured by the MEIS or MSCEIT'.

Mayer et al. (2004) summarize research findings into the categories 'academic performance' (p. 206), 'deviant behaviour' (p. 207), 'prosocial and positive behaviour' (p.207), and 'leadership and organizational behaviour' (p. 207). A final category 'development of EI and learning of emotional knowledge' (p. 209), while providing important evidence that EI follows the conventions of a standard intelligence, is not discussed here due to its inaccessibility in the current study. An assessment of ability development requires temporal information not provided in the current dataset.

\section{Academic Performance}

Lam and Kirby (2002) found that EI contributed significantly to cognitive performance beyond that accounted for by general intelligence. In unrelated studies Barchard (2003) and Brackett and Mayer (2003) found that high EI predicted academic success, a finding which is confirmed by Ashkanasy and Dasborough (2003). 
Given that the presence of strong emotions has been shown to reduce cognitive ability (e.g. Jones \& Bodkter, 2001; Von Glinow, Shapiro \& Brett, 2004), the ability to manage one's own emotions may be a key reason for the academic success effect.

Lyons and Schneider (2005) found that the emotional understanding branch of EI predicted performance on a mathematical task and that emotional management ability hampered the performance of males presenting a speech on an emotional subject. Other results indicate that the ability to manage emotions comes with certain psychological costs. Daus and Ashkanasy (2005) note that the ability to manage emotions correlates significantly with increased levels of emotional labour. There is plenty of logic in this finding. In an organizational setting, for example, one may be particularly adept at solving complex computer related issues but this does not mean they enjoy doing so.

The connection between EI and emotional labour is not isolated to the emotion management branch but extends also to the emotional recognition branch. Elfenbein and Ambady (2002) note that, in FtF interaction, high emotional recognition ability also increases self-reported measures of emotional labour in organizations. It would be inappropriate to generalize these findings beyond the academic setting in which they occur. Nonetheless, the positive findings of these studies should at least suggest the possibility that high EI individuals will also perform better in work settings, especially virtual teams, where higher cognitive ability is an asset.

\section{Deviant Behaviour}

Trinidad and Johnson (2002) found that high EI adolescents are slightly less likely to engage in self destructive pursuits such as the consumption of tobacco and alcohol. 
Brackett, Mayer and Warner (2004) found that this effect impacted males much more than females, of college age, and extended its impact to the formation and maintenance of positive relations with others. In general, Brackett et al. (2004) found males to be much lower in EI than females in the same age group and also to be more self-destructive and lower in their ability to establish and maintain positive relations with others.

There are many more common examples of deviant behaviour within organizational settings such as work-avoidance, disparaging other employees for political gain, favouring self-servitude over cooperation, fraud, and even sabotage. These activities are not only self-destructive, but can destroy organizations as well. This particular area of study examines the detrimental effects of low EI while the majority of the field extols the advantages of high EI. As this study advocates a balanced approach, and considers EI an ability which can be developed, it is possible that the selection of high EI employees may be complemented by providing EI enhancement training.

\section{Prosocial and Positive Behaviour}

Lopes, Salovey, Cote and Beers (2005) found that the emotion regulation branch of EI was positively related to the quality of social interaction and that these effects were still present after controlling for Big 5 personality traits as well as verbal and fluid intelligences. Lopes et al. (2004) found that the managing emotions branch predicted self and peer assessed levels of friendship quality with increments over the Big 5 personality traits. Lopes, Salovey and Strauss (2003) found that EI predicted self-reported satisfaction with social relationships over and above Big 5 personality measures and verbal intelligence. 
Day and Caroll (2004) found that women scored significantly higher on EI than men. In the same study an interesting finding was that EI did not predict individual organizational citizenship behaviour but did predict positive perceptions of the entire group's 'civic virtue' (p. 1456). Given that this study was experimental in nature, one should not generalize to what might be observed in an actual organization that has existed for some time.

Kafetsios (2004) found a significant positive relationship between individuals forming secure attachment styles (i.e. positive view of the self and others) and three of the four branches of EI: using, understanding and management) The ability to form positive relations with others is an important skill for anyone who works with others, which is most people, so there is little reason not to generalize these findings to the organizational context. The ability to form and promote positive social relationships within an organization is desirable in promoting cooperative work environments where conflicts are resolved effectively.

\section{Leadership and Organizational Behaviour}

The MSCEIT has been used less frequently than self-report instruments in assessing the linkage between EI and leadership. One of the few studies (Rosete \& Ciarrochi, 2005) employing the MSCEIT found that the abilities to perceive and understand emotions are significant predictors of leadership effectiveness beyond personality and other intelligence measures. Another study (Gohm, Corser \& Dalsky, 2005) found that EI predicted lower levels of stress and better ability to cope for some, but not all individuals, as the relationship was moderated by other personality traits. 
Other findings, not related to the MSCEIT, include the proposition that high EI individuals may find more constructive, rather than aggressive, behaviours when perceiving injustices in the workplace (Quebbeman \& Rozell, 2002). Also found was that EI is positively related to collaborative conflict management styles (Jordan and Troth, 2002), that EI predicted higher leadership competencies (Higgs \& Aitken, 2003) and EI was positively related to the transformational leadership style (Mandell \& Pherwani, 2003). A finding of particular importance to virtual teams is that certain aspects of EI predict the emergent leader of a self managed work team (Wolff, Pescosolido \& Druskat, 2002). In virtual teams, formal leaders are often not declared but instead emerge from the membership (Yoo \& Alavi, 2004). It seems, therefore, that EI may be a critical factor in determining which employee(s) will be deemed leaders of virtual teams.

\section{$\underline{\text { Summary }}$}

The above discussion suggests that the high EI individual is a valuable asset for modern organizations. The high EI individual is less likely to engage in self destructive behaviour, has a more positive outlook on life and others, is liked by others, may be more likely to emerge as the leader of a team, and may in fact be a better leader all around. In addition, the high EI individual may possess a better ability to harness their emotional processing savvy to complement their cognitive processing. Characterized as ability, EI may be developed over life whereas other personality traits, which sometimes predict similar behaviours, are relatively stable. 


\subsubsection{Emotional Intelligence and the 'Big 5'}

Mixed measures of EI assess certain personality traits and cognitive abilities in addition to emotional intelligence. These factors are important in attaining a better overall characterization of test subjects. As the MSCEIT exhibits lower overlap with personality, consultants typically gain further insight using an assessment of the Big 5 personality traits: neuroticism, extraversion, openness, agreeableness and conscientiousness (Caruso \& Wolfe, 2001). This study employs a similar strategy for two reasons. The first is that there is a moderate overlap between predictions based on EI and those based on certain Big 5 traits. EI researchers must therefore exercise caution when examining their results to determine the most likely causal factors. The second reason is that, while researchers in the field typically observe that EI accounts for effects above and beyond personality alone, the interaction between personality and EI in contexts is somewhat underrepresented in the current literatures.

McCrae and Costa (1987) provide a concise overview of the Big 5 traits upon which the following characterization is based. Neuroticism is essentially characterised by high levels of anxiety. Neurotic individuals tend to experience strong negative emotions more readily than their emotionally stable counterparts. It is logical to assume then that neuroticism would correlate negatively with the ability to manage emotions. As discussed later, however, this association is weak when EI is assessed using the MSCEIT.

An extraverted individual is one who is less self-conscious than their introverted counterparts. An extraverted individual is more likely to initiate conversations and develop more positive relationships with others. The relationship between this trait and 
EI is not clear. Given the pro-social behaviours of extraverts and high EI individuals it seems that one might predict the other. This effect is seen clearly in many self-reported measures of EI yet all but disappears when the MSCEIT is employed.

Individuals who are more open to experience will typically be the first to try new things. They exhibit less trepidation than their closed counterparts when confronted with unknown outcomes. In addition to being less wary of trying new things, open individuals may be more open to emotional experiences. This trait, therefore, may give its possessors greater opportunities to develop their understanding of emotions. This logical correlation is confirmed, to a moderate extent, in the literature reviewed later in this discussion.

Agreeableness may best be described as the willingness and active attempt to find common ground with others. An agreeable individual is more likely to consider the views of others and perhaps more readily adjust the expressions of their own views in social interactions. While many may find interactions with disagreeable individuals unsatisfying and uncomfortable, extreme agreeableness may also be somewhat pathological (McCrae \& Costa, 1987). An extremely agreeable individual may be compelled to achieve consensus even at the expense of their own desires and knowledge. Conversely, an extremely disagreeable person may be compelled to continue arguing their point despite clear evidence to the contrary. Again it is tempting to say that agreeable individuals may be more emotionally intelligent, and indeed the significant correlation with MSCEIT is demonstrated in the following literature review. This correlation, however, is moderate.

The final of the Big 5, conscientiousness, refers to the extent to which individuals focus on their goals and expend effort in achieving them. One might expect that highly conscientious individuals will do better work but this may be more of a by-product of the 
observed thoroughness which such individuals apply. To a less conscientious individual, the effort of their counterparts may seem overly time consuming and wasteful as, on the continuum between thoroughness and timeliness, the conscientious individual may be more inclined to pursue the former. The logical connection between this trait and EI is not clear, but is significant when compared with MSCEIT scores. Perhaps the conscientious individual is more aware of their own and others' emotions given their nature to assess multiple explanations for every problem in the course of being thorough. Perhaps this awareness also enhances their understanding of, and ability to manage, emotions. Again this correlation is moderate.

Each of the four EI branches is examined next individually highlighting both observed and logical correlations with Big 5 factors. The second task of providing logical interactions should be largely influenced by context dependent variables. Hence, an exploratory evaluation of the study's data would be prudent before offering advice to researchers in the field. To address the less complex task of identifying observed significant correlations between EI and Big 5 personality traits, a meta-analysis of current findings was conducted. To clarify these results, studies using mixed, self-report, and indirect correlations are reported in table 4 while studies employing the MSCEIT are presented in table 5. In each of these tables, a positive non-zero order correlation is shown simply as an ' $\mathrm{x}$ ' to avoid confusion caused by varying degrees of interrelatedness amongst variables. MSCEIT results show only low to moderate correlations on the order of 0.10 to 0.30 , while mixed measures additionally produce much higher correlations. 


\begin{tabular}{|c|c|c|c|c|c|}
\hline & $\mathrm{N}$ & $E$ & $\mathrm{O}$ & $\mathrm{A}$ & $\mathrm{C}$ \\
\hline Barchard (2003) & & & $\mathrm{X}$ & $\mathrm{X}$ & $\mathrm{x}$ \\
\hline $\begin{array}{l}\text { Barchard \& Hakistian } \\
(2004)\end{array}$ & $\mathrm{x}$ & $\mathrm{x}$ & $\mathrm{x}$ & & \\
\hline De Raad (2005) & $\mathrm{x}$ & & & & \\
\hline $\begin{array}{l}\text { Engelberg \& Sjoberg } \\
\text { (2004) }\end{array}$ & $\mathrm{x}$ & & & & \\
\hline Furnham (2005) & $\mathrm{x}$ & $\mathrm{x}$ & & & \\
\hline $\begin{array}{l}\text { Law, Wong \& Song } \\
(2004)\end{array}$ & $\mathrm{x}$ & & & & $\mathrm{x}$ \\
\hline Schutte et al. (1998) & & & $\mathrm{x}$ & & \\
\hline $\begin{array}{l}\text { Vakola, Tsaousis \& } \\
\text { Nikalaou (2004) }\end{array}$ & & $\mathrm{x}$ & $\mathrm{x}$ & $\mathrm{x}$ & $\bar{x}$ \\
\hline $\begin{array}{l}\text { Van Der Zee, Thijs \& } \\
\text { Schakel (2002) }\end{array}$ & $\mathrm{x}$ & $\mathrm{X}$ & $\mathrm{x}$ & $\mathrm{X}$ & $\mathrm{x}$ \\
\hline Totals (of 9) & 6 & 4 & 5 & 3 & 4 \\
\hline
\end{tabular}

Table 4: Meta analysis of current research findings with respect to the correlation between mixed and self report measures of $\mathrm{EI}$ and the Big 5 personality traits. $\mathrm{N}=$ =neuroticism, $\mathrm{E}=$ =xtraversion, $\mathrm{O}=$ openness, $\mathrm{A}$ =agreeableness, $\mathrm{C}=$ conscientiousness.

At a glance, mixed and self-reported measures of EI produce no discernable pattern of correlation with Big 5 personality traits. This is not altogether surprising as different mixed measures assess different personality traits, in addition to EI, to varying extents. Before moving on to meta-analysis results which pertain exclusively to MSCEIT and Big 5 correlations, there are two implications of the current results deserving of further discussion.

It should be acknowledged that the results of Barchard (2003) were derived from the MSCEIT scale as opposed to a mixed-measure scale. They are nonetheless included here as they do not directly assess the correlation between EI and the Big 5 traits. What is interesting, however, is that the traits openness, agreeableness and conscientiousness predicted academic success to a similar extent as EI. While this does not positively indicate that these traits and EI are significantly correlated, it is interesting to note that 
both predict the same effect and, according to Mayer et al. (2004), these predictors are correlated.

The other studies listed here employ various self-reported measures of EI. The salient difference between self-reported and ability assessment of EI is that the former relies on one's knowledge of what should be done in certain circumstances while the latter assesses one's capability to perform such tasks. There is tentative evidence in these results to suggest that neurotic and extraverted individuals will rate themselves more emotionally intelligent than others. The subsequent results suggest that these selfassessments may differ from ability-based EI measures.

\begin{tabular}{|c|c|c|c|c|c|}
\hline & $\mathrm{N}$ & $E$ & $\mathrm{O}$ & $\mathrm{A}$ & $\mathrm{C}$ \\
\hline $\begin{array}{l}\text { Brackett \& Mayer } \\
(2003)\end{array}$ & & & $\mathrm{x}$ & $\mathrm{x}$ & \\
\hline Brackett et al. (2004) & & & & $\mathrm{x}$ & \\
\hline Day \& Carroll (2004) & & & $\mathbf{x}$ & $\mathrm{x}$ & \\
\hline $\begin{array}{l}\text { Livingstone \& Day } \\
\text { (2005) }\end{array}$ & & & $\mathrm{x}$ & $\mathrm{x}$ & $\mathrm{x}$ \\
\hline \multicolumn{6}{|l|}{ Lopes et al. (2004) } \\
\hline $\begin{array}{l}\text { Lopes, Salovey, Cote \& } \\
\text { Beers (2005) }\end{array}$ & & & & $\mathrm{x}$ & \\
\hline $\begin{array}{l}\text { Lopes, Salovey \& } \\
\text { Strauss (2003) }\end{array}$ & & & $\mathrm{x}$ & $\mathrm{x}$ & $\mathrm{x}$ \\
\hline Mayer et al. (2004) & & & $\mathrm{x}$ & $\mathrm{x}$ & $\mathrm{x}$ \\
\hline $\begin{array}{l}\text { Roberts, Zeidner \& } \\
\text { Mathews (2001) }\end{array}$ & $\mathrm{x}$ & $\mathrm{x}$ & $\mathrm{X}$ & $\mathrm{x}$ & $\mathrm{x}$ \\
\hline Totals (of 9) & 1 & 1 & 6 & 8 & 4 \\
\hline
\end{tabular}

Table 5: Meta analysis of current research findings with respect to the correlation between abilitybased measures of $\mathrm{EI}$ and the Big 5 personality traits. $\mathrm{N}=$ =neuroticism, $\mathrm{E}=$ extraversion, $\mathrm{O}=$ openness, $\mathrm{A}=$ agreeableness, $\mathrm{C}=$ conscientiousness.

Table 5 lists studies which report the correlation between Big 5 factors and MSCEIT assessed EI in this meta-analysis. The results of Mayer et al. (2004) may be the most reliable of those listed here, as these researchers had access to the greatest amount of data to determine specific correlations. It should be noted Mayer et al. (2004) found 
that all Big 5 factors are related to the overall MSCEIT score but that the results for neuroticism and extraversion are close to zero-order. The results of Roberts et al. are based on an earlier version of the MSCEIT, the MEIS, and are included here to demonstrate the refinement of the current instrument. From these results it appears that the high EI individual is somewhat more open to experience, agreeable and conscientious. The results shown are for correlations between Big 5 factors and total MSCEIT scores. Correlations with individual branches can be quite different, as will be discussed next.

\section{Identifying Emotions}

Mayer et al. (2004) note that there is a small correlation between the ability to identify emotions and agreeableness. Day and Carroll (2004), however, found similar magnitude correlations with openness and a small negative relationship with neuroticism. These results may indicate that, by being more open to experiencing emotions, an individual may enhance their ability to perceive emotions. Likewise, said individuals may be more agreeable given that they are better able to perceive the emotional state of others which enables them to behave in a sympathetic manner. Higher neuroticism may interfere with one's ability to accurately perceive emotions. However, this effect tends to be slight in most studies assessing the correlation.

\section{Using Emotions}

Day and Carroll (2004) found that using emotions correlated positively with openness and negatively with extraversion. These results again highlight the importance 
of being open to experience in developing EI skills. Indeed one learns through experience and, given that EI skills are not formally taught in most societies, being open to experience may be a prerequisite to developing these skills on one's own. The negative correlation with extraversion may indicate that a certain degree of introspectiveness is required to develop the ability to use emotions. Both Lopes et al. (2003) and Mayer et al. (2004) find that one's ability to use emotions is also positively related to their agreeableness. Being agreeable may therefore allow individuals to use their emotions more effectively, given that they will form more functional relationships with others.

\section{$\underline{\text { Understanding Emotions }}$}

Individuals who are better able to understand emotions tend to be more open (Mayer et al. 2004), less extraverted (Day \& Carroll, 2004) and more conscientious (Lopes et al., 2003). The correlations with extraversion and openness are likely for reasons similar to those already discussed. In addition, a conscientious person may spend more time determining causes and progressions of emotional states in themselves which in turn may help them to understanding the emotions of others.

\section{$\underline{\text { Managing Emotions }}$}

Being able to manage one's own and others' emotions has been shown to correlate with agreeableness (Lopes et al., 2004; Lopes et al., 2005; Mayer et al., 2004), openness (Day \& Carrol, 2004; Mayer et al., 2004), conscientiousness (Lopes et al., 2003; Mayer et al., 2004) and extraversion (Lopes et al., 2004; Mayer et al., 2004). It is perhaps telling that an agreeable extravert will be better at managing the emotions of 
others. Also, it is logical that being poor at managing one's own emotions actually makes one disagreeable.

\section{Summary}

Personality traits typically predict how one might behave in both social settings and on their own. The set of abilities derived from one's EI may also influence how an individual behaves but only to the extent that they actually use their abilities. Certain personality traits such as openness, agreeableness and conscientiousness may influence the development and use of EI abilities but not to an extent which suggests that EI and personality are the same constructs. EI, as represented by a set of abilities, gives an objective assessment of what individuals are capable of doing and personality traits provide a measure of what individuals are inclined to do. For practical purposes, including an examination of virtual team communication effectiveness, both must be considered for the combined effect of possessing and inclination to use EI abilities.

\section{3 Emotion, Emotional Intelligence, and (Virtual) Work}

Information Systems literature has developed a wealth of knowledge on topics which directly influence the performance and effectiveness of virtual teams. Many of these topics, such as trust, conflict and leadership have affective overtones. However, emotion is seldom at the centre of analysis in these works. The role of emotion at work, however, has received significant attention in the Organizational Behaviour literature 
with topics including anger in the workplace, emotional labour and the emergence of the 'group affect' construct.

In a review of current research in emotion and work, Ashkanasy, Hartel and Daus (2002) note that current streams include mood effects, emotional labour and emotional intelligence. In this review, affective components of work are divided into two categories. The first, display and regulation of emotions, includes the topics: mood and emotion, emotional labour, and group affect. The second category focuses on affective experiences, resulting from work processes, including job satisfaction, trust, conflict and leadership. Affective experiences are inevitable in the workplace and may result in positive or negative outcomes. The extent to which one possesses abilities derived from their EI should play a significant role between affective experience and outcome. Throughout this review the implications for EI are related to the positive findings of the studies examined.

\subsubsection{Emotions: Display and Regulation}

\section{Mood and Emotions}

Research into emotions in the workplace began in the 1930's but primarily focused on job satisfaction until the mid-1980's which began a period of serious inquiry on the subject and broadening of the scope of affective experience in the workplace (Brief and Weiss, 2002). The motivation for broadening our knowledge of affective experience at work is succinctly stated by Brief and Weiss (2002, p.280): "The organizations in which people work affect their thoughts, feelings, and actions in the 
workplace and away from it. Likewise, people's thoughts, feelings, and actions affect the organizations in which they work." The causes and consequences of emotional experience are often a product of the work environment itself (Frost, 2004) or caused by an unanticipated event (Miller, 2002). Such experiences are typically inevitable and must be managed to ensure an effective and productive workplace. However, managing emotions out of the workplace is most likely impossible (Fineman \& Sturdy, 1999).

The experience of positive emotions has been shown to enhance decision making ability and, not surprisingly, to enhance the interpersonal relationships of workers (Staw \& Barsade, 1993). Wright and Staw (1999) found that those possessing a disposition for positive affect perform better at certain tasks. Staw, Sutton and Pelled (1994) found that employees who experience more positive emotions are rewarded by their supervisors with more favourable performance reviews, social support and higher pay. From these results it may be inferred that individuals who are better able to use and manage their emotions will be more highly regarded by their employers, be considered for more promotions and form more effective interpersonal relationships with their co-workers.

It has also been proposed that experiences of positive emotions in the workplace will enhance the likelihood of employees engaging in organizational citizenship behaviours while negative affect tends to influence counterproductive behaviours in the workplace (Spector \& Fox, 2002). These and many other studies provide a strong suggestion of the power of positive emotions in the workplace. The use of CMC technology, as is common in virtual teams, may confound higher EI individuals attempting to use positive emotions effectively. The ability to manage the emotions of others in a virtual environment may, as yet, be an unidentified skill. 
Negative emotions, such as anger, have become a widely studied area. Workplace bullying resulting from high levels of conflict intensity has been found to increase levels of negative affect in both targets and observers of the bullying and this effect influences the propensity of employees to engage in counterproductive behaviours (Ayoko, Callan \& Hartel, 2003). Lee and Allan (2002) found that counterproductive behaviours extending from affective experiences are typically targeted towards other employees, suggesting that bullying begets bullying.

Anger is experienced more often by employees who deal directly with people as part of their job role but these individuals tend to express their anger less directly than managers (Sloan, 2004). Brown, Westbrook and Challagalla (2005) noted that the expression of negative emotions in response to an affective event reduces worker performance. Co-worker initiated aggression has been shown to reduce employee commitment while publicly initiated aggression increases turnover intentions (LeBlanc \& Kelloway, 2002). It has been suggested that the biggest causes of anger in the workplace stem from perceptions of unjust treatment and immoral behaviour (Fitness, 2000).

Extending this discussion to a virtual team, it is clear that aggression and other counterproductive behaviours will emerge in different forms than in a conventional workplace arrangement. Those engaging in aggressive behaviour may write disparaging emails to other employees but, in doing so, must realize that their messages may be viewed by anyone once they are sent, given the reprocessability of the medium. As a result, during the rehearsal of an email message the contents of such emails may be varied to such an extent that they are ultimately very different than the contents of a 
message delivered through speech. The challenge of identifying and managing emotions in email adds a new dimension to the EI construct.

\section{Emotional Labour}

Emotional labour was first described as the requirement to display unfelt emotions while suppressing felt emotions (Hochschild, 1983). The original conception of emotional labour was typically used to describe the work of service representatives whose job entailed acting as the interface between the company and its customers. This work requires that employees put on an appropriate emotional display, which can involve negative as well as positive emotions (Morris \& Feldman, 1996), regardless of what they may actually feel. Indeed, for some types of organizations, such as customer call centers, the job is emotional labour (Brannan, 2005; Mulholland, 2002). Mann (1999) notes however that non-frontline employees may engage in as much emotional faking and suppressing as those in frontline positions. As well, professional workers such as professors (Ogbonna \& Harris, 2004) and barristers (Harris, 2002) engage in emotional performances congruent with the expectations of their profession, as opposed to the organizational constraints faced by service workers.

Emotional labour is deemed to have a negative impact on those who must perform it for extended periods. Ashforth and Humphrey (1993) propose that being able to openly express one's feelings is directly related to well-being. Conversely, displaying unfelt emotions and suppressing felt emotions causes psychological fatigue, dissonance and even a loss of identity. Diefendorff and Gosserand (2003) propose that emotional labour can be de-motivating and lead to physical burnout. Dissonance caused by emotional 
labour has been found to be greater for men than for women, for older rather than younger workers and for those with no emotional attachment to their customers (Kruml \& Geddes, 2000). In some work settings, emotional labour causes a spread of negative affect amongst employees so that higher levels of dissonance experienced by one employee influence the emotions of others (Korczynski, 2003). Wages for jobs which are primarily challenging due to emotional labour are typically low but wages for those who are engaged in work that is both emotionally and cognitively challenging are amongst the highest (Glomb, Kammeyer-Mueller \& Rotundo, 2004).

Several strategies have been observed to provide relief for the dissonance emotional labour creates. Ashforth and Kreiner (2002) suggest that emotional dissonance may be reduced by workers through rituals to gain a sense of control, that emotional labourers may become desensitized to the emotional nature of their work over time and that workers both actively and passively re-conceptualize their work. The emotional labour performed in certain jobs has been compared to playing a role in a larger performance. Getting into 'character' for their role in a performance potentially reduces emotional dissonance for some workers (Guerrier \& Adib, 2003). Shuler and Sypher (2000) have even suggested that some individuals seek emotion work for altruistic reasons or by preference.

The extent to which emotional labour is a factor in virtual teams has yet to be fully established. It has been proposed that increased FtF interaction increases emotional labour while autonomy reduces it (Morris \& Feldman, 1996). These propositions suggest that emotional labour may be experienced less in virtual communications. Hiding behind the relative psychological invisibility and anonymity provided by $\mathrm{CMC}$, users may find it 
easier to feign emotions than in FtF interactions. However, the isolation from their peers may produce a new form of emotional labour for CMC communicators, specifically dealing with one's emotional experiences alone (Mann, Varey \& Button, 2000). Emotional labour in virtual teams, then, may require a revised definition.

It stands to reason that those better able to manage and use emotions may be better equipped to perform emotional labour. Shaubroek and Jones (2000) unsuccessfully attempted to find an emotional 'adaptability' linkage with reduced emotional dissonance in emotional labourers where adaptability was operationalized through personality traits. The abilities-based model of emotional intelligence may illuminate this effect. The ability to detect emotions, however, has been shown to create increased emotional labour workloads for individuals in non-service sector jobs (Elfenbein \& Ambady, 2002). For those possessing this ability to a greater extent, the observation of emotional cues 'leaked' by individuals causes dissonance between what the speaker is saying and what they are imperfectly suppressing. This review suggests that virtual team members may find it easier to feign and suppress emotions in their communications with other members but that they may find it more difficult to deal with their emotions in isolation. The ability to detect emotions in others where the primary communication medium is email may be a whole new measure of emotional intelligence.

\section{Group Affect}

George (1990) proposed that individuals would be attracted to groups whose members' personality traits, as measured by inclination for positive or negative affectivity, were similar to their own and further that such individuals were more likely to 
be selected by the group. As a result, groups could be characterized by an affective tone observed by within-group homogeneity of personality and behaviours (George \& James, 1993). Other research on the group affect construct has proposed that, by observing emotional reactions of group members to events which affect the group, individuals are 'trained' to display similar emotions under similar circumstances (Bartel \& Saavedra, 2000; Kelly \& Barsade, 2001). It has even been proposed that group members actually feel the emotions of others within the group and that the group mood directly influences individual moods (Spoor \& Kelly, 2004; Totterdell, Kellet, Teuchmann \& Briner, 1998).

Researchers should proceed with caution when examining these results. Emotions are individually, not collectively, felt. Observing that one individual 'catches' the mood of the group may incorrectly prescribe the proximal event which elicits the emotion. For example, if the team leader is in a very bad mood due to disappointing sales figures, other members may report similar moods. While it might be argued that other members of the team are trained to feel and display emotions congruent with their influential team leader, it can also be argued that what is bad news for the team leader is also bad news for the rest of the group. The team leader may fear losing his or her job while the employees fear working longer hours over the holidays. The valence and intensity of emotions may be similar, but the proximal causes are not equivalent. Also, it is quite possible that group members are feigning, as opposed to feeling, emotions they have learned are appropriate.

While George (1990) found some evidence that group personality influences performance, where positive affectivity is beneficial and negative affectivity is not, Neuman, Wagner and Christianson (1999) note that there is a significant effect associated with personality diversity. These researchers found that varying levels of extraversion 
and emotional stability were predictors of team performance while elevated levels of agreeableness, conscientiousness and openness were beneficial. While the observation that groups comprised of similar individual personalities may be more cohesive than other groups, it is not clear that such groups experience better performance. Mullen and Copper (1994), for example, find that performance influences cohesiveness more than cohesiveness influences performance. Therefore, regardless of the potential conflicts experienced along the way, a high performing team may become more cohesive by experiencing early and repeated successes.

In a virtual team, members are confronted with fewer opportunities to observe the emotional displays of other members, especially where members are actively suppressing their emotions. Emotional entrainment, therefore, may be nothing more than a representation of the norms which dictate what should and should not be encoded in a message. An individual who is better able to perceive and understand emotions may adapt to the communication norms of the group more readily. Someone who is 'emotionally illiterate' may consistently break such norms by encoding inappropriate emotional expressions in their email. An individual who is particularly adept at using emotions may be a key influencer of communication norms, thus setting the tone for the group mood through their own communications. Much research on the viability and predictive validity of the group affect construct is required, especially in virtual teams, where the processes of mood contagion and entrainment are constrained by medium of communication and the remote nature of the work. 


\subsubsection{Processes}

Working in a virtual team generally involves some isolation, if only from other team members. Presence in virtual teams, therefore, is psychological as opposed to physical and communication is required to establish that a person is actually 'present' in the team (Panteli, 2004). The added workload of 'establishing' presence in a virtual environment gives us some hint that geographic and temporal dispersion may cause increased workload in other areas as well. Within this section of the literature review, the areas of: trust, conflict, and leadership in virtual team research are briefly reviewed. This study contends that within each of these research areas, emotion and EI may play vital roles which have not been fully explored in the current literature.

In this section, the affective components of workplace processes are examined with implications for virtual teams and emotionally intelligent workers. This discussion begins with the historically important job satisfaction construct in which affective events theory (AET: Weiss \& Cropanzano, 1996) is presented. Also discussed is the process through which trust is established in virtual teams, the antecedents and consequences of conflict in virtual teams, and the emotional workload of leaders.

\section{$\underline{\text { Job Satisfaction }}$}

While the job satisfaction construct has gone through definitional changes over the last 80 years, the current definition: "a positive or negative evaluative judgment of one's job or job situation" (Weiss \& Cropanzano, 1996, p. 2) is now widely accepted, as evidenced in the literature reviewed here. There are two central issues for researchers in 
this field. The first is how job satisfaction is measured; either as a cognitive or affective evaluation of the job. The second is what, if anything, does job satisfaction predict. Indeed, what job satisfaction predicts is highly dependent on how it is measured. All studies reviewed here demonstrate that there are numerous factors which influence the relationship between job satisfaction and the most important end-result for organizations, namely performance. Despite this inherent complexity, job satisfaction research has yielded some of the most important results for the role of affect in the workplace to date.

Weiss and Cropanzano (1996) proposed AET to clarify the role of emotional experiences in the process of forming job satisfaction attitudes. AET posits that how employees react to events in the workplace which elicit emotional responses is the proximal cause of job satisfaction evaluations. This theory is important as it is inclusive of the role of emotions in evaluating one's job while not ruling out the influences of personality and the characteristics of the job. The general premises of AET, important in this review, are that events occur which lead to emotional reactions, moderated by the predispositions of personality traits, and that these reactions lead directly to the formation of judgments regarding one's satisfaction with their job. These effects influence judgments over and above the cognitive evaluation of job characteristics such as pay, working hours, and the relative status of a profession within the applicable society.

This theory attempts to address the non-intuitive result that employees who cognitively evaluate the characteristics of their job as good also self-report job dissatisfaction. It also helps to explain why two individuals in exactly the same profession report widely varying levels of satisfaction. For example, those in the mostly highly esteemed profession who are also paid well are not necessarily the most satisfied 
workers. It depends on their experiences at work and how these ultimately influence their judgments.

The linkage between job satisfaction and performance is not clear. Organ and Near (1985) report that cognitive evaluations of job satisfaction appear to have little relationship with performance while affective evaluations link with pro-social and organizational citizenship behaviours. Farkus and Tetrick (1989) found that job satisfaction is linked to commitment but that the predictive direction is unclear. Wolpin, Burke and Greenglass (1991) note that, in addition to affective events in the workplace, emotional stressors in one's personal life directly influence employee evaluations of their jobs. Hence, dissatisfaction with life may directly influence job satisfaction. Also, Cote and Morgan (2002) found that the need to suppress negative emotions at work leads to decreased job satisfaction and increased intentions to quit the job.

In examining personality influences on job evaluations, Brief, Butcher and Roberson (1995) find that those predisposed to respond more actively to negative affective stimulus, that is high in negative affectivity (NA) do indeed experience less job satisfaction than their positive counterparts (high PA). Furthermore, these researchers find that moderate mood enhancers, such as 'free cookies' at work, enhance job satisfaction more for the high PA worker than the high NA worker. While these results suggest that high PA individuals are more easily induced to form positive assessments of their jobs, other studies indicate that dissatisfaction can be most dire for their performance. Duffy, Ganster and Shaw (1998) found that high PA individuals, when dissatisfied with their jobs, most actively pursue counterproductive work behaviours including work reduction and actively searching for new employment. 
As stated previously, there is a major issue in job satisfaction research concerning how satisfaction is measured. Asking an employee whether they are satisfied with their pay and other conditions is apparently much different that asking them how they feel about their work. To gain a better understanding of satisfaction and its effects it is advisable to assess both cognitive and affective evaluations as they appear to be quite independent (Fisher, 2000; Ilies \& Judge, 2004). There is little to differentiate job satisfaction in a virtual, as opposed to non-virtual, team. However, Cote and Morgan (2002) note that remote workers are much more likely to be satisfied when they perceive themselves to be self-sufficient. Managers of such workers, therefore, must be diligent in providing all the necessary resources to ensure their employees are able to fulfill their duties despite limited contact with others (Staples, Hulland, \& Higgins, 1999).

There is no apparent reason to believe that an emotionally intelligent individual will be more satisfied with their job. However, it has been noted that when unique skills derived from emotional intelligence are utilized in performing the possessors' job function, they will be more satisfied with their work (Caruso \& Wolfe, 2001). Also, those able to more accurately detect emotions in others may be able to accurately assess the causes of reduced or increased job satisfaction in others. By being able to better understand and regulate their own emotions, high EI individuals may be able to separate emotional events which are not relevant to their jobs from those that are. High EI individuals, therefore, may attribute satisfaction differently than lower EI individuals. 
Trust

Ability to establish trust is often cited as a critical precursor to effective virtual team performance (e.g. Brown, Poole \& Rodgers, 2004; Jarvenpaa, Knoll \& Leidner, 1998; Jarvenpaa \& Leidner, 1999; Paul \& McDaniel, 2004; Zolin, Hinds, Fruchter \& Levitt, 2004). DeRosa, Hantula, Kock and D'Arcy (2004) note that the lack of FtF interaction between members increases the coordination and cohesion challenges in virtual teams. These authors cite that trust is more important for virtual teams given the limited visibility of members' actions. Zolin et al. (2004) found that increased diversity of team membership tends to reduce trust in virtual teams and that this relationship is moderated by perceived trustworthiness of members. More importantly, these authors found that initial perceptions of the trustworthiness of others were persistent and predicted perceptions of members' actual follow-through on commitments. Initial trustworthiness impressions, therefore, seem quite important in virtual teams.

Managers and leaders of virtual teams also play a significant role in the management of trust in a team. Behaviour control efforts, such as specifying deadlines for each team member, draws attention to any member whose tasks are not completed on time, thereby reducing their perceived trustworthiness (Piccoli \& Ives, 2003). In addition, procedural and distributive justices influence both team members' affective state and levels of trust (De Cremer, Stinglhamber \& Eisenberger, 2005; Saunders \& Thornhill, 2003).

Panteli and Sockalingam (2005) identify three types of trust: calculus based trust, knowledge based trust and identification based trust, the latter of which is considered rare. The three types of trust occur in sequence, as the team members gain experience 
working together. Calculus based trust develops when members perceive that others are trustworthy due to co-dependence. Knowledge based trust develops as members come to understand the skills and abilities of other members and, therefore, are able to form clearer perceptions of what each member is capable of offering to the team. Identification based trust occurs only in teams with a strong group identity in which all members are considered equally trustworthy. Jarvenpaa et al. (1998) found that perceptions of members' integrity and ability are keys to the early formation of trust in virtual teams while perceptions of benevolence increase in importance over time. Paul and McDaniel (2004) found that calculus, knowledge (which they term competence), and relationalbased trust all contribute to the formation and maintenance of interpersonal relationships amongst virtual team members.

Positive affectivity has been found to play a role in the formation of trust in both virtual and non-virtual teams. Jarvenpaa and Leidner (1999), for example, found that teams which develop higher levels of trust early in their existence express higher levels of optimism and affective warmth in their communications. In addition to positive affectivity, early and predictable communication is seen as an antecedent to higher levels of trust (Coppola, Hiltz \& Rotter, 2004). Based on these and other findings, Brown et al. (2004) conclude that affiliative personality types are more effective in virtual teams while hostility is always detrimental.

Young and Daniel (2003) have called for an examination of the experience of emotion in the workplace and how it influences trust. These authors divide the internal processes which lead to trust formation into those pertaining to relationship formation and those relating to cognitive assessments. While these two processes must interact to 
form a final judgment about the trustworthiness of an individual, their division suggests that how one assesses their relationship with another may impact heavily on the ultimate level of trust attained.

The unique abilities of high EI individuals may play a significant role in establishing, maintaining, and even repairing trust in virtual teams. Such individuals may be better equipped to use emotions to improve trust in initial team-building efforts. They may also be better equipped to identify emotional expressions of other team members and interpret them as a signal of declining trust. By managing their own and others' emotions, high EI individuals may be better able maintain or repair trust in a team. However, this will likely entail a significant undertaking on their part. Perhaps, a team with many high EI individuals will also encounter reduced levels of trust as all members are better able to recognize feigned or poorly concealed emotional expressions in the messages of their counterparts.

\section{$\underline{\text { Conflict }}$}

Conflict is a process through which individuals with differing opinions, perspectives, or goals negotiate their differences. Latent conflict, the presence of differing opinions or competing interests, progresses to manifest conflict once these differences are perceived by the interested parties (Pondy, 1967). The process of conflict transitions from latent to manifest when at least one of the parties realizes that opinions or goals are at odds. A variety of conflict behaviours then ensues until some resolution is made. Conflict is sometimes classified by its object. Therefore, conflict may be associated with task, process or emotions (Panteli \& Sockalingam, 2005; Pelled, Eisenhardt \& Xin, 1999). 
While carefully managed process and task conflict are conventionally considered beneficial in contemporary organizational forms, virtual team research suggests that no type of conflict is beneficial (e.g. Cramton, 2001; Hinds \& Bailey, 2003). Temporal dispersion of global virtual teams engenders greater conflict levels due to increased coordination difficulties and differing norms and customs of members tied to their local cultures (Hinds \& Bailey, 2003; Hinds \& Mortensen, 2002; Mortensen \& Hinds, 2001). Cramton (1999) suggests that asynchronous communication leads to uneven knowledge sharing thus increasing conflict potential. The SIDE effect in email, which influences users to over-attribute from limited available cues, has been blamed for increased conflict levels and more aggressive communication styles (Cramton, 1999; Friedman \& Currall, 2003).

Emotions have a clear role in the process through which conflict arises, escalates, and is resolved. Emotional states of team members influence the process of conflict regardless of its type (Perrewe \& Zellars, 1999). Given that it is often difficult to time the receipt of a message when $\mathrm{CMC}$ is used, virtual team members have limited ability to predict how the interpretation of a message with latent or manifest conflict content will be influenced by the emotional state of the recipient. Von Glinow et al. (2004) suggest that the presence of strong emotions drains cognitive resources which in turn limits the ability of virtual team members to employ effective conflict reducing strategies and therefore, under some circumstances, it is better to avoid further conflict discussions altogether. This observation is further supported by Montoya-Weiss, Massey and Song (2001) who find that the accommodation conflict management strategy has no significant 
effect on team performance while compromising actually reduces overall team performance.

Finally, team member characteristics also play a large role in how conflict is perceived and managed. Members of a culture who strongly value honour, for example, are much more likely to become offended when they perceive conflicting opinions and goals and are more likely to extend a conflict by including other members of the relevant group (Beersma, Harinck \& Gerts, 2003). Again, the SIDE effect in email communications may exacerbate this condition. While the collaborative conflict management style leads to increased perceptions of decision making quality and satisfaction, members of highly individualistic cultures are less inclined to pursue such a strategy (Paul, Samarah, Seetharaman \& Mykytyn Jr., 2004-5).

High EI may be a very valuable asset in virtual teams where potential for conflict is elevated. Where task uncertainty is high, or team members are diverse in their cultures or talents, the ability to manage emotions may lead to more favourable conflict resolutions. The ability to understand and use emotions in reasoning may assist in averting detrimental conflict episodes. In general, the inevitability of conflict in most virtual teams provides unique insight into how emotionally intelligent individuals apply their abilities. This also implies that the emotional labour load taken on by emotionally intelligent individuals may be much higher than for those employing primarily cognitive abilities. As such, EI may be an under-valued characteristic of virtual workers. 


\section{Leadership}

Leadership has been extensively examined in academic literature. It has been noted that emotions play a central role in the leadership process (Drodge \& Murphy, 2002). Consistent with the nature of the current study, this review focuses on these emotional roles. In particular, the transformational leadership style (Bass, 1985) and the role of leader and follower EI are discussed. In this discussion, the role of emotional expression and suppression are examined as they relate to focusing, motivating, and inspiring employees in a virtual team.

A transformational leader is typically charismatic and transcends self-interest for the benefit of their team or organization (Bass, 1985). In doing so, the transformational leader inspires followers to transcend their own self-interests in pursuit of the team's goals. Such leaders increase the optimism of their followers and the performance of the team (Hoyt \& Blascovich, 2003; McColl-Kennedy \& Anderson, 2002). The transformational style is most effective when the leader has the ability to recognize emotions (James \& Arroba, 2005) and is agreeable, extraverted, and high in positive trait affectivity (Rubin, Munz \& Bommer, 2005), all of which are characteristic of an emotionally intelligent individual. Effective leaders may inspire either positive or negative emotions in their followers, depending upon the situation (Michie \& Gooty, 2005; Pescosolido, 2002). The transformational style can be 'faked' by individuals who attempt to create a vision amongst employees which they themselves do not share. An emotionally intelligent leader may be better equipped to perform this acting, but emotionally intelligent followers are also better equipped to detect such insincerity (Dasborough \& Ashkanasy, 2002). 
Leading a virtual team is difficult given the absence of FtF interaction (Kirkman, Rosen, Gibson, Tesluk \& McPherson, 2002). The role becomes even more difficult when the leader is faced with the various cultural norms of a multicultural team (Pauleen, 2002). It has been noted that the most effective virtual team leaders exhibit empathy towards members (Kayworth \& Leidner, 2001-2) and that relationship-building is a central task in establishing trust and motivating virtual team members (Pauleen, 2003-4). It has also been found that leaders tend to emerge from the ranks of a virtual team (Yoo \& Alavi, 2004) and that the ability to manage group emotions is a key factor in determining which member(s) will emerge to fill the leadership role (Pescosolido, 2002). Managing group emotions is a difficult task given that team member's emotions are often derived from the leaders' actions (Mignonac \& Herrbach, 2004). Leaders must therefore be aware of how their actions influence followers and must be willing to deal with 'ugly' emotions, such as jealousy and envy, (Stein, 2005) in addition to providing appropriate, context-dependent emotional displays. The high EI individual may emerge to fill the role of virtual team leader, but must also be willing to deal with a significant level of emotional labour. 


\section{CHAPTER 3 - RESEARCH FRAMEWORK}

The avenues of research on EI in virtual teams provide valuable practical implications for management. Abilities derived from EI, which are innately possessed by certain individuals and are enhanced through use and training, should contribute significantly to the performance of such work structures. Before researchers can fully explore the many potential implications for virtual teams, as outlined in the preceding review, an assessment of the extent to which abilities derived from EI may be used by virtual workers is required. The specific goal of this study is to provide evidence to aid in understanding the extent to which these abilities are used by virtual workers in composing a message. Specifically, this study is motivated to determine the extent to which EI abilities influence email message content. In taking this approach, this study focuses on the interpersonal effects of EI which are manifested in the email communication of the team.

Determining the extent to which EI influences email message content is a crucial first step for researchers of EI effects in virtual teams and CMC usage in general as the creation of email messages is the most observable behaviour for every member in the team. In making this determination, researchers will be enabled to investigate many areas not fully addressed in the current academic literature. These areas include the emotional labour of virtual workers, the influences of mood and discrete emotions on virtual communications and the role of EI in emergent transformational leadership in virtual teams. Other areas include the role of EI in conflict escalation and resolution in virtual 
teams, the interaction of EI with virtual workers' job satisfaction and the ability of high EI virtual team members in establishing, maintaining and repairing trust.

The first phase of this study involves developing theory derived from a content analysis of email data regarding the influence of EI on email message content. In place of an a-priori theory, a research framework (figure 2) has been developed to guide the content analysis of email messages. This step is necessary to ensure that the content analysis remains focused on the goals of the study, as opposed to the many other possible interpretations of email text (Krippendorff, 2004). It must be stressed that this framework is not intent on illustrating causal factors. Instead, the various factors presented are deemed to interact in the process of composing an email message.

\section{Communicator}

\begin{tabular}{|l|}
\hline Emotional Intelligence \\
\hline Perceiving \\
Understanding \\
Using \\
Managing \\
\hline Personality \\
\hline Neuroticism \\
Extraversion \\
Openness \\
Agreeableness \\
Conscientiousness \\
\hline Cognition \\
\hline General Intelligence \\
Knowledge, Skills, Abilities \\
\hline Communicative Goal \\
\hline Instruction \\
Coordination \\
Relationship Mgmt. \\
Exert Influence \\
\hline
\end{tabular}

Social Norms Communication Norms Communicator Relationships

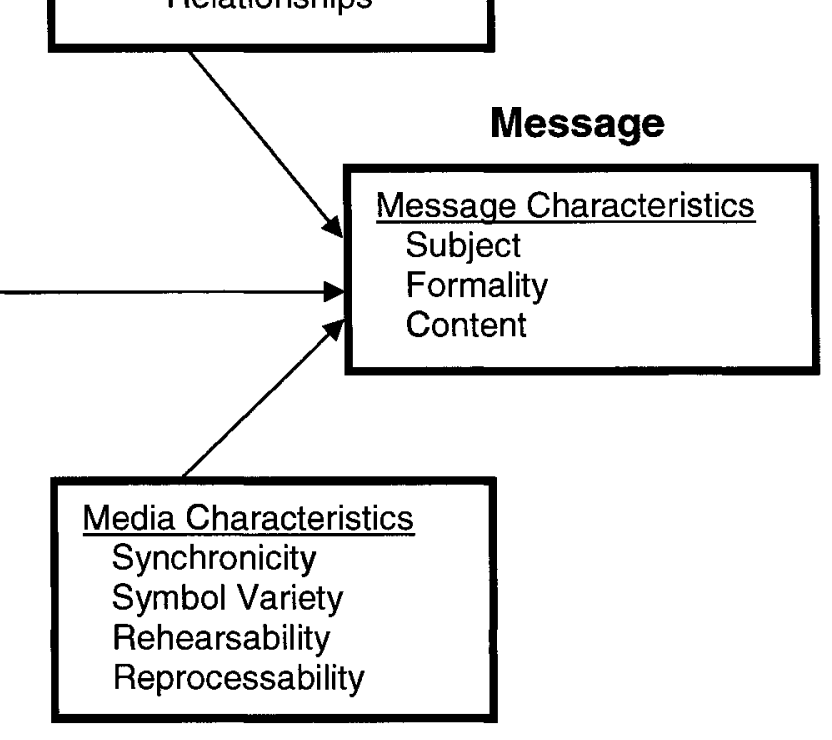

Figure 2: Factors Involved in the Process of Creating Email Messages. 
Within this framework, factors are delineated into characteristics of the communicator, characteristics of the message created and medium used as well as influences of the social network in which communications occur. Here, communicators are those composing messages through an unknown process ultimately leading to the creation of email messages. This process is deemed to be influenced by the various characteristics of the communicator as well as team and interpersonal communication norms.

The emotional state of the communicator has been omitted because, while its influence may play a significant role in how a message is created, this information cannot be reliably observed in email content produced in the past. At best, affective information can be inferred from the content of the message, while assessing affective states of the message sender would lead to very tenuous propositions. Therefore, emotional encoding will be considered for its socially constructed function, as opposed to the psychological influence on message formation (e.g. Armon-Jones, 1986). Indeed, expression or omission of emotional expressions may provide clues about the extent to which a communicator is using their EI abilities.

Communication norms here refer to the unwritten rules of the team which members apply in forming messages. The influence of communicator relationships, on the other hand, refers to certain established norms of communication which exist between individual members, where these may differ from the more general norms of the team. The analysis of these factors, in conjunction with EI, general intelligence (G) and Big 5 traits is the subject of the next section. The various analyses required in assessing message characteristics and communicative goals are partially the subject of the 
subsequent section. The email medium has already been characterized in the review, however these factors must be considered in performing the assessments of message goal and content characteristics.

In summary, this framework provides guidance for the various analyses to be conducted in this study. The results of this study were expected to partially clarify the process of email message creation by assessing the influences of EI and Big 5 traits of communicators on message content. As the relative importance of each variable presented is unclear in this process, this framework was refined in accordance with the study's findings. 


\section{CHAPTER 4 - RESEARCH DESIGN}

The case study approach is employed here as the process to be observed, that is the communication of virtual team members, is highly dependent on several contextual factors and therefore is best observed as it occurs naturally (Yin, 1994). These factors include the nature of the work performed by the group, the methods and communication norms which have developed over the course of the members' tenure, and the members' personalities and EI. Simply stated, the content of the messages produced by a virtual team will be influenced by not only objective factors, but also by contextual factors, which may not exhibit between-group consistencies.

For an exploratory study such as this, construct validity, external validity and reliability are of importance (Yin, 1994). To address construct validity, multiple sources of data will be collected. The external validity of the study, that is the domain to which the results will be applicable, was assessed and reported during the data analysis phase. At the outset, observed effects are applicable to the virtual team studied. However, great effort was taken to provide numerous propositions to enhance the generalizability of results which will form the basis of future research efforts. The subject of reliability is assessed in the context of data collection, and is discussed in a subsequent section.

\section{$\underline{4.1 \text { Participants }}$}

This study is based on a five member virtual team involved in the development and support of a proprietary information system. All five members of the team live and 
work in different areas in the Eastern United States and communicate primarily using email. The team is not part of a larger organization and relies on public funding from state and federal governments. All members are male and Caucasian. During the timeframe of the email analyzed in this study, the team had one formal leader. All five members operate from within the same time zone, although some members may sometimes travel to various regions throughout the U.S. in fulfilling their roles. Hence, temporal dispersion is not viewed as a major source of coordination challenges for the team.

\subsection{Data}

The email archives were obtained from three of the five team members. By observing the messages received by these three members it was possible to observe all messages sent by the other two members of the team with the exception of messages sent directly between the two members from whom email data was not collected. Given what is known about the communication habits of the team, it should be noted that messages from a single sender to a single recipient are very rare, and perhaps non-existent. Messages sent from August 1, 2004 to September 30, 2004 were chosen as the sampling frame for this study as the archives of all members are complete for this period. Within this frame, all messages, except those sent solely to individuals who are not members of the team, were used in the subsequently described content analysis.

Each of the five members was asked to complete the MSCEIT (Mayer et al., 2003) which requires about $30-45$ minutes to complete. The MSCEIT was chosen as it 
provides the only known ability-based measure of EI with demonstrated validity and reliability. A separate score was obtained for each of the four branches of the EI model (perceiving, understanding, using and managing), as well as an overall EI Quotient (EIQ) score. The MSCEIT was administered in an online form and results for each of the four branches, and overall EI, were provided through an automated scoring mechanism provided by the publishers of the test: Multi Health Systems (MHS), Toronto, Canada. As the MSCEIT is a proprietary instrument which must be purchased from MHS, the exact questions which the informants were asked to respond to cannot be published without permission.

In addition to the EI assessment, each member was asked to complete a 44 item instrument (John \& Srivistava, 1999) to assess their Big 5 personality traits and the Wechsler Abbreviated Scale of Intelligence (WASI: Harcourt Publishing) to obtain an estimate of G. The Big 5 instrument requires about 10-15 minutes to complete and provides scores for neuroticism, extraversion, openness, agreeableness and conscientiousness. The instrument items are detailed in Appendix A. This instrument provides individual scores for each of the Big 5 traits and was be implemented as a web application accessible by all members of the virtual team. The WASI requires about 1015 minutes to complete and was also implemented as a web application. Members were instructed that sharing of responses for any instrument(s) would limit their confidentiality. For all instruments employed in this study, personal results were made available to each team member should they be interested.

Data concerning the various communication norms of the team and a characterization of the social relationships between members were collected through 
semi-structured interviews. The questions for the structured portion of these interviews are presented in Appendix B. There are three areas that these questions were designed to address. The first was to determine the extent of communications taking place which were not represented in the email data. This determined the degree of virtuality, as defined by CMC usage, as well as the extent to which the email data accurately represented the bulk of communications. The second motivation of these interviews was to uncover any existing communications norms which individual members apply when constructing email messages. The third motivation was to determine if and why differing communication styles are employed when a team member is communicating with various other members. This aided in understanding any observed differences as email messages were categorized during analyses. Each interview was conducted through recorded telephone conversations and used as a reference in the generation of categorization schemes and to clarify results.

Together, the email archives, MSCEIT, G and Big 5 scores, and the interview results constitute the full set of data utilized in this study. The content of the email archives was analyzed in the first phase of the study, independently from other measures. The MSCEIT, G and Big 5 scores were used in the second phase in which they were related to the results of the first phase. The interview results were used in both phases to provide context to the analysis and clarify results. 


\section{CHAPTER 5 - METHODOLOGY}

This exploratory case study addresses a call to integrate qualitative and quantitative methods in Information Systems research (e.g. Lee, 1991). Qualitative research allows the researcher to situate themselves in the complex social dynamic of the structure under analysis (Denzin \& Lincoln, 2000). Grounded theory (Glaser \& Strauss, 1967; Charmaz, 2000), as applied to the study of Information Systems (e.g. Orlikowski, 1993), may employ either or both of qualitative and quantitative analyses. Indeed, it has been suggested that the Information Systems discipline should not be confined to a single over-arching research paradigm, given that such research spans the boundaries between information, technology and people in organizations (Orlikowski \& Baroudi, 1991).

To address the call to integrate qualitative and quantitative analyses, this study was conducted in two phases. The first phase involved building theory from qualitative data through a content analysis of email messages. The second phase involved deriving and testing specific hypotheses congruent with the theory generated in the first phase. These two phases are discussed next.

\subsection{Phase I: Content Analysis and Theory Generation}

This phase of the analysis was primarily concerned with content analysis of the email archives to derive a grounded theory or theories of EI influences on email message content. Each unit of analysis, as described below, was categorized according to two different schemes. The first categorization was by communicative goal, whether that be 
instructing, coordination, relationship management or influencing. The specific objective of this categorization was a determination of the primary intent of the message sender. A second, emergent categorization scheme was developed through hermeneutic interpretation of email text, directed in part by what is known about the behaviours of emotionally intelligent individuals.

Although much is known about effects predicted by EI, these effects have never been examined where the primary communication medium is email or other text-based electronic media. It is also known, for example, that email message composers may employ verbal and paralinguistic cues in place of non-verbal cues used in FtF communication. The extent to which EI influences the content encoding of a message including such cues, however, is an unknown. Therefore, in the absence of a grand theory which links the EI and CMC research domains, grounded theory approach was employed to derive theory from data (Glaser \& Strauss, 1967). The second categorization, therefore, was derived through an examination of the data. This does not mean that all relevant theory was ignored in the generation of the categorization scheme as researchers cannot be expected to abandon all knowledge in pursuit of new theory (Goulding, 2005). The knowledge which guided the generation of this categorization scheme was explicitly presented in figure 2 .

The emergent categorization was not intended to consist of the four branches of the MSCEIT EI model. There are two primary reasons why this categorization scheme was not deemed appropriate. The first is that multiple EI abilities may be employed in composing a single email message. More importantly, this study attempted to locate observable influences of EI on email message content as opposed to attempting to 
evaluate the EI of an individual by reading their messages. EI scores are best assessed through a reliable measurement instrument such as the MSCEIT and these scores were available in the second analysis phase.

Deriving a reliable and valid categorization scheme which addresses the influence of EI abilities on message content supports the objective of this study, which is to uncover the potential influences of EI abilities in email communication. Although this categorization scheme is not the primary deliverable of this study, it was intended to be an additional contribution to current knowledge. The sequence of events undertaken in this phase of the analysis and their ordering is presented in table 6 . The primary deliverable of this phase was theory of EI, G and Big 5 influences on email message content.

\begin{tabular}{|l|l|}
\hline \multicolumn{1}{|c|}{ Event } & \multicolumn{1}{c|}{ Description } \\
\hline $\begin{array}{l}\text { 1. Define communicative goal } \\
\text { categorization scheme }\end{array}$ & $\begin{array}{l}\text { Through analysis of email messages, a decision tree } \\
\text { was developed to guide raters in the categorization } \\
\text { of email messages by communicative goal. }\end{array}$ \\
\hline $\begin{array}{l}\text { 2. Derive and define EI-based } \\
\text { categorization scheme }\end{array}$ & $\begin{array}{l}\text { Through analysis of email messages, the categories } \\
\text { for the second, emergent, scheme were developed } \\
\text { and a decision tree was developed to guide raters. }\end{array}$ \\
\hline 3. Coding & $\begin{array}{l}\text { A reliability sample was categorized by two raters. } \\
\text { The research categorized all messages. }\end{array}$ \\
\hline 4. Preliminary data analysis & $\begin{array}{l}\text { Based on the coding results, EI, G, and Big 5 } \\
\text { scores, patterns in the data were sought using } \\
\text { multiple cross-tabulations of these data. }\end{array}$ \\
\hline 5. Theory Generation & $\begin{array}{l}\text { From salient patterns in the data, theory was } \\
\text { derived with respect to influences on email } \\
\text { message content. }\end{array}$ \\
\hline
\end{tabular}

Table 6: Sequence of events for Phase I of analysis. 


\subsection{Unit of Analysis}

According to Krippendorff (2004), an appropriate unit of analysis for content coding a conversation is each turn taken by the communicators. Email messages are similar to $\mathrm{FtF}$ conversations in that there is a clear delineation between each message sent. However, the probability that one email message may contain more than one subject, intention and opinion, may be higher than in a single turn taken in a FtF conversation. Therefore, caution must be taken in choosing an appropriate unit of analysis and should be driven by observing the data. For this study, it is important that each message fall into two categories, one of which being communicative goal, and the other the emergent EI-based categorization.

In choosing the unit of analysis, the most efficient way was to observe whether or not a single email message within the sample attempted to achieve two or more communicative goals. If no such messages exist, then the unit of analysis would be each email message. An example of a message which appears to be attempting to achieve two goals is given in the following fabrication:

Hi John,

Have you committed your code updates to the production server? I am getting some bugs when I try to compile.

BTW, I tried out those pointers you gave me about my swing. You were right! I got a lot more distance this weekend than usual.

Jack 
This message contains two distinct subjects, one of which relates to the work the team performs while the other relates to shared interests outside of work. The first two phrases appear to be an act of instruction, specifically a request to provide information. The remaining sentences appear to contribute to a conversation, held in the past, and concerning issues not relevant to the work related tasks of each team member. As such, it may best be described as an act of relationship management. If messages similar to this were present in the data set of the current study, the appropriate unit of analysis could not be each email message. Instead, the appropriate unit of analysis would be derived by categorical distinctions (Krippendorff, 2004).

On the other hand, if each message can be categorized into a single communicative act, there still remains the question of whether it will fall into a single category of the emergent, EI-focused categorization scheme. Although unknown, it was predicted that the emergent classification would be comprised of more abstract, and therefore general, categories. As such, if each email message fell into one communicative act category it most likely would fall into one of the emergent categories. Hence, the intention was to define the sampling unit as either the email message or categorical distinction, dependent upon presence or absence of communicative act multiplicity.

The choice of sampling unit was also motivated by the desire to retain common sampling units across each categorization scheme. In doing so, it was possible to provide a meaningful correlation between the two schemes. If each unit is classified as having a distinct communicative goal and EI categorization, it becomes possible to observe the interrelatedness of goals and EI ability usage. 
Finally, each unit of analysis was considered within the email message thread that it occurred. This applied to both the initial development of the emergent categorization scheme, and also during the subsequent coding. Examining each unit in relation to other units was necessary to extract information about the discussion in which the message had occurred. This aided the coders to better understand what was being said and why it was being said. This notion is central to the hermeneutic philosophy, as described below.

\subsubsection{Coding}

Consistent with Krippendorff (2004) and the hermeneutic approach, the content of a message is deemed to include its underlying meaning, in addition to the more mundane semantic units of words, phrases and sentences. Ricoeur (1981) defines hermeneutics as "the theory of the operations of understanding in their relation to the interpretations of texts" (p. 43). Boland (1991) was the first to advocate the use of hermeneutics in the field of Information Systems research. Hermeneutic techniques have been employed in characterizing virtual communities (Burnett, 2002; Burnett, Dickey, Kazmer \& Chudoba, 2003), in studying the role of communication norms in virtual teams (Ghosh, Yates \& Orlikowski, 2004), and the information system development process (Boland \& Day, 1989).

The philosophy of hermeneutics, or interpretation of text, is primarily concerned with the process of creating meaning from texts (Boland, 1991). Burnett (2002) notes that the process of creating meaning is an interpretive act performed by individual consumers of a text and therefore, numerous meanings may be derived from a single text. It has been 
suggested that the meaning extracted from a message does not belong to the message author (Boland, 1996). Rather, meanings are created by the cognitive and affective processing of individuals. This ultimately leads to the formation of judgements and attributions which both influence, and are influenced by, the society in which they are produced (Burnett, 2002; Phillips \& Brown, 1993; Phillips, Lawrence \& Hardy, 2004).

This study was not intent on uncovering the intended meaning of email messages or the meanings derived from them by recipients. Instead, it was motivated to understand the influences on the process through which meaning is created which drove the development of the categorization schemes. In this sense, the study borrows from ethnography in that it attempts to "understand how people enact and construct meaning in their daily lives” (Denzin, 1999, p.510).

As indicated previously, the first categorization of the email data was according to communicative act. This was accomplished by observing each unit and interpreting the most likely goal of the communicator. Precise guidelines for performing this classification were derived by iteratively analyzing data. A decision tree (Appendix C) was developed to aid in coding once the scheme was fully derived.

Once coding of communicative acts is complete, email contents were subjected to a re-analysis. In the second analysis, themes were identified within email text. Here again, meaning was important, but instead of searching for the goal of the communicator, the manner in which the message was coded, that is its contents, drove the inquiry. Here, the extents to which elements of written formality and conversational elements of speech were included in email messages were examined. Emotional expressions encoded or absent in text were treated as clues. This categorization was motivated by the desire to 
produce categories which were highly distinct from one another. A decision tree (Appendix D) was developed to aid in performing this categorization.

Once both categorization schemes were finalized, a sub-sample of 200 messages was coded by two independent raters to assess the reliability of the coding schemes. The two raters were blind to the objectives and nature of the study. The researcher performed both categorizations for the entire sample of emails. Big 5, G and EI scores were not analyzed prior to coding in order to avoid biases such as the potential influence on categorization of a message where the EI of the composer was known. This approach was taken to adequately distance the observer from the participants in order maintain qualitative reliability (Gans, 1999). As recommended by Neuendorf (2002), email messages were provided for coding in hard-copy form to allow 'marking up' of the text.

The end result of these analyses was a separate categorization for each unit of text according to two schemes. Various cross-tabulations were constructed at this point to aid in searching for patterns within the data. A cross tabulation including each of the two categories was first examined to seek potential relationships between categories. As the emergent categories were derived focusing on attributes of EI abilities and personality, these data suggested that certain abilities are used more in certain communicative acts. Specifically, an interaction effect was sought between communicative act and the emergent categories.

Several other comparisons were planned. A cross tabulation between each team member and communicative acts was performed to determine whether certain individuals were engaging in more of certain acts than others. A similar comparison was made between individuals and the emergent categories. Also, for each message sender, a 
comparison was planned between both sets of categories dependent upon message recipient to determine whether a particular sender was potentially engaging in more of certain communicative acts, and/or using EI abilities to differing extents with different message recipients. This comparison however was dropped for reasons explained in the subsequent section.

These comparisons were undertaken to aid in building theory about how the content varies from one communicator to another, how communication styles may differ depending on communicative act and how various communicative acts may be pursued using one or more EI abilities. The recorded interviews were used during this analysis to provide potential reasons for any observed differences, as expressed by the team members themselves. This qualitative analysis resulted in the grounded theory of EI influence on email message content from which hypotheses were generated and tested in the second phase of the study.

\subsubsection{Reliability}

Interrater reliability was assessed for each categorization scheme to ensure that the observed agreement was significantly above chance. In addition to percentage agreement, Cohen's kappa (Cohen, 1960) was used to determine the level of agreement beyond chance. The value of kappa which is considered sufficient involves a highly subjective determination. For this exploratory study, the value is provided simply to assess the reliability of the schemes amongst independent raters and to compare these to the agreement between independent raters and the researcher. 


\subsection{Phase II: Hypothesis Generation and Testing}

From the theory generated in phase I of the study, specific hypotheses were derived and tested. Depending on the results of the hypothesis testing, the theory generated in phase I was refined and further tested. For example, if an analysis of the data revealed effects that varied from the proposed theory, or if new effects were discovered, these were included in the results so as not to overlook potentially important findings which are relevant to the objectives of the study. The variables used in hypothesis generation and testing are listed in table 7 . The sequencing of events undertaken in this phase and their ordering is presented in table 8.

\begin{tabular}{|l|l|}
\hline \multicolumn{1}{|c|}{ Variable } & Measure \\
\hline Communicative Act Category & Nominal \\
\hline EI-based Category & Nominal \\
\hline MSCEIT Branch and Overall Scores & Ratio \\
\hline G Scores & Ratio \\
\hline Big 5 Scores & Ratio \\
\hline
\end{tabular}

Table 7: Variables Available for Analysis in Second Phase.

\begin{tabular}{|l|l|}
\hline \multicolumn{1}{|c|}{ Event } & \multicolumn{1}{c|}{ Description } \\
\hline 1. Hypothesis generation. & $\begin{array}{l}\text { Specific hypotheses were derived to test the theory } \\
\text { developed in Phase I. }\end{array}$ \\
\hline 2. Hypothesis testing. & Statistical testing of the hypotheses was conducted. \\
\hline 3. Theory refinement. & $\begin{array}{l}\text { Results of hypothesis testing were used to refine theory } \\
\text { generated in Phase I, if necessary, and events 1 and } 2 \\
\text { were repeated. }\end{array}$ \\
\hline 4. Result reporting. & $\begin{array}{l}\text { Results of the current study and implications for future } \\
\text { research were presented. }\end{array}$ \\
\hline
\end{tabular}

Table 8: Sequence of events for Phase II analysis. 
Given that the number of participants for this study was five, it was not possible to reliably assess the normality of the distribution for each variable within this group. Therefore, Spearman's Rho, a nonparametric measure of correlation, was calculated to provide a measure of association between variables in order to test the derived hypotheses. In addition, the magnitude of the associations were assessed using linear regression with MSCEIT branch and total scores, Big 5 scores and G as independent variables and proportions of messages in each category as dependent variables. 


\section{CHAPTER 6 - RESULTS}

In this section, the results of both phases of the study are presented. The findings from the telephone interviews which influenced the formation of categorization schemes are first discussed. Next, the categorization schemes are presented with definitions provided for each category. Following this, the results of the coder reliability assessment are presented as well as the various comparisons between categories that were performed. The theory of EI, G, and Big 5 traits influence on email message content is provided as the conclusion to the first phase of the study. Finally, the results of the second phase are presented in which the specific hypotheses derived from the grounded theory of phase I are disclosed and evaluated.

\subsection{Telephone Interviews}

From the telephone interviews it was determined that email messages comprised the bulk of communication for this team. Other forms of communication included FtF meetings which occurred four times per year, telephone calls which occurred at most twice a month, and instant messaging which was used at varying levels by different members of the team. Despite the availability of these other media, each member reported that email was the primary medium used by the team accounting for ninety percent or more of all communications.

Each member was asked whether they felt their email messages differed in terms of content or style when communicating with different members of the team. Each 
member reported that messages sent to individuals external to the team would be more formal, in terms of attention to spelling and grammar, and in terms of level of detail and background information given. Within the team, however, none of the members articulated any salient differences other than that the topic may be influenced by the specific roles of each member. For example, testing and evaluating software was primarily the responsibility of one individual while making changes to the software was the responsibility of another. While these two members would discuss technical matters related to the software, these topics were not usually discussed with the team's leader. It should also be noted that email messages sent to a single recipient were very rare. Usually, each message was sent to most or all of the team members. As a result, an assessment of email message content varying by recipient was not performed.

Each member was asked whether they checked their email messages while on vacation or otherwise away from work. One member stated that he never checked email messages while other members stated they checked and responded if they had the means to do so. While at work, each member reported that they usually checked their email messages within a few minutes of receiving them. They reported that the length of time which passed before they responded, however, varied greatly depending upon whether they deemed that the message was urgent, whether it actually required a response and their assessment of how busy they were at the time. Only one member indicated that he used the blind carbon copy (BCC) field when sending a message and did so only to copy the message to his own email address.

The team leader was asked to provide a brief history of the team and how they came to form a distributed work arrangement. He reported that the initial incarnation of 
the team included three of the current members and the other two joined later. Initially the team worked in a traditional office setting but as technology enabling them to work from home became available, they migrated away from this arrangement. The team still possesses this office space but uses it mainly for quarterly meetings. The team leader, as well as all other members of the team, reported that they preferred the distributed arrangement. Reasons for this included that working from home eliminates commuting time and that members experienced fewer distractions outside the office resulting in greater individual productivity.

\section{$\underline{6.2 \text { Communicative Act Categorization }}$}

All email messages sent by team members between August 1, 2004 and September 30, 2004 were compiled into a single electronic repository. All messages which were sent from a team member to an external entity without being sent to any member of the team were removed from the overall sample. The remaining sample of 1,269 messages was reproduced as hardcopies. Through an initial examination of the email messages, two were found to contain two distinctly different communicative acts within the same message. These two messages were divided to be coded separately thereby increasing the sample size to 1,271 units of analysis. Each message was then given a unique identifier for the purpose of coding.

A sub-sample of 200 messages was randomly drawn from the overall sample for reliability coding. The size of the sub-sample was chosen as it was observed that, under the assumption of constant proportion of agreement/disagreement for each category, only 
a marginal improvement in the precision of Cohen's kappa would be obtained with a larger sub-sample size. For example, doubling the sub-sample size to 400 messages would improve the precision of the kappa estimate by less than two percent, under the assumption given, and such an improvement is not substantively significant.

The two reliability coders were provided with a file containing the unique identifiers of the sub-sample, copies of the entire email sample, and the instructions presented in Appendix E which included the decision trees in Appendix C and Appendix D. Further communication with the reliability raters was limited to clarifications on the instructions. The researcher performed the categorization of the entire email sample. Reliability results are presented in table 9.

\begin{tabular}{|l|c|c|c|}
\hline & Agreement & K & PBAK \\
\hline $\begin{array}{l}\text { Rater 1 x } \\
\text { Rater 2 }\end{array}$ & $80.0 \%$ & 0.64 & 0.73 \\
\hline $\begin{array}{l}\text { Rater 1 x } \\
\text { Researcher }\end{array}$ & $86.5 \%$ & 0.74 & 0.82 \\
\hline $\begin{array}{l}\text { Rater 2 x } \\
\text { Researcher }\end{array}$ & $80.5 \%$ & 0.65 & 0.74 \\
\hline
\end{tabular}

Table 9: Communicative Act Rater Agreement.

Over half the messages in the reliability and overall samples were categorized as 'coordinating' by the reliability coders and researcher. It has been noted that the high prevalence of a particular category within a sample, as well as bias of a coder towards certain categories, can result in a kappa estimate which is too conservative (Sim \& Wright, 2005). To counter this effect, prevalence and bias-adjusted kappa (PBAK) was calculated. The overall reliability for this categorization scheme was fairly high between all sets of raters although less agreement was exhibited between the two reliability raters, 
and between reliability rater 2 and the researcher, than was achieved between reliability rater 1 and the researcher. All disagreements were systematically assessed by the researcher but no adjustments were made to the full sample rating which is used in this study. Given the exploratory nature of this study and the reasonable values of PBAK, the disagreements were deemed tolerable.

\section{$\underline{6.3 \text { Communication Style Categorization }}$}

Having examined the entire email sample, it was noted that communication styles of the five members were quite similar in that, without observing who the message was from directly, it was difficult to predict who had sent it. The one exception to this was Jason whose messages were less formal than those of the other team members. His messages lacked typical elements of written discourse such as capitalization where appropriate, proper grammar and spelling, punctuation, complete sentence structure, and in some cases spaces between words. The remaining team members' messages tended to exhibit elements of both written and spoken discourse. These members used punctuation to indicate questions, periods to separate phrases or sentences, capitalized the first letter in a sentence or phrase and usually capitalized the first letters of people's names. A common feature amongst all members' messages was the full capitalization of member names. In the telephone interviews it was learned that this was a norm of communication used to draw an individual's attention to a particular segment of the email.

Through iterative examination of the messages it was noted that certain members would consistently use certain words, where similar words could be inserted, and that the 
words chosen might vary according to the communicative act being performed. As a result, five categories based on different 'style' words emerged from the email under which each message could be categorized. It was also noted that the unit of analysis to which these style categories applied was similar to the unit of analysis to which the communicative act categories applied. Therefore, the unit of analysis for this scheme was the same as that of the communicative act scheme. The five emergent categories are now discussed.

Lean messages are those which contained very little content. An example of this category of message is one which includes only an internet address and nothing more. Another example is the use of email to forward an electronic file such as a document or piece of software. Lean messages are best described as those which have few or no equivalencies in spoken discourse. Email and other electronic media enable this sort of communication. It was foreseen that this category of message would be difficult to associate logically with any branch of EI, Big 5 traits, or G.

Team Building messages are those in which the relationship between members is highlighted in such a way as to foster a sense of identity for the team. While many of the messages categorized as relationship management employed this style it was defined in such as a way as to provide a distinction. Examples of messages using this style are those that assess the overall performance of the team, encourage or influence members to engage their talents to a given problem, highlight the importance of regular patterns of communication for the overall team, and assess the relationship between the team as whole and its customers, advocates and adversaries. In essence, messages employing this 
style seem to represent communication to or from a team as opposed to interdependent individuals.

Message which did not fit into the above two categories were evaluated by counting style key words. The remaining three categories were firm, tentative and hybrid. Firm messages are those that exhibit a higher level of conviction on the part of the message sender than those classified as tentative. Firm messages may include statements about what 'must be done', 'will be done', 'has been done' and so forth. Messages composed with a firm style do not appear to openly invite the opinions of others. For example, where a message sender indicates what 'should be done' it seems that offering an alternative might highlight a stronger disagreement than if the opinion was expressed as one of many potential courses of actions.

Tentative messages were characterized by the high prevalence of a different set of words and phrases such as 'maybe', 'might' and 'could be'. A full listing of the words and phrases used to distinguish firm from tentative messages is provided in the coder instructions (Appendix E). The process through which this categorization was performed consisted of making a separate count of firm and lean words and phrases. If the count was entirely or predominantly firm the message would be categorized as firm and vice versa. As a rule, a ratio of three to one or greater would always result in the categorization according to the favoured count. If the ratio of this count was exactly one to one then the message would always be classified as hybrid. Hybrid messages are those which are neither clearly firm nor clearly tentative but rather contain elements of both. There was no rule concerning the categorization of messages with word count ratios of two to one or five to two except that the lower count could not direct the classification. Instead, the 
reliability coders were instructed to choose between the hybrid category and the category favoured by the word count. Reliability results for this categorization are presented in table 10 .

\begin{tabular}{|l|c|c|c|}
\hline & Agreement & K & PBAK \\
\hline $\begin{array}{l}\text { Rater 1 x } \\
\text { Rater 2 }\end{array}$ & $52.5 \%$ & 0.38 & 0.41 \\
\hline $\begin{array}{l}\text { Rater 1 x } \\
\text { Researcher }\end{array}$ & $75.0 \%$ & 0.67 & 0.79 \\
\hline $\begin{array}{l}\text { Rater 2 x } \\
\text { Researcher }\end{array}$ & $56.5 \%$ & 0.44 & 0.46 \\
\hline
\end{tabular}

Table 10: Communication Style Rater Agreement.

As with the communicative act categorization, reliability rater 2 agreed with both reliability rater 1 and the researcher to a lesser extent than reliability rater 1 and the researcher. Again PBAK was calculated as the number of messages in some categories was much higher than others suggesting that prevalence might be an issue. As well, both reliability raters seemed somewhat biased towards the team building category. The agreement between reliability rater 1 and the researcher is adequate while agreement between reliability rater 2 and others was moderate. Pair-wise disagreements were again assessed but no changes were made to the overall categorization.

\subsection{Categorical Data Analysis and Theory Generation}

Due to the high variability in number of messages sent by each team member, the proportions within each category were calculated to allow for meaningful comparisons. Table 11 lists the proportions of each team member's emails in each of these categories. 
From these results it is clear that the bulk of communication for the team consists of coordinating actions. It is also appears that Jason performs more instructing communicative acts than the rest of the team.

\begin{tabular}{|l|r|r|r|r|r|}
\hline & \multicolumn{1}{|c|}{ Chris } & \multicolumn{1}{l|}{ Brian } & \multicolumn{1}{c|}{ Daniel } & \multicolumn{1}{l|}{ John } & \multicolumn{1}{l|}{ Jason } \\
\hline Coordinating & $74.77 \%$ & $64.85 \%$ & $68.46 \%$ & $77.63 \%$ & $62.06 \%$ \\
\hline Influencing & $4.50 \%$ & $18.32 \%$ & $13.46 \%$ & $9.21 \%$ & $6.91 \%$ \\
\hline Instructing & $9.91 \%$ & $11.88 \%$ & $11.92 \%$ & $7.89 \%$ & $21.70 \%$ \\
\hline $\begin{array}{l}\text { Relationship } \\
\text { Management }\end{array}$ & $10.81 \%$ & $4.95 \%$ & $6.15 \%$ & $5.26 \%$ & $9.32 \%$ \\
\hline & $100.00 \%$ & $100.00 \%$ & $100.00 \%$ & $100.00 \%$ & $100.00 \%$ \\
\hline
\end{tabular}

Table 11: Communicative Act Proportions for Each Team Member.

Table 11 lists the proportions of each team member's email in each of these categories. Here the firm style proportions exceed those of the tentative style but to different extents amongst communicators. Lean message proportions exhibit limited variance amongst members with the exception of Brian who uses this style to a lesser extent. The team building style exhibits a fair amount of variance across members while the hybrid style does not.

\begin{tabular}{|l|r|r|r|r|r|}
\hline & \multicolumn{1}{|c|}{ Chris } & \multicolumn{1}{l|}{ Brian } & \multicolumn{1}{c|}{ Daniel } & \multicolumn{1}{l|}{ John } & \multicolumn{1}{l|}{ Jason } \\
\hline Firm & $32.43 \%$ & $55.94 \%$ & $31.92 \%$ & $15.79 \%$ & $26.85 \%$ \\
\hline Hybrid & $7.21 \%$ & $8.91 \%$ & $9.23 \%$ & $10.53 \%$ & $7.23 \%$ \\
\hline Lean & $31.53 \%$ & $10.40 \%$ & $26.92 \%$ & $32.89 \%$ & $28.62 \%$ \\
\hline Tentative & $18.02 \%$ & $8.42 \%$ & $18.85 \%$ & $2.63 \%$ & $9.32 \%$ \\
\hline $\begin{array}{l}\text { Team } \\
\text { Building }\end{array}$ & $10.81 \%$ & $16.34 \%$ & $13.08 \%$ & $38.16 \%$ & $27.97 \%$ \\
\hline & $100.00 \%$ & $100.00 \%$ & $100.00 \%$ & $100.00 \%$ & $100.00 \%$ \\
\hline
\end{tabular}

Table 12: Communicative Style Proportions for Each Team Member. 
Given that communicative styles vary between team members, EI abilities, Big 5 traits and G may account for this variance to some extent. Observed differences in communicative act proportions may also be influenced by these factors but also by the specific role that each member fulfills within the team. For example, if a team member's actions are highly dependent upon the actions performed by others, one might expect that member to engage in a higher proportion of coordinating actions. In addition, it seems logical that members' communicative styles may vary in accordance to the communicative act performed. For example, an individual may use a predominantly firm style while performing instructing actions while employing a predominantly tentative style in influencing.

There may also be differences amongst team members with regards to style and act interactivity and the individual differences assessed in this study may account for that variance to some extent. To assess this possibility, a cross tabulation of communicative act proportions by communicative style proportions was performed for each member of the team. These data are presented in tables $13-17$.

\begin{tabular}{|l|l|r|r|r|r|l|}
\hline Chris & Firm & Hybrid & Lean & Tentative & $\begin{array}{l}\text { Team } \\
\text { Building }\end{array}$ & \\
\hline Coordinating & $36.14 \%$ & $7.23 \%$ & $42.17 \%$ & $12.05 \%$ & $2.41 \%$ & $100.00 \%$ \\
\hline Influencing & $40.00 \%$ & $20.00 \%$ & $0.00 \%$ & $40.00 \%$ & $0.00 \%$ & $100.00 \%$ \\
\hline Instructing & $27.27 \%$ & $9.09 \%$ & $0.00 \%$ & $45.45 \%$ & $18.18 \%$ & $100.00 \%$ \\
\hline $\begin{array}{l}\text { Relationship } \\
\text { Management }\end{array}$ & $8.33 \%$ & $0.00 \%$ & $0.00 \%$ & $25.00 \%$ & $66.67 \%$ & $100.00 \%$ \\
\hline
\end{tabular}

Table 13: Proportion of Message Styles within Each Communicative Act (Chris). 


\begin{tabular}{|l|l|r|r|r|r|l|}
\hline Brian & Firm & Hybrid & Lean & Tentative & $\begin{array}{l}\text { Team } \\
\text { Building }\end{array}$ & \\
\hline Coordinating & $57.25 \%$ & $9.16 \%$ & $16.03 \%$ & $4.58 \%$ & $12.98 \%$ & $100.00 \%$ \\
\hline Influencing & $51.35 \%$ & $16.22 \%$ & $0.00 \%$ & $18.92 \%$ & $13.51 \%$ & $100.00 \%$ \\
\hline Instructing & $79.17 \%$ & $0.00 \%$ & $0.00 \%$ & $16.67 \%$ & $4.17 \%$ & $100.00 \%$ \\
\hline $\begin{array}{l}\text { Relationship } \\
\text { Management }\end{array}$ & $0.00 \%$ & $0.00 \%$ & $0.00 \%$ & $0.00 \%$ & $100.00 \%$ & $100.00 \%$ \\
\hline
\end{tabular}

Table 14: Proportion of Message Styles within Each Communicative Act (Brian).

\begin{tabular}{|l|l|r|r|r|r|r|}
\hline Daniel & Firm & Hybrid & Lean & Tentative & $\begin{array}{l}\text { Team } \\
\text { Building }\end{array}$ & \\
\hline Coordinating & $31.46 \%$ & $10.67 \%$ & $35.96 \%$ & $12.92 \%$ & $8.99 \%$ & $100.00 \%$ \\
\hline Influencing & $22.86 \%$ & $11.43 \%$ & $0.00 \%$ & $57.14 \%$ & $8.57 \%$ & $100.00 \%$ \\
\hline Instructing & $54.84 \%$ & $3.23 \%$ & $19.35 \%$ & $19.35 \%$ & $3.23 \%$ & $100.00 \%$ \\
\hline $\begin{array}{l}\text { Relationship } \\
\text { Management }\end{array}$ & $12.50 \%$ & $0.00 \%$ & $0.00 \%$ & $0.00 \%$ & $87.50 \%$ & $100.00 \%$ \\
\hline
\end{tabular}

Table 15: Proportion of Message Styles within Each Communicative Act (Daniel).

\begin{tabular}{|l|r|r|r|r|r|l|}
\hline John & Firm & Hybrid & Lean & Tentative & $\begin{array}{l}\text { Team } \\
\text { Building }\end{array}$ & \\
\hline Coordinating & $6.78 \%$ & $8.47 \%$ & $42.37 \%$ & $1.69 \%$ & $40.68 \%$ & $100.00 \%$ \\
\hline Influencing & $28.57 \%$ & $42.86 \%$ & $0.00 \%$ & $14.29 \%$ & $14.29 \%$ & $100.00 \%$ \\
\hline Instructing & $100.00 \%$ & $0.00 \%$ & $0.00 \%$ & $0.00 \%$ & $0.00 \%$ & $100.00 \%$ \\
\hline $\begin{array}{l}\text { Relationship } \\
\text { Management }\end{array}$ & $0.00 \%$ & $0.00 \%$ & $0.00 \%$ & $0.00 \%$ & $100.00 \%$ & $100.00 \%$ \\
\hline
\end{tabular}

Table 16: Proportion of Message Styles within Each Communicative Act (John).

\begin{tabular}{|l|l|r|r|r|r|r|}
\hline Jason & Firm & Hybrid & Lean & Tentative & $\begin{array}{l}\text { Team } \\
\text { Building }\end{array}$ & \\
\hline Coordinating & $21.50 \%$ & $5.18 \%$ & $39.38 \%$ & $6.48 \%$ & $27.46 \%$ & $100.00 \%$ \\
\hline Influencing & $30.23 \%$ & $16.28 \%$ & $0.00 \%$ & $16.28 \%$ & $37.21 \%$ & $100.00 \%$ \\
\hline Instructing & $50.37 \%$ & $13.33 \%$ & $11.11 \%$ & $18.52 \%$ & $6.67 \%$ & $100.00 \%$ \\
\hline $\begin{array}{l}\text { Relationship } \\
\text { Management }\end{array}$ & $5.17 \%$ & $0.00 \%$ & $18.97 \%$ & $1.72 \%$ & $74.14 \%$ & $100.00 \%$ \\
\hline
\end{tabular}

Table 17: Proportion of Message Styles within Each Communicative Act (Jason).

These data suggest that there are many differences between team members with respect to the communicative style employed in certain communicative acts. John and 
Brian, for example, use a firm style to encode instructing acts to a greater extent than the rest of the team. Daniel appears to have a greater inclination to employ a tentative style when encoding influencing acts than the rest of the team with the possible exception of Chris. There are also similarities amongst these data such as the absence of any lean influencing acts and the high incidence of team building relationship management acts.

Overall, the differences between team member message styles employed in performing certain acts are more profound than observed differences in the proportions of communicative acts performed or styles employed. This suggests there is an interaction between message style and communicative act and that this effect differs amongst team members. For the purposes of this study, the extent to which individual differences in EI abilities, Big 5 traits, and $\mathrm{G}$ account for varying preferences in communication styles by communicative act was examined. In order to gain a better understanding of which individual differences account for greater variance in the interaction between style and communicative act, these differences were first assessed and this is the subject of the next section. The theory generated from an observation of this categorical data is that Individual differences in EI, Big 5 trait, and G scores account for variance in communicative style and this effect is moderated by the communicative act performed. The relationship suggested by this theory is depicted in Figure 3. 


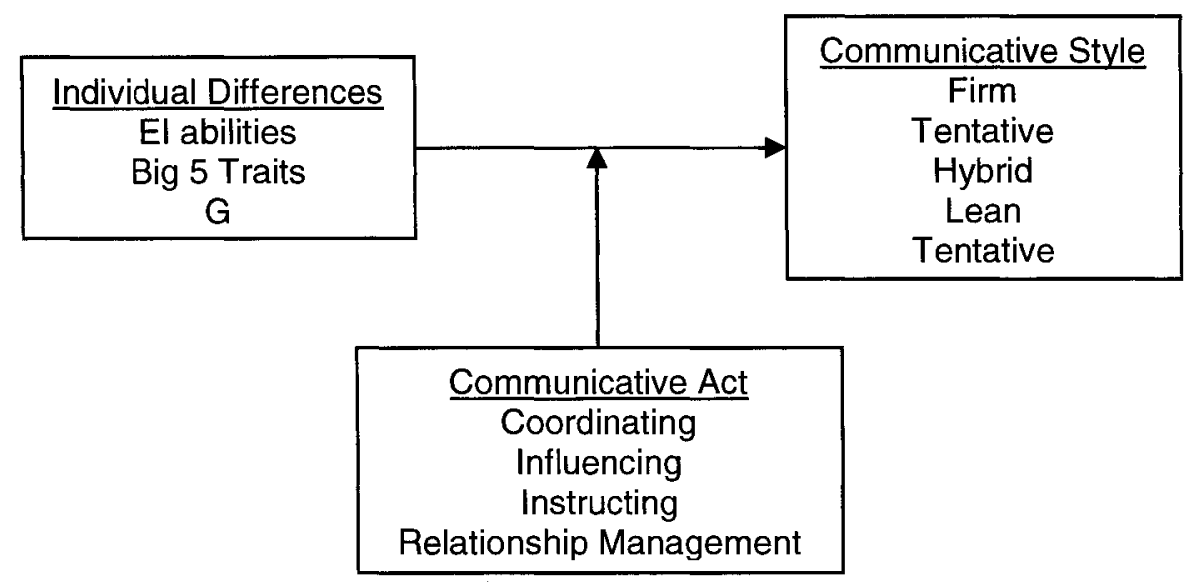

Figure 3: Model of Individual Difference Influence on Communicative Style

\section{$\underline{6.5 \text { Hypothesis Generation and Testing }}$}

Within this section, the theory derived in the preceding section is refined by examining individual differences in EI abilities, Big 5 traits and $\mathrm{G}$ and comparing these to observed differences in communicative style by communicative act. Each member's scores and qualitative assessments are first presented and apparent differences are highlighted. These differences are then compared to the communicative style differences observed in the preceding section. Finally, hypotheses are derived which are supported through this assessment and these are tested.

\section{$\underline{\text { Big } 5 \text { Traits }}$}

Trait scores for each team member are reported in table 18 with internal consistencies (Cronbach's alpha) in parentheses. Reliabilities for most of the scales are acceptable although the consistency for the conscientiousness scale is moderate. Big five 
traits were coded as $\mathrm{N}$ (neuroticism), E (extraversion), O (openness to experience), A (agreeableness) and $\mathrm{C}$ (conscientiousness).

\begin{tabular}{|l|c|c|c|c|c|}
\hline & $\begin{array}{c}\mathbf{N} \\
(\boldsymbol{\alpha}=\mathbf{0 . 9 3 3})\end{array}$ & $\begin{array}{c}\mathbf{E} \\
(\boldsymbol{\alpha}=\mathbf{0 . 8 1 2})\end{array}$ & $\begin{array}{c}\mathbf{O} \\
(\boldsymbol{\alpha}=\mathbf{0 . 9 0 0})\end{array}$ & $\begin{array}{c}\mathbf{A} \\
(\boldsymbol{\alpha}=\mathbf{0 . 6 9 8})\end{array}$ & $\begin{array}{c}\mathbf{C} \\
(\boldsymbol{\alpha}=\mathbf{0 . 5 7 5})\end{array}$ \\
\hline Jason & 3.375 & 4.250 & 4.800 & 4.333 & 3.667 \\
\hline Chris & 1.500 & 3.250 & 2.800 & 4.000 & 4.000 \\
\hline Brian & 2.750 & 2.625 & 4.700 & 3.000 & 4.778 \\
\hline Daniel & 1.750 & 2.125 & 4.100 & 4.444 & 4.111 \\
\hline John & 4.000 & 2.750 & 3.800 & 4.111 & 3.778 \\
\hline
\end{tabular}

Table 18: Big 5 Trait Scores.

Team members' scores for neuroticism exhibit substantial variance. Chris and Daniel, who scored lowest on neuroticism, both appear to use the tentative style to a greater extent and the team building style to a lesser extent than other members.

Consistent with the theory generated in the first phase of the study (figure 3), it was also hypothesized that for this and each of the following hypotheses, communicative act categories moderated the association between traits and communicative styles.

Therefore the following hypotheses were proposed:

H1: Trait neuroticism is negatively associated with proportion of tentative email messages sent, and:

H1a: This association is moderated by communicative act categories.

$\mathrm{H} 2$ : Trait neuroticism is positively associated with proportion of team building style email messages sent, and:

$\mathrm{H} 2 \mathrm{a}$ : This association is moderated by communicative act categories. 
Jason and Chris scored higher on extraversion than the rest of the team members. Fewer of their messages used the hybrid style than other members of the team leading to the following hypothesis:

H3: Trait extraversion is negatively associated with proportion of hybrid style email messages sent, and:

H3a: This association is moderated by communicative act categories.

Chris scored somewhat low on openness to experience while the remainder of the team scored moderate to high. Chris also composed the least proportion of team building style messages within the team leading to the following hypothesis:

H4: Trait openness to experience is positively associated with proportion of team building style messages sent, and:

H4a: This association is moderated by communicative act categories.

There was less variance in levels of the trait agreeableness within the team, with all members scoring moderate to high. However given that Brian scored lowest in agreeableness and a greater proportion of his messages were classified as firm, and a lower proportion of his messages were classified as lean, the following hypotheses were proposed:

H5: Trait agreeableness is negatively associated with proportion of firm style email messages sent, and:

H5a: This association is moderated by communicative act categories. H6: Trait agreeableness is positively associated with proportion of lean style email messages sent, and:

H6a: This association is moderated by communicative act categories. 
All team members scored moderate to high on trait conscientiousness. Brian, who scored the highest, produced the highest proportion of firm style messages and lowest proportion of lean style messages leading to the following hypotheses:

H7: Trait conscientiousness is positively associated with proportion of firm style email messages sent, and:

$\mathrm{H7a}$ : This association is moderated by communicative act categories.

H8: Trait conscientiousness is negatively associated with proportion of lean style email messages sent, and:

H8a: This association is moderated by communicative act categories.

Spearman's Rho and simple regressions were calculated to test each hypothesis. The results are shown in table 20 . Note that the interaction between communicative style and communicative act proportions is denoted as STYLExACT. Also, the minimum significance level was relaxed to $\mathrm{p}<0.15$ for the purposes of this exploratory study, although findings of greater significance are indicated. A legend for the variable coding is presented in table 19.

\begin{tabular}{|l|l|}
\hline T & Tentative \\
\hline TB & Team Building \\
\hline H & Hybrid \\
\hline F & Firm \\
\hline L & Lean \\
\hline C & Coordinating \\
\hline INF & Influencing \\
\hline INS & Instructing \\
\hline RM & Relationship Management \\
\hline
\end{tabular}

Table 19: Legend for Table 20 


\begin{tabular}{|c|c|c|c|c|c|c|}
\hline & Independent & Dependent & Rho & $\begin{array}{l}\text { Regression } \\
\text { Coefficient }\end{array}$ & $\mathbf{R}^{2}$ & Supported? \\
\hline H1 & $\mathrm{N}$ & $\mathrm{T}$ & $-0.90 * * *$ & $-6.429 * * *$ & 0.909 & Yes \\
\hline \multirow[t]{4}{*}{ H1a } & $\mathrm{N}$ & $\mathrm{TxC}$ & $-0.80 *$ & $-4.232 * * *$ & 0.861 & \multirow[t]{4}{*}{ Yes } \\
\hline & $\mathrm{N}$ & TxINF & $-0.90 * * *$ & $-15.177 * * *$ & 0.742 & \\
\hline & $\mathrm{N}$ & TxINS & $-0.90 * * *$ & $-12.722 * *$ & 0.684 & \\
\hline & $\mathrm{N}$ & TxRM & n.s. & n.s. & n.s. & \\
\hline H2 & $\mathrm{N}$ & TB & $1.0 * * *$ & $9.809 * * *$ & 0.909 & Yes \\
\hline \multirow[t]{4}{*}{ H2a } & $\mathrm{N}$ & TBxCOR & $1.0 * * *$ & $14.028^{* * * *}$ & 0.928 & \multirow[t]{4}{*}{ Yes } \\
\hline & $\mathrm{N}$ & TBxINF & $0.90 * * *$ & $0.9848 * * *$ & 0.888 & \\
\hline & $\mathrm{N}$ & TBxINS & n.s. & n.s. & n.s. & \\
\hline & $\mathrm{N}$ & TBxRM & n.s. & n.s. & n.s. & \\
\hline $\mathbf{H 3}$ & $\mathrm{E}$ & $\mathrm{H}$ & n.s. & $1.279^{*}$ & 0.647 & No \\
\hline \multirow[t]{4}{*}{ H3a } & $\mathrm{E}$ & HxCOR & $-1.0 * * *$ & $-2.547 * * *$ & 0.982 & \multirow[t]{4}{*}{ Yes } \\
\hline & $\mathrm{E}$ & HxINF & n.s. & n.s. & n.s. & \\
\hline & $\mathrm{E}$ & HxINS & n.s. & $6.211 * *$ & 0.719 & \\
\hline & $E$ & HxRM & \begin{tabular}{|l} 
n.s. \\
\end{tabular} & n.s. & n.s. & \\
\hline H4 & $\mathrm{O}$ & TB & n.s. & n.s. & n.s. & No \\
\hline \multirow[t]{4}{*}{ H4a } & $\mathrm{O}$ & TBxCOR & n.s. & n.s. & n.s. & \multirow[t]{4}{*}{ No } \\
\hline & $\mathrm{O}$ & TBxINF & n.s. & n.s. & n.s. & \\
\hline & $\mathrm{O}$ & TBxINS & n.s. & n.s. & n.s. & \\
\hline & $\mathrm{O}$ & TBxRM & n.s. & n.s. & n.s. & \\
\hline H5 & $\mathrm{A}$ & $\mathrm{F}$ & n.s. & $-22.547 * *$ & 0.677 & Partial \\
\hline \multirow[t]{4}{*}{ H5a } & $\mathrm{A}$ & FxCOR & n.s. & $-24.228^{*}$ & 0.556 & \multirow[t]{4}{*}{ Yes } \\
\hline & $\mathrm{A}$ & FxINF & $-0.90 * * *$ & $-18.274 * * *$ & 0.875 & \\
\hline & $\mathrm{A}$ & FxINS & n.s. & n.s. & n.s. & \\
\hline & $\mathrm{A}$ & FxRM & n.s. & n.s. & n.s. & \\
\hline H6 & $\mathrm{A}$ & $\mathrm{L}$ & n.s. & $14.870 * *$ & 0.677 & Partial \\
\hline \multirow[t]{4}{*}{ H6a } & $\mathrm{A}$ & LxCOR & \begin{tabular}{|l} 
n.s. \\
\end{tabular} & $16.454 * *$ & 0.735 & \multirow[t]{4}{*}{ Yes } \\
\hline & $\mathrm{A}$ & LxINF & n.s. & n.s. & n.s. & \\
\hline & $\mathrm{A}$ & LxINS & $0.89 * * *$ & n.s. & n.s. & \\
\hline & $\mathrm{A}$ & LxRM & n.s. & n.s. & n.s. & \\
\hline H7 & $\mathrm{C}$ & $\mathrm{F}$ & $0.90 * * *$ & $34.006^{* * *}$ & 0.882 & Yes \\
\hline \multirow[t]{4}{*}{ H7a } & $\mathrm{C}$ & FxCOR & n.s. & $38.569 * * *$ & 0.808 & \multirow[t]{4}{*}{ Partial } \\
\hline & $\mathrm{C}$ & FxINF & n.s. & $19.217^{*}$ & 0.555 & \\
\hline & $\mathrm{C}$ & FxINS & n.s. & n.s. & n.s. & \\
\hline & $\mathrm{C}$ & FxRM & n.s. & n.s. & n.s. & \\
\hline H8 & $\mathrm{C}$ & $\mathrm{L}$ & n.s. & $-21.517 * * *$ & 0.813 & Partial \\
\hline \multirow[t]{4}{*}{ H8a } & $\mathrm{C}$ & $\mathrm{LxCOR}$ & n.s. & $-23.563 * * *$ & 0.864 & \multirow[t]{4}{*}{ Partial } \\
\hline & $\mathrm{C}$ & LxINF & n.s. & n.s. & n.s. & \\
\hline & $\mathrm{C}$ & LxINS & n.s. & n.s. & n.s. & \\
\hline & $\mathrm{C}$ & LxRM & n.s. & n.s. & n.s. & \\
\hline
\end{tabular}

Table 20: Associations between Traits and Communicative Styles $\left({ }^{* * *} p<0.05,{ }^{* *} p<0.10,{ }^{*} p<0.15\right)$ 


\section{General Intelligence}

Again, the proportions of email messages classified according to each style were examined taking into account the apparent differences in estimates of G. These examinations lead to the following hypotheses. Again, it was proposed that communicative act moderated the association between style and the independent variable, G. Results of testing these hypotheses are presented in table 22 .

H9: $\mathrm{G}$ is negatively associated with proportion of team building style email messages sent, and:

H9a: This association is moderated by communicative act categories.

$\mathrm{H} 10$ : $\mathrm{G}$ is positively associated with proportion of hybrid style email messages sent, and:

H10a: This association is moderated by communicative act categories.

\begin{tabular}{|l|l|}
\hline T & Tentative \\
\hline TB & Team Building \\
\hline H & Hybrid \\
\hline F & Firm \\
\hline L & Lean \\
\hline C & Coordinating \\
\hline INF & Influencing \\
\hline INS & Instructing \\
\hline RM & Relationship Management \\
\hline
\end{tabular}

Table 21: Legend for Table 22 


\begin{tabular}{|c|c|c|c|c|c|c|}
\hline & Independent & Dependent & Rho & $\begin{array}{l}\text { Regression } \\
\text { Coefficient }\end{array}$ & $\mathbf{R}^{2}$ & Supported? \\
\hline H9 & $G$ & TB & n.s. & n.s. & n.s. & No \\
\hline \multirow[t]{4}{*}{ H9a } & $G$ & TBxCOR & n.s. & n.s. & $\overline{n . s .}$ & \multirow[t]{4}{*}{ Partial } \\
\hline & $\mathrm{G}$ & TBxINF & n.s. & n.s. & n.s. & \\
\hline & $\mathrm{G}$ & TBxINS & n.s. & $-0.461 *$ & 0.591 & \\
\hline & $\mathrm{G}$ & TBxRM & n.s. & $0.977 *$ & 0.570 & \\
\hline H10 & $\mathrm{G}$ & $\mathrm{H}$ & n.s. & n.s. & n.s. & No \\
\hline \multirow[t]{4}{*}{ H10a } & $\mathrm{G}$ & $\mathrm{HxCOR}$ & $0.90 * * *$ & n.s. & n.s. & \multirow[t]{4}{*}{ Yes } \\
\hline & $G$ & HxINF & n.s. & n.s. & n.s. & \\
\hline & $G$ & HxINS & n.s. & n.s. & n.s. & \\
\hline & $\mathrm{G}$ & HxRM & n.s. & n.s. & n.s. & \\
\hline
\end{tabular}

Table 22: Associations between $\mathbf{G}$ and Communicative Styles $\left({ }^{* * *} \mathbf{p}<0.05,{ }^{* *} \mathbf{p}<0.10,{ }^{*} \mathbf{p}<0.15\right)$.

Emotional Intelligence

MSCEIT branch scores and overall EI (EIQ) are shown for each team member in table 23. Reliabilities for individual tests are lower than the reliability of branch scores and so they are not shown here nor are they used in hypothesis testing. For more detailed score information, including empirical percentile scores and qualitative assessments, see appendix F.

\begin{tabular}{|l|l|c|c|c|c|c|}
\hline Ability & Coded As & Jason & Chris & Brian & Daniel & John \\
\hline $\begin{array}{l}\text { Perceiving } \\
\text { Emotions }\end{array}$ & PER & 79.245 & 85.282 & 86.768 & 93.886 & 95.521 \\
\hline $\begin{array}{l}\text { Facilitating } \\
\text { Thought }\end{array}$ & USE & 121.721 & 88.327 & 91.183 & 97.608 & 96.790 \\
\hline $\begin{array}{l}\text { Understanding } \\
\text { Emotions }\end{array}$ & UND & 95.115 & 85.306 & 107.127 & 108.287 & 109.658 \\
\hline $\begin{array}{l}\text { Managing } \\
\text { Emotions }\end{array}$ & MNG & 99.421 & 92.562 & 107.533 & 105.397 & 105.236 \\
\hline Overall EI & EIQ & 93.814 & 83.997 & 96.147 & 102.713 & 103.590 \\
\hline
\end{tabular}

Table 23: EI Test, Branch and Overall Scores (Normally distributed, Average = 100, $\mathrm{SD}=15$ ). 
Once again, EI branch scores were considered in examining the email message style proportions. Again, the moderating effect of communicative act category on the association between EI scores and communicative style was hypothesized. In some cases, there appeared to be an effect between EI scores and email message style for only certain communicative acts. In these cases the interaction effect was tested but not the main effect. Also, it was noted that the understanding emotions branch scores within this sample were highly correlated with overall EIQ $(\mathrm{Rho}=1.0)$. Therefore, no hypotheses were generated to assess the association between EIQ and email message style as the observed effects would likely be similar for any obtained by examining the understanding emotions branch. This analysis resulted in the hypotheses below. The results are shown in table 25 .

H11: The perceiving emotions branch of the MSCEIT is negatively associated with proportion of firm style email messages sent, and:

H11a: This association is moderated by communicative act categories. H12: The perceiving emotions branch of the MSCEIT is positively associated with proportion of hybrid style email messages sent, and:

H12a: This association is moderated by communicative act categories. H13: The perceiving emotions branch of the MSCEIT is negatively associated with proportions of team building style instructing acts.

H14: The using emotions branch of the MSCEIT is positively associated with proportions of team building style email messages sent, and:

H14a: This association is moderated by communicative act categories. 
H15: The understanding emotions branch of the MSCEIT is negatively associated with proportions of team building style instructing acts.

H16: The understanding emotions branch of the MSCEIT is positively associated with proportions of team building style relationship management acts.

H17: The understanding emotions branch of the MSCEIT is positively associated with proportions of firm style instructing acts.

H18: The managing emotions branch of the MSCEIT is positively associated with proportions of team building style relationship management acts.

H19: The managing emotions branch of the MSCEIT is negatively associated with proportions of tentative style relationship management acts.

H20: The managing emotions branch of the MSCEIT is negatively associated with proportions of tentative style instructing acts.

\begin{tabular}{|l|l|}
\hline T & Tentative \\
\hline TB & Team Building \\
\hline H & Hybrid \\
\hline F & Firm \\
\hline L & Lean \\
\hline C & Coordinating \\
\hline INF & Influencing \\
\hline NS & Instructing \\
\hline RM & Relationship Management \\
\hline
\end{tabular}

Table 24: Legend for Table 25 


\begin{tabular}{|c|c|c|c|c|c|c|}
\hline & Independent & Dependent & Rho & $\begin{array}{l}\text { Regression } \\
\text { Coefficient }\end{array}$ & $\mathbf{R}^{2}$ & Supported? \\
\hline H11 & PER & $\mathrm{F}$ & n.s. & n.s. & n.s. & No \\
\hline \multirow[t]{4}{*}{ H11a } & PER & FxCOR & n.s. & n.s. & n.s. & \multirow[t]{4}{*}{ Yes } \\
\hline & PER & FxINF & n.s. & n.s. & n.s. & \\
\hline & PER & FxINS & $0.80^{*}$ & n.s. & n.s. & \\
\hline & PER & FxRM & n.s. & n.s. & n.s. & \\
\hline H12 & PER & $\mathrm{H}$ & n.s. & $0.188^{*}$ & 0.950 & Partial \\
\hline \multirow[t]{4}{*}{ H12a } & PER & HxCOR & $1.0^{* * *}$ & $0.252 * *$ & 0.654 & \multirow[t]{4}{*}{ Yes } \\
\hline & PER & $\mathrm{HxINF}$ & n.s. & n.s. & n.s. & \\
\hline & PER & HxINS & $-0.821 * *$ & n.s. & n.s. & \\
\hline & PER & HxRM & n.s. & n.s. & n.s. & \\
\hline H13 & PER & TBxINS & $-0.90 * * *$ & n.s. & n.s. & Yes \\
\hline H14 & USE & TB & n.s. & n.s. & n.s. & No \\
\hline \multirow[t]{4}{*}{ H14a } & USE & TBXCOR & n.s. & n.s. & n.s. & \multirow[t]{4}{*}{ Partial } \\
\hline & USE & TBxINF & n.s. & $0.984 * * *$ & 0.888 & \\
\hline & USE & TBxINS & n.s. & n.s. & n.s. & \\
\hline & USE & TBxRM & n.s. & n.s. & n.s. & \\
\hline H15 & UND & TBxINS & $-1.0 * * *$ & $-0.624 * * *$ & 0.945 & Yes \\
\hline H16 & UND & TBxRM & $0.821 * *$ & $1.324 * * *$ & 0.862 & Yes \\
\hline H17 & UND & FxINS & $0.90 * * *$ & $2.229 * *$ & 0.709 & Yes \\
\hline H18 & $\mathrm{MNG}$ & TBxRM & $0.821 * *$ & $2.298 * * *$ & 0.863 & Yes \\
\hline H19 & MNG & TxRM & $-0.894 * * *$ & $-1.626 * * *$ & 0.808 & Yes \\
\hline H20 & $\mathrm{MNG}$ & TxINS & n.s. & $-2.169 * *$ & 0.657 & Partial \\
\hline
\end{tabular}

Table 25: Associations between EI Branch Scores and Communicative Styles $\left({ }^{* * *} \mathbf{p}<0.05,{ }^{* *} \mathbf{p}<0.10\right.$, ${ }^{*} \mathbf{p}<\mathbf{0 . 1 5}$ ).

\subsection{Summary}

Tables 26 and 28 summarize the significant findings of this study. Following this is a brief evaluation of the overall model which highlights the association between individual differences and email message style content, moderated by communicative act. A more detailed discussion of the overall findings and their implications for the study of EI in virtual teams is the subject of the subsequent section. 


\begin{tabular}{|l|c|c|l|}
\hline & Independent & Dependent & \multicolumn{1}{|c|}{ Rho } \\
\hline H1 & $\mathrm{N}$ & $\mathrm{T}$ & $-0.900^{* * *}$ \\
\hline \multirow{3}{*}{ H1a } & $\mathrm{N}$ & TxCOR & $-0.800^{*}$ \\
\cline { 2 - 4 } & $\mathrm{N}$ & TxINF & $-0.900^{* * *}$ \\
\cline { 2 - 4 } & $\mathrm{N}$ & TxINS & $-0.900^{* * *}$ \\
\hline H2 & $\mathrm{N}$ & $\mathrm{TB}$ & $1.000^{* * *}$ \\
\hline H3a & $\mathrm{N}$ & TBxCOR & $1.000^{* * *}$ \\
\cline { 2 - 4 } H5a & $\mathrm{N}$ & TBxINF & $0.900^{* * *}$ \\
\hline H6a & $\mathrm{E}$ & HxCOR & $-1.000^{* * *}$ \\
\hline H7 & $\mathrm{A}$ & FxINF & $-0.900^{* * *}$ \\
\hline H10a & $\mathrm{C}$ & LxINS & $0.890^{* * *}$ \\
\hline H11a & PER & F & $0.900^{* * *}$ \\
\hline H12a & PER & HxCOR & $0.900^{* * *}$ \\
\cline { 2 - 4 } & PER & HxCOR & $0.800^{*}$ \\
\hline H13 & PER & TBxINS & $-0.000^{* * *}$ \\
\hline H15 & UND & TBxINS & $-0.901^{* * *}$ \\
\hline H16 & UND & TBxRM & $0.821^{* *}$ \\
\hline H17 & UND & FxINS & $0.900^{* * *}$ \\
\hline H18 & MNG & TBxRM & $0.821 * *$ \\
\hline H19 & MNG & TxRM & $-0.894 * * *$ \\
\hline
\end{tabular}

Table 26: Summary of Significant Correlations $\left({ }^{* * *} \mathbf{p}<0.05,{ }^{* *} p<0.10,{ }^{*} \mathbf{p}<0.15\right)$.

\begin{tabular}{|l|l|}
\hline T & Tentative \\
\hline TB & Team Building \\
\hline H & Hybrid \\
\hline F & Firm \\
\hline L & Lean \\
\hline C & Coordinating \\
\hline INF & Influencing \\
\hline INS & Instructing \\
\hline RM & Relationship Management \\
\hline PER & Perceiving Emotions \\
\hline USE & Using Emotions \\
\hline UND & Understanding Emotions \\
\hline MNG & Managing Emotions \\
\hline
\end{tabular}

Table 27: Legend for Tables 26 and 28 


\begin{tabular}{|c|c|c|c|c|}
\hline & Independent & Dependent & $\begin{array}{l}\text { Regression } \\
\text { Coefficient } \\
\end{array}$ & $\mathbf{R}^{2}$ \\
\hline H1 & $\mathrm{N}$ & $\mathrm{T}$ & $-6.429 * * *$ & 0.909 \\
\hline \multirow[t]{3}{*}{ H1a } & $\mathrm{N}$ & TxCOR & $-4.232 * * *$ & 0.861 \\
\hline & $\mathrm{N}$ & TxINF & $-15.177 * * *$ & 0.742 \\
\hline & $\mathrm{N}$ & TxINS & $-12.722 * *$ & 0.684 \\
\hline $\mathrm{H2}$ & $\mathrm{N}$ & TB & $9.809 * * *$ & 0.909 \\
\hline \multirow[t]{2}{*}{ H2a } & $\mathrm{N}$ & TBxCOR & $14.028 * * *$ & 0.928 \\
\hline & $\mathrm{N}$ & TBxINF & $0.9848 * * *$ & 0.888 \\
\hline H3 & $E$ & $\mathrm{H}$ & $1.279 *$ & 0.647 \\
\hline \multirow[t]{2}{*}{ H3a } & $\mathrm{E}$ & HxCOR & $-2.547 * * *$ & 0.982 \\
\hline & $E$ & HxINS & $6.211 * *$ & 0.719 \\
\hline H5 & $\mathrm{A}$ & $\mathrm{F}$ & $-22.547 * *$ & 0.677 \\
\hline \multirow[t]{2}{*}{ H5a } & $\mathrm{A}$ & FxCOR & $-24.228^{*}$ & 0.556 \\
\hline & $\mathrm{A}$ & FxINF & $-18.274 * * *$ & 0.875 \\
\hline H6 & $\bar{A}$ & $\bar{L}$ & $14.870 * *$ & 0.677 \\
\hline H6a & $\mathrm{A}$ & $\mathrm{LxCOR}$ & $16.454 * *$ & 0.735 \\
\hline H7 & $\mathrm{C}$ & $\mathrm{F}$ & $34.006 * * *$ & 0.882 \\
\hline \multirow[t]{2}{*}{$\mathbf{H 7 a}$} & $\mathrm{C}$ & FxCOR & $38.569 * * *$ & 0.808 \\
\hline & $\mathrm{C}$ & FxINF & $19.217 *$ & 0.555 \\
\hline H8 & $\mathrm{C}$ & $\mathrm{L}$ & $-21.517 * * *$ & 0.813 \\
\hline H8a & $\mathrm{C}$ & LxCOR & $-23.563 * * *$ & 0.864 \\
\hline \multirow[t]{2}{*}{ H9a } & $\mathrm{G}$ & TBxINS & $-0.461 *$ & 0.591 \\
\hline & $\mathrm{G}$ & TBxRM & $0.977^{*}$ & $\overline{0.57}$ \\
\hline H12 & PER & $\mathrm{H}$ & $1.88 *$ & 0.95 \\
\hline H12a & PER & $\mathrm{HxCOR}$ & $2.52 * *$ & 0.654 \\
\hline H14a & USE & TBXINF & $9.84^{* * *}$ & 0.888 \\
\hline H15 & UND & TBxINS & $-6.24 * * *$ & 0.945 \\
\hline H16 & UND & TBxRM & $13.24 * * *$ & 0.862 \\
\hline H17 & UND & FxINS & $22.29 * *$ & 0.709 \\
\hline H18 & MNG & TBxRM & $22.98 * * *$ & 0.863 \\
\hline H19 & $\mathrm{MNG}$ & TxRM & $-16.26^{* * *}$ & 0.808 \\
\hline H20 & $\mathrm{MNG}$ & TxINS & $-21.69 * *$ & 0.657 \\
\hline
\end{tabular}

Table 28: Summary of Significant Regressions $\left({ }^{* * *} p<0.05, * * p<0.10,{ }^{*} p<0.15\right)$.

These results generally support the proposed model of the association between individual differences, consisting of Big 5 traits, G, EI and communicative style, moderated by communicative act. A few main effects were observed in the correlations. However, many more significant effects were observed when communicative styles were 
observed separately for each communicative act. It is furthermore proposed that a test with greater power may uncover other relationships which did not achieve significance in this study. These results and their implications are discussed in further detail in the next section. 


\section{CHAPTER 7 - DISCUSSION}

Each of the significant results of the study is discussed here taking into consideration theories and findings from $\mathrm{CMC}$, virtual team, EI and personality research. Also provided are some general observations from the data which suggest potentially valuable areas for future research. This discussion focuses on the manner in which ability based EI is evaluated using the MSCEIT and the extent to which it is relevant to research in virtual teams. A discussion of findings related to Big 5 personality traits is first presented followed by findings related to $\mathrm{G}$ and then EI. The discussion concludes with general observations which may provide important research avenues in the future.

\subsection{Findings Related to Personality Traits}

Neuroticism was found to have a strong negative association with the tentative style of email communication. This association held for coordinating, instructing and influencing acts, but was not significant for relationship management acts. Individuals possessing a greater degree of this trait are known to experience more frequent and higher levels of anxiety. Engaging in influencing or instructing acts could provide a source of anxiety given that changing the opinions of others and attempting to direct their activities could be a source of conflict. Given this, one might consider the tentative style to be used more frequently for these acts in order to reduce the perception that the message composer is applying force in altering the actions or beliefs of others. 
These findings, however, show the association to be in the opposite direction. One possible reason for this involves email rehearsability. While composing instructing or influencing acts, the sender may review the contents of their message and become concerned with that the action will not be performed or their opinions not duly considered. As a result, the individual with higher trait neuroticism may be inclined to increase the force of their statements by eliminating tentative style words. If this is true, email message content for these acts may differ significantly from the style employed in verbal communication. It is also possible that this effect is moderated by extraversion although the current study was unable to detect such an effect, if indeed it exists.

Assessing the negative association between neuroticism and tentative style coordinating acts requires an understanding of the fundamental difference between this act and those already discussed. In a coordinating act, the communicators typically commit themselves to performing an action dependent upon actions performed by others (Te'eni, 2001). The propensity for experiencing anxiety as a result of higher levels of neuroticism may reduce the motivation of a message sender engaging in a coordinating act to commit themselves to tasks where they require actions to be performed by others. As a result, they may choose to employ a more tentative style so as not to commit fully. However, the act still requires the management of interdependent actions to achieve goals and therefore the more anxious individual may prefer not to use the tentative style for fear that the end result may not be achieved.

Given these results, it could be theorized that more complex acts, such as influencing, may result in lower proportions of tentative style messages than less complex acts such as instructing. The regression coefficient for the relationship between 
neuroticism and tentative influencing acts has the greatest magnitude. However, the smallest regression coefficient amongst these is for coordinating which is likely a more complex act than instructing.

In these results, neuroticism is positively associated with team building style messages with significant relationships for coordinating and influencing acts. The regression coefficient found for the relationship between neuroticism and team building style influencing acts, however, represents only a one percent change in proportions of these types of messages for each unit increase in neuroticism scores which is not substantively significant. If the tentative style is not preferred by individuals higher in neuroticism, then perhaps the team building style is the best available alternative.

As suggested earlier, the team building style may be considered as email coming from a team identity rather than an individual. The SIDE model (Spears \& Lea, 1994) posits that $\mathrm{CMC}$ users form less distinct identities from those with whom they communicate. If this theory holds, one might expect that the team building style would be used by all members of a virtual team, regardless of personality traits. The current study, however, only found significant relationships with the trait neuroticism and some branches of EI which are discussed later. The SIDE effect may exist in virtual teams but it may also be moderated by individual differences such as traits.

A strong negative relationship was found between extraversion and hybrid coordinating acts. The main effect for the hybrid style was not significant. The magnitude of this effect does not appear large with a regression coefficient of approximately -2.5 . Hybrid messages consist of roughly equal proportions of the firm and lean style expressions. Also, coordinating actions typically involve committing oneself to 
performing an action under the assumption, or explicit request, that others will perform related actions. According to SIP (Walther, 1992; 1995), CMC communicators engage in more direct information seeking than would be performed in FtF interaction. One might expect that those with higher levels of extraversion would exhibit more communications consistent with this theory. Therefore extraverts may be more inclined to engage in firm style email communication although this effect was not significant in the current study.

Two strong associations were found between the trait agreeableness and communicative styles, although with no main effect. The relationship between this trait and firm style influencing acts was strongly negative with a large regression coefficient. This is what one would most likely expect given that agreeable individuals are motivated to consider the opinions of others (McCrae \& Costa, 1987) rather than firmly stating their own opinions which may differ from those of others. However, what these results cannot show is whether this effect is of the same, greater or lesser magnitude in CMC than in FtF interaction. It may be that medium does not moderate this effect but this requires further testing.

The relationship between trait agreeableness and lean style instructing messages was strongly positive but the regression coefficient was not significant. Lean messages are those that have the fewest, if any, equivalents in $\mathrm{FtF}$ interaction. There is no clear explanation for this relationship other than perhaps that an instructing action with very little content may be perceived by agreeable individuals as the least obtrusive way to request that someone perform an action.

The only significant association with the trait conscientiousness found was the main effect, although significant and large regression coefficients were found for 
coordinating and influencing acts employing this style. Overall, it is logical to predict that conscientious individuals would send the greatest proportion of firm style messages. Conscientious individuals tend to perform tasks thoroughly and persevere until their objectives are met. These individuals may also expect that others will do the same and therefore style their messages firmly when engaging in coordinating and influencing. However, this effect may hold only when they perceive others as conscientious. In the team studies here, no individual scored low in conscientiousness although the lowest scorer self-reported that he had less patience for highly detailed work than other members of the team.

It is not clear whether this effect holds for both virtual and non-virtual work arrangements. In the telephone interviews, all members of the team professed a belief that the work they were doing was important and perceived others to share that belief. It could be, therefore, that a team with a strong identity, which shares a common vision, engages in firm communication. This may, however, only be true if all members of the team exhibit high levels of conscientiousness.

\subsection{Findings Related to General Intelligence}

G correlated significantly with only hybrid style coordinating acts. The association was positive but the regression coefficient was not significant. G was included in this study to compare with observed effects of EI. G correlated strongly with emotional management branch scores in this study. However, there was no similar correlation between those branch scores and hybrid style coordinating acts. There was an 
observed positive correlation between the emotional perception branch scores and hybrid correlation but of similar magnitude. It is therefore difficult to say whether association with hybrid coordinating acts is greater for $\mathrm{G}$ or for emotional perception. A test with greater power may uncover smaller effects however it can also not be ruled out that $\mathrm{G}$ does not have many large influences on email message style proportions. Given that $\mathrm{G}$ has been shown to be distinct from EI (Mayer et al., 1999), and this study did not find evidence to counter this claim, no further discussion is warranted with respect to these results.

\section{$\underline{7.3 \text { Findings Related to Emotional Intelligence }}$}

The ability to perceive emotions, as assessed by the MSCEIT, correlated positively with firm style instructing actions and hybrid style coordinating. Its association with hybrid and team building style instructing actions was negative. In FtF interaction individuals performing an instructive act of communication may be inclined to alter the delivery of their message in accordance with perceptions of the recipient's emotional state. In CMC, the message sender's perceptions of others' emotions may not be as readily observed. Communications occurring before the instructing act may have shaped the message sender's perceptions of the emotional state of the recipient but it is unclear whether these perceptions remain constant over a given period of time. It could be that emotional perceptions are altered with each message sent in CMC as opposed to a more fluid change achieved through observation of emotional cues such as facial 
expression and vocal tones in FtF interaction. It is also unclear how accurately CMC communicators are able to detect the emotional state of one another.

If one perceived another as being in a bad mood they may change the wording of their instructing action slightly. 'Could you please do this' as opposed to 'Do this', for example. Therefore perceptions of another's emotional state may partially influence message style. Given the positive relation between ability to perceive emotions and firm style instructing acts, and the negative relation with other styles, it is possible that CMC users engage in different styles of instructing than FtF communicators. It is also possible, however, that the ability to perceive emotions, as measured by the MSCEIT, is not as useful in CMC as it is in FtF interaction. The low or non-significant correlations offer partial evidence that this may be true. The applicability of the perceiving emotions branch of EI is examined more fully in the final section of this discussion.

The emotional facilitation, or 'using', branch of EI was not significantly correlated with any message style or interaction between style and act. One significant positive regression coefficient was found between the ability to use emotions and team building style influencing acts. Of all the message styles, the team building style was observed to contain the most explicit emotional encoding. Also, those who are better able to use emotions are aware of which emotions are helpful in facilitating their thought processes for a given task. It may be that higher ability to use emotions motivates individuals to influence the emotional state of others in a manner consistent with their objectives. For example, such individuals may be motivated to use emotional expressions in highlighting the importance of taking a certain course of action for the overall benefit of the team. It is also possible that the individual is attempting to transfer their own 
emotional state to others so they will be more likely to be influenced by the opinions suggested.

The ability to understand emotions, their causes and the process through which they change was found to negatively correlate with team building style instructing acts. Instructing acts exhibit less affective complexity than other acts such as influencing (Te'eni, 2001). Therefore, it may be that those who better understand emotions deem an affective strategy, such as team building, to be inappropriate. This effect however is somewhat moderate according to the observed regression coefficient $(-0.624)$.

A much larger effect was found for the positive relationship between understanding emotions and team building style relationship management acts. Of all the communicative acts, relationship management accounts for the greatest proportion of team building style messages sent. This suggests that in managing affective relationships, the team building style is deemed most appropriate by those with higher ability to understand emotions. Another way to view this relationship is that those who have higher abilities in this branch of EI may be more effective in establishing relationships with others. Such individuals may be better suited to leadership of virtual teams and this view is consistent with at least one leadership study (Rosete \& Ciarrochi, 2005).

Another positive association was found between the ability to understand emotions and firm style instructing actions. Here again the effect size is large with a regression coefficient of 2.229. This effect is similar to that observed for the emotional perception branch suggesting that those higher in EI prefer the firm style when engaging in the relatively uncomplicated act of instructing. It should also be noted however that 
both these branches were highly correlated within this sample and therefore some similar associations can be expected.

The ability to manage emotions in the self and others was found to associate positively with the team building style of relationship management act and the regression coefficient was large (2.298). Given that this effect is similar to that observed for the understanding emotions branch, these two abilities may be complementary in motivating an individual to engage in this style of communication when performing acts of relationship management. There is little reason to assume that this effect would be substantially different in FtF interactions. Therefore, it may be that individuals are able to engage in this style of communication regardless of whether the medium of communication is $\mathrm{FtF}$ or $\mathrm{CMC}$. Further testing is warranted to test this possibility given the perception of lack of immediacy in CMC.

Finally, a strong negative association was found between the ability to manage emotions and the tentative style of relationship management acts. Consistent with the finding that the team building style is preferred for those with higher emotional management ability, the tentative style may be the least preferred method of performing this communicative act. Those who are better able to manage emotions, and to understand them, should be more confident in managing relations with others and this effect may also be influenced by extraversion, although this was not found in the current study. 


\subsection{General Findings Concerning EI}

A further observation was made in this study which was not initially sought. According to George (1990), who provided the first account of the concept of group affect, teams tend to select members with characteristics similar to their own. While that study focused on personality traits of individuals, it is also conceivable that teams might select or attract members with similar EI abilities given the role of EI in social responding (Lopes, et al., 2005). In other words, individuals may feel more comfortable working amongst others whose emotional abilities are similar to their own. The variance in branch scores amongst the members of this team was within roughly one standard deviation (15 points) with the exception of managing emotions which exhibited about two standard deviations in range. Scores for neuroticism, extraversion and openness to experience exhibited fairly high variance while all members were moderately to highly agreeable and conscientious. The variance across traits within the team is very close to what Neuman, Wagner and Christianson (1999) predicted would lead to effective performance. These results may lead to the tentative proposition that EI abilities may be significant predictors of the group affect construct in addition to the personality traits of those working in a virtual team.

A final observation concerns the measurement of emotional perception and its applicability to virtual team research. In the MSCEIT, this ability is assessed through two tasks, one involving the recognition of emotion in facial expressions and the other through observation of images. It is not possible to ascertain whether the ability assessed through this technique is applicable to those who interact primarily through CMC. The 
ability to perceive emotions from email messages may not be fully captured by the MSCEIT. In order to fully assess this ability in a virtual team where FtF interaction is rare, a different type of test may be needed. For example, the ability to perceive emotions where the communicative style of a message sender differs from their usual style may be developed by those who have greater experience in using this medium. Also, it was noted that ability to perceive emotions was somewhat low for every team member. Further studies are required to ascertain whether one's ability to perceive emotions, especially in facial expressions, is potentially reduced when these individuals work for extended periods in a virtual team. 


\section{CHAPTER 8 - SUMMARY AND CONCLUSIONS}

Research into EI has uncovered several desirable qualities and outcomes for which it accounts. Individuals who are more aware of emotions in themselves and others and are also able to understand and manage emotions may form more positive and effective relationships with others. These individuals may also be better at inducing positive emotions in others which is useful in creating and maintaining a team which handles conflict more effectively and with less damage to interpersonal relationships. Perhaps these individuals are better able to establish, maintain or repair trust and be more effective in serving as emergent leaders through displaying context appropriate emotional expressions.

The list of potential effects could be quite extensive. Indeed, what limit is there to the results of applying human cognitive abilities to a given task? But EI effects are largely unknown in virtual teams. Such teams are faced with potentially less communication, especially of an informal nature, for which more opportunities may arise when individuals encounter each other in regular FtF interaction.

How do individuals who are better able to detect emotions in others detect them when the only available communication medium is email? Similar questions can be raised regarding the ability to use and manage emotions. The answers to these questions are part of a research agenda for which this exploratory study offers systematic guidance. If EI abilities can be used in interactions with virtual team members, then the evidence must largely be contained within the content of their email communications. 
This study was motivated to find this evidence and use it to construct a framework for research, grounded in both data and theory. It was found that EI abilities, as well as individual differences in personality traits and, to a lesser extent $G$, influenced the chosen style of email communication dependent upon the intent of the communicator. Having completed the exploratory objectives of this study, it is apparent that further research may prove valuable in understanding the role of EI in virtual teams and how the abilities derived from it may be used similarly or differently than in FtF interaction.

The exploratory nature of this study may limit the generalizability of any findings and, therefore, a full assessment of its validity is not possible. A substantial amount of data was collected, but from only a single virtual team. This team has existed for many years and therefore evidence of the process through which the members have established norms of communication may not be observed in the data set. As each virtual team exists within its own context, and is comprised of members possessing various preferences, abilities and personalities, the norm formation process will likely have many different outcomes. Future studies are necessary in which several teams are included so that between person, and between group differences, and the sources for those differences, may be analyzed.

As the team consists of only five members, the significant results of hypothesis testing include only those with the largest effect size. Nonetheless, the positive findings of this study suggest that further research is warranted into the influence of EI and other individual differences on email message content. A greater sample size in future studies will aid in verifying the results of this study as well as uncovering effects not detected in the current sample. 
Due to power limitations, it was not possible to fully assess the extent to which personality traits and $\mathrm{G}$ interact with $\mathrm{EI}$ to influence the content of email messages. Given that these results do not appear to indicate that individual differences are equivalently associated with the styles of email messages produced by a virtual team, further research is again warranted. A test with greater power will be able to assess differing strengths of association as well as potential mediating or moderating effects.

These limitations aside, this study has much to offer both in terms of both theory and practice. Both research into virtual teams and EI are well established avenues within academic literature but have never been examined in the fashion outlined in this study. This study provides valuable evidence concerning the extent to which EI abilities, as well as individual differences in personality traits and general intelligence, influence the contents of email messages. In addition, evidence is provided to suggest that the manner in which certain abilities are used may differ in $\mathrm{CMC}$ interaction as compared to $\mathrm{FtF}$ interaction. This evidence supports the contention that further research is warranted in the area of EI and virtual teams. 


\section{REFERENCES}

Ahuja, M. \& Carley, K. (1999). Network structure in virtual organizations. Organization Science, 10, 741-757.

Armon-Jones, C. (1986). The thesis of constructionism. In R. Harre, R. (Ed.), The social construction of emotions (pp. 32-56). Oxford: Blackwell.

Ashforth, B.E. \& Humphrey, R.H. (1993). Emotional labor in service roles: The influence of identity. Academy of Management Review, 18, 88-115.

Ashforth, B.E. \& Kreiner, G.E. (2002). Normalizing emotion in organizations: Making the extraordinary seem ordinary. Human Resource Management Review, 12, 215-235.

Ashkansy, N.M. \& Dasborough, M.T. (2003). Emotional awareness and emotional intelligence in leadership teaching. Journal of Education for Business, 79, 18-22.

Ashkanasy, N.M. \& Daus, C.S. (2005). Rumors of the death of emotional intelligence in organizational behavior are vastly exaggerated. Journal of Organizational Behavior, $26,441-452$.

Ashkanasy, N.M., Hartel, C.E.J. \& Daus, C.S. (2002). Diversity and emotion: The new frontiers in organizational behavior research. Journal of Management, 28, 307-338.

Ayoko, O.B., Callan, V.J. \& Hartel, C.E.J. (2003). Workplace conflict, bullying, and counterproductive behaviours. The International Journal of Organizational Analysis, $11,283-301$.

Barchard, K.A. (2003). Does emotional intelligence assist in the prediction of academic success? Educational and Psychological Measurement, 63, 840-858.

Barchard, K.A. \& Hakstian, A.R. (2004). The nature and measurement of emotional intelligence abilities: Basic dimensions and their relationships with other cognitive ability and personality variables. Educational and Psychological Measurement, 64, $437-462$.

Bar-On, R. Emotional and social intelligence. (2000). Emotional and social intelligence: Insights from the emotional quotient inventory. In R. Bar-On \& J.D.A. Parker (Eds.) The handbook of emotional intelligence (pp. 363-388). San Francisco: Jossey-Bass.

Bar-On, R. (1997). Bar-On emotional quotient inventory (EQ-i): Technical Manual. Toronto, Canada: Multi-Health Systems. 
Barret, M., Jarvenpaa, S., Silva, L. \& Walsham, G. (2003). ICTs, globalization and local diversity. Communications of the Association for Information Systems, 11, 486-497.

Bartel, C.A. \& Saavedra, R. (2000). The collective construction of work group moods. Administrative Science Quarterly, 45, 197-231.

Bass, B.M. (1985). Leadership: Good, better, best. Organizational Dynamics, 13, 26-40.

Beersma, B., Harinck, F. \& Gerts, M.J.J. (2003). Bound in honor: How honor values and insults affect the experience and management of conflicts. The International Journal of Conflict Management, 14, 75-94.

Boland Jr., R.J. (1996). Why shared meanings have no place in structuration theory: A reply to Scapens and MacIntosh. Accounting, Organizations and Society, 21, 691697.

Boland Jr., R.J. (1991). Information system use as a hermeneutic process. In H.-E. Nissen, H.K. Klein \& R. Hirschheim (Eds.) Information systems research: Contemporary approaches \& emergent traditions (pp. 439-458). Amsterdam: Elsevier Science Publishers B.V.

Boland Jr., R.J. \& Day, W.F. (1989). The experience of system design: A hermeneutic of organizational action. Scandinavian Journal of Management, 5, 87-104.

Boonthanom, R. (2004). Computer-mediated communication of emotions: A lens model approach. (Unpublished Ph.D. Dissertation), Florida State University.

Boyatzis, R.E., Goleman, D., \& Rhee, K.S. (2000). Clustering competence in emotional intelligence. Insights from the emotional competence inventory. In R. Bar-On \& J.D.A. Parker (Eds.) The handbook of emotional intelligence. San Francisco: JosseyBass.

Brackett, M.A. \& Mayer, J.D. (2003). Convergent, discriminant, and incremental validity of competing measures of emotional intelligence. Personality and Social Psychology Bulletin, 29, 1147-1158.

Brackett, M.A., Mayer, J.D. \& Warner, R.M. (2004). Emotional intelligence and its relation to everyday behaviour. Personality and Individual Differences, 36, 1387 1402 .

Brannan, M.J. (2005). Once more with feeling: Ethnographic reflections on the mediation of tension in a small team of call centre workers. Gender, Work and Organization, 12, 420-439. 
Brief, A.P., Butcher, A.H. \& Roberson, L. (1995). Cookies, disposition, and job attitudes: The effects of positive mood-inducing events and negative affectivity on job satisfaction in a field experiment. Organizational Behavior and Human Decision Processes, 62, 55-62.

Brief, A.P. \& Weiss, H.M. (2002). Organizational behavior: Affect in the workplace. Annual Review of Psychology, 53, 279-307.

Brown, H.G., Poole, M.S. \& Rodgers, T.L. (2004). Interpersonal traits, complementarity, and trust in virtual collaboration Journal of Management Information Systems, 20, 115-137.

Brown, S.P., Westbrook, R.A. \& Challagalla, G. (2005). Good cope, bad cope: Adaptive and maladaptive coping strategies following a critical negative work event. Journal of Applied Psychology, 90, 792-798.

Burgoon, J.K., Bonito, A.B., Ramirez Jr., A., Dunbar, N.E., Kam, K. \& Fischer, J. (2002). Testing the interactivity principle: Effects of mediation, propinquity, and verbal and nonverbal modalities in interpersonal interaction. Journal of Communication, 52, 657-677.

Burnett, G. (2002). The scattered members of an invisible republic: Virtual communities and Paul Ricoeur's hermeneutics. The Library Quarterly, 72, 155-178.

Burnett, G., Dickey, M.H., Kazmer, M.M. \& Chudoba, K.M. (2003). Understanding virtual community through language: A cultural hermeneutic examination. Academy of Management Best Conference Paper OCIS.

Butler, B. (2001). Memberships size, communication activity, and sustainability: A resource-based model of online social structures. Information Systems Research, 12, 346-362.

Carlson, J.R., George, J.F., Burgoon, J.K., Adkins, M. \& White, C.H. (2004). Deception in computer-mediated communication. Group Decision and Negotiation, 13, 5-28.

Carlson, J.R. \& Zmud, R.W. (1999). Channel expansion theory and the experiential nature of media richness perceptions. Academy of Management Journal, 42, 153-170.

Caruso, D.R. \& Wolfe, C.J. (2001). Emotional intelligence in the workplace. In J. Ciarrochi, J.P. Forgas \& J.D. Mayer (Eds.) Emotional intelligence in everyday life, Philadelphia, PA: Psychology Press.

Charmaz, K. (2000). Grounded theory in the $21^{\text {st }}$ century. In N.K. Denzin \& Y.S. Lincoln (Eds.) Handbook of qualitative research (pp. 507-535). Thousand Oaks, CA: Sage Publications. 
Cohen, J. (1960). A coefficient of agreement for nominal scales. Educational and Psychological Measurement, 20, 37-46.

Conte, J.M. (2005). A review and critique of emotional intelligence measures. Journal of Organizational Behaviour, 26, 433-440.

Coppola, N.W., Hiltz, S.R. \& Rotter, N.G. (2004). Building trust in virtual teams. IEE Transactions on Professional Communication, 47, 95-104.

Cote, S. \& Morgan, L.M. (2002). A longitudinal analysis of the association between emotion regulation, job satisfaction, and intentions to quit. Journal of Organizational Behavior, 23, 947-962.

Cramton, C.D. (2001). The mutual knowledge problem and its consequences for dispersed collaboration. Organization Science, 12, 346-371.

Daft, R.L. \& Lengel, R.H. (1986). Organizational information requirements, media richness and structural design. Management Science, 32, 554-571.

Daft, R., Lengel, R. \& Trevino, L. (1987). Message equivocality, media selection, and manager performance: Implications for information systems, MIS Quarterly, 11, 355366.

Dasborough, M.T. \& Ashkanasy, N.M. (2002). Emotion and attribution of intentionality in leader-member relationships. The Leadership Quarterly, 13, 615-634.

Daus, C.S. \& Ashkanasy, N.M. (2005). The case for the ability-based model of emotional intelligence in organizational behavior. Journal of Organizational Behavior, 26, 453466.

Day, A.L. \& Carroll, S.A. (2004). Using an ability-based measure of emotional intelligence to predict individual performance, group performance, and group citizenship behaviours. Personality and Individual Differences, 36, 1443-1458.

De Cindio, F., Gentile, O., Grew, P. \& Redolfi, D. (2003). Community Networks: Rules of behavior and social structure, The Information Society, 19, 395-406.

De Cremer, D., Stinglhamber, F. \& Eisenberger, R. (2005). Effects of own versus other's fair treatment on positive emotions: A field study. The Journal of Social Psychology, $145,741-744$.

Dennis, A.R. \& Kinney, S.T. (1998). Testing media richness theory in the new media: The effects of cues, feedback, and task equivocality. Information Systems Research, 9, 256-274. 
Dennis, A.R. \& Valacich, J.S. (1999). Rethinking media richness: Towards a theory of media synchronicity. Proceedings of the $32^{\text {nd }}$ Hawaii International Conference on System Sciences.

Denzin, N.K. (1999). Interpretive ethnography for the next century. Journal of Contemporary Ethnography, 28, 510-519.

Denzin, N.K. \& Lincoln, Y.S. (2000). The discipline and practice of qualitative research. In N.K. Denzin \& Y.S. Lincoln (Eds.) Handbook of qualitative research (pp. 1-32). Thousand Oaks, CA: Sage Publications.

De Raad, B. (2005). The trait-coverage of emotional intelligence. Personality and Individual Differences, 38, 673-687.

DeRosa, D.M., Hantula, D.A., Kock, N. \& D'Arcy, J. (2004). Trust and leadership in virtual teamwork: A media naturalness perspective. Human Resource Management, $43,219-232$.

DeSanctis, G. \& Monge, P. (1999). Introduction to the special issue: Communication processes for virtual organizations. Organization Science, 10, 693-703.

DeSanctis, G. \& Poole, M.S. (1994). Capturing the complexity in advanced technology use: Adaptive structuration theory. Organization Science, 5, 121-147.

Diefendorff, J,.M. \& Gosserand, R.H. (2003). Understanding the emotional labor process: A control theory perspective. Journal of Organizational Behavior, 24, 945-959.

Drodge, E.N. \& Murphy, S.A. (2002). Interrogating emotions in police leadership. Human Resource Development Review, 1, 420-438.

Duffy, M.K., Ganster, D.C. \& Shaw, J.D. (1998). Positive affectivity and negative outcomes: The role of tenure and job satisfaction. Journal of Applied Psychology, 83, 950-959.

Elfenbein, H.A. \& Ambady, N. (2002). Predicting workplace outcomes from the ability to eavesdrop on feelings. Journal of Applied Psychology, 87, 963-971.

Engelberg, E. \& Sjoberg, ,L. (2004). Internet use, social skills, and adjustment. CyberPsychology and Behavior, 7, 41-47.

Farkas, A.J. \& Tetrick, L.E. (1989). A three-wave longitudinal analysis of the causal ordering of satisfaction and commitment on turnover decisions. Journal of Applied Psychology, 74, 855-868.

Fineman, S. \& Sturdy, A. (1999). The emotions of control: A qualitative exploration of environmental regulation. Human Relations, 52, 631-663. 
Fisher, C.D. (2000). Mood and emotions while working: Missing pieces of job satisfaction? Journal of Organizational Behavior, 21, 185-202.

Fitness, J. (2000). Anger in the workplace: An emotion script approach to anger episodes between workers and their superiors, co-workers and subordinates. Journal of Organizational Behavior, 21, 147-162.

Friedman, R.A. \& Currall, S.C. (2003). Conflict escalation: Dispute exacerbating elements of e-mail communication. Human Relations, 56, 1325-1347.

Frost, P.J. (2004). Handling toxic emotions: New challenges for leaders and their organization. Organizational Dynamics, 33, 111-127.

Furnham, A. (2005). Gender and personality differences in self- and other ratings of business intelligence. British Journal of Management, 16, 91-103.

Gans, H.J. (1999). Participant observation in the era of 'ethnography'. Journal of Contemporary Ethnography, 28, 540-548.

Gefen, D. \& Straub, D.W. (1997). Gender differences in the perception and use of e-mail: An extension to the technology acceptance model. Management Information Systems Quarterly, 21, 389-400.

George, J.M. (1990). Personality, Affect, and Behavior in Groups. Journal of Applied Psychology, 75, 107-116.

George, J.M. \& James, L.R. (1993). Personality, affect, and behaviour in groups revisited: comment on aggregation, levels of analysis, and a recent application of within and between analysis. Journal of Applied Psychology, 78, 798-804.

Ghom, C.L., Corser, G.C. \& Dalsky, D.J. (2005). Emotional intelligence under stress: Useful, unnecessary, or irrelevant? Personality and Individual Differences, 39, 10171028.

Ghosh, T., Yates, J. and Orlikowski, W.J. (2004). Using communication norms for coordination: Evidence from a distributed team. Twenty-Fifth International Conference on Information Systems.

Gibson, C.B. \& Cohen, S.G. (2003). Virtual teams that work, San Francisco, CA: JosseyBass.

Glaser, B.G. \& Strauss, A.L. (1967). The discovery of grounded theory: Strategies for qualitative research, Hawthorne, NY: Aldine de Gruyter.

Glomb, T.M., Kammeyer-Mueller, J.D. \& Rotundo, M. (2004). Emotional labor demands and compensating wage differentials. Journal of Applied Psychology, 89, 700-714. 
Goleman, D. (1995) Emotional Intelligence. New York: Bantam.

Gopal, A., Bostrom, R.P. \& Chin, W.W. (1992-3). Applying adaptive Structuration theory to investigate the process of group support systems use. Journal of Management Information Systems, 9, 45-69.

Goulding, C. (2005). Grounded theory, ethnography and phenomenology. European Journal of Marketing, 39, 294-308.

Grabowski, M. \& Roberts, K. (1999). Risk mitigation in virtual organizations. Organization Science, 10, 704-721.

Guerrier, Y \& Adib, A. (2003). Work at leisure and leisure at work: A study of the emotional labour of tour reps. Human Relations, 56, 1399-1417.

Harris, L.C. (2002). The emotional labour of barristers: An exploration of emotional labour by status professionals. Journal of Management Studies, 39, 553-584.

Hertel, G., Geister, S. \& Konradt, U. (2005). Managing virtual teams: A review of current empirical research, Human Resource Management Review, 15, 69-95.

Higgs, M. \& Aitken, P. (2003). An exploration of the relationship between emotional intelligence and leadership potential. Journal of Managerial Psychology, 18, 814823.

Hinds, P.J. \& Bailey, D.E. (2003). Out of sight, out of sync: Understanding conflict in distributed teams. Organization Science, 14, 615-632.

Hinds, P.J. \& Mortensen, M. (2002). Understanding antecedents to conflict in geographically distributed research and development teams. Twenty-Third International Conference on Information Systems, 415-424.

Hochschild, A.R. (1983). The managed heart, Berkely and Los Angeles, CA: University of California Press.

Hoyt, C.L. \& Blascovich, J. (2003). Transformational and transactional leadership in virtual and physical environments. Small Group Research, 34, 678-715.

Ilies, R. \& Judge, T.A. (2004). An experience-sampling measure of job satisfaction and its relationships with affectivity, mood at work, job beliefs, and general job satisfaction. European Journal of Work and Organizational Psychology, 13, 367-389.

James, K.T. \& Arroba, T. (2005). Reading and Carrying: A framework for learning about emotion and emotionality in organizational systems as a core aspect of leadership development. Management Learning, 36, 299-316. 
Jarvenpaa, S.L., Knoll, K. \& Leidner, D.E. (1998). Is anybody out there? Antecedents of trust in global virtual teams. Journal of Management Information Systems, 14, 29-64.

Jarvenpaa, S.L. \& Leidner, D.E. (1999). Communication and trust in global virtual teams. Organization Science, 10, 791-815.

John, O.P. \& Srivistava, S. (1999). The big five inventory. In: L.A. Pervin \& O.P. John (Eds.) Handbook of personality: Theory and research (pp. 102-138), New York, NY: Guilford Press.

Jones, T.S. \& Bodtker, A. (2001). Mediating with heart and mind: Addressing emotion in mediation practice. Negotiation Journal, 17, 217-244.

Jordan, P.J. \& Troth, A.C. (2002). Emotional intelligence and conflict resolution: Implications for human resource development. Advances in Developing Human Resources, 4, 62-79.

Kafetsios, K. (2004). Attachment and emotional intelligence abilities across the life course. Personality and Individual Differences, 37, 129-145.

Kayworth, T.R. \& Leidner, D.E. (2001-2). Leadership effectiveness in global virtual teams. Journal of Management Information Systems, 18, 7-40.

Kelly. J.R. \& Barsade, S.G. (2001). Mood and emotions in small groups and work teams. Organizational Behavior and Human Decision Processes, 86, 99-130.

Kirkman, B.L., Rosen, B., Gibson, C.B., Tesluk, P.E. \& McPherson, S.O. (2002). Five challenges to virtual team success: Lessons from Sabre, Inc. Academy of Management Executive, 16, 67-79.

Korczynski, M. (2003). Communities of coping: Collective emotional labour in service work. Organization, 10, 55-79.

Kraut, R., Mukhopadhyay, T., Szczypula, J., Kiesler, S. \& Scherlis, B. (1999). Information and communication: Alternative uses of the internet in households. Information Systems Research, 10, 287-303.

Krippendorff, K. (2004). Content analysis: An introduction to its methodology, Thousand Oaks, CA: Sage Publications.

Kruml, S.M. \& Geddes, D. (2000). Exploring the dimensions of emotional labor. Management Communication Quarterly, 14, 8-49.

Lam, L.T. \& Kirby, S.L. (2002). Is emotional intelligence an advantage? An exploration of the impact of emotional and general intelligence on individual performance. The Journal of Social Psychology, 142, 133-143. 
Landy, F.J. (2005). Some historical and scientific issues related to research on emotional intelligence. Journal of Organizational Behaviour, 26, 411-424.

Law, K.S., Wong, C. \& Song, L.J. (2004). The construct and criterion validity of emotional intelligence and its potential utility for management studies. Journal of Applied Psychology, 89, 483-496.

LeBlanc, M.M. \& Kelloway, E.K. (2002). Predictors and outcomes of workplace violence and aggression. Journal of Applied Psychology, 87, 444-453.

Lee, A.S. (1994). Electronic mail as a medium for rich communication: An empirical investigation using hermeneutic interpretation. Management of Information Systems Quarterly, 18, 143-157.

Lee, A.S. (1991). Integrating positivist and interpretive approaches to organizational research. Organization Science, 2, 342-365.

Lee, K. \& Allen, N.J. (2002). Organizational citizenship behavior and workplace deviance: The role of affect and cognitions. Journal of Applied Psychology, 87, 131142.

Leenders, R.Th.A.J., vanEngelen, J.M.L. \& Kratzer, J. (2003). Virtuality, communication, and new product team creativity: A social network perspective. Journal of Engineering and Technology Management, 20, 69-92.

Lipnack, J. \& Stamps, J. (1997). Virtual teams: Reaching across space, time, and organizations with technology. New York: John Wiley \& Sons.

Livingstone, H.A. \& Day, A.L. (2005). Comparing the construct and criterion-related validity of ability-based and mixed-model measures of emotional intelligence. Educational and Psychological Measurement, 65, 757-779.

Locke, E.A. (2005). Why emotional intelligence is an invalid concept. Journal of Organizational Behaviour, 26, 425-431.

Lopes, P.N., Brackett, M.A., Nezlek, J.B., Schutz, A., Sellin, I. \& Salovey, P. (2004). Emotional intelligence and social interaction. Personality and Social Psychology Bulletin, 30, 1018-1034.

Lopes, P.N., Salovey, P., Cote, S. \& Beers, M. (2005). Emotion regulation abilities and the quality of social interaction. Emotion, 5, 113-118.

Lopes, P.N., Salovey, P. \& Straus, R. (2003). Emotional intelligence, personality, and the perceived quality of social relationships. Personality and Individual Differences, 35 , 641-658. 
Lyons, J.B. \& Schneider, T.R. (2005). The influence of emotional intelligence on performance. Personality and Individual Differences, 39, 693-703.

Majchrzak, A., Rice, R.E., Malhotra, A., King, N. \& Ba, S. (2000). Technology adaptation: The case of a computer-supported inter-organizational virtual team. MIS Quarterly, 24, 569-600.

Mandell, B. \& Pherwani, S. (2003). Relationship between emotional intelligenc and transformational leadership style: A gender comparison. Journal of Business and Psychology, 17, 387-404.

Mann, S. (1999). Emotion at work: To what extent are we expressing, suppressing, or faking it? European Journal of Work and Organizational Psychology, 8, 347-369.

Mann, S., Varey, R. \& Button, W. (2000). An exploration of the emotional impact of tele-working via computer-mediated communication. Journal of Managerial Psychology, 15, 668-690.

Markus, M.L. (1994). Electronic mail as the medium of managerial choice. Organization Science, 5, 502-525.

Markus, M.L. (1987) Toward a 'critical mass' theory of interactive media. Communication Research, 1987, 14, 491-511.

Martins, L.L., Gilson, L.L. \& Maynard, M.T. (2004). Virtual teams: What do we know and where do we go from here. Journal of Management, 30, 805-835.

Mathews, G., Roberts, R.D. \& Zeidner, M. (2004). Seven myths about emotional intelligence. Psychological Inquiry, 15, 179-196.

Mayer, J.D., Caruso, D.R., \& Salovey, P. (1999) Emotional intelligence meets traditional standards for an intelligence. Intelligence, 27, 267-298.

Mayer, J.D. \& Salovey, P. (1997). What is emotional intelligence? In P. Salovey \& D. Sluyter (Eds.), Emotional development and emotional intelligence: Educational implications (3-31). New York, NY: Basic Books.

Mayer, J.D., Salovey, P., \& Caruso, D.R. (1999) MSCEIT Item Booklet Toronto, Canada: Multi-Health Systems.

Mayer, J.D., Salovey, P., \& Caruso, D.R. (2002) MSCEIT User's Manual Toronto, Canada: Multi-Health Systems.

Mayer, J.D., Salovey, P. \& Caruso, D.R. (2004). Emotional Intelligence: Theory, Findings, and Implications. Psychological Inquiry, 15, 197-215. 
Mayer, J.D., Salovey, P., Caruso, D.R., \& Sitarenios, G. (2003). Measuring emotional intelligence with the MSCEIT V2.0. Emotion, 3, 97-105.

McColl-Kennedy, J.R. \& Anderson, R.D. (2002). Impact of leadership style and emotions on subordinate performance. The Leadership Quarterly, 13, 545-559.

McCrae, R.R \& Costa, Jr., P.T. (1987) Validation of the five-factor model of personality across instruments and observers. Journal of Personality and Social Psychology, 52, $81-90$

Mennecke, B.E., Valacich, J.S. \& Wheeler, B.C. (2000). The effects of media and task on user performance: A test of the task-media fit hypothesis. Group Decision and Negotiation, 9, 507-529.

Michie, S. \& Gooty, J. (2005). Values, emotions, and authenticity: Will the real leader please stand up? The Leadership Quarterly, 16, 441-457.

Mignonac, K. \& Herrbach, O. (2004). Linking work events, affective states, and attitudes: An empirical study of managers' emotions. Journal of Business and Psychology, 19, 221-240.

Miller, K. (2002). The experience of emotion in the workplace. Management Communication Quarterly, 15, 571-600.

Montoya-Weiss, M.M. , Massey, A.P. \& Song, M. (2001). Getting it together: Temporal coordination and conflict management in global virtual teams. Academy of Management Journal, 44, 1251-1262.

Morris. J.A. \& Feldman, D.C. (1996). The dimensions, antecedents, and consequences of emotional labor. Academy of Management Review, 21, 986-1010.

Mortensen, M. \& Hinds, P.J. (2001). Conflict and shared identity in geographically distributed teams. The International Journal of Conflict Management, 12, 212-238.

Mulholland, K. (2002). Gender, emotional labour and teamworking in a call centre. Personnel Review, 31, 283-303.

Mullen, B. \& Copper, C. (1994). The relation between group cohesiveness and performance: An integration. Psychological Bulletin, 115, 210-227.

Neuendorf, K.A. (2002). The contents analysis guidebook, Thousand Oaks, CA: Sage Publications.

Neuman, G.A., Wagner, S.H. \& Christianson, N.D. (1999). The relationship between work-team personality composition and the job performance of teams. Group and Organization Management, 24, 28-45. 
Ngwenyama, O.K. \& Lee, A.S. (1997) Communication richness in electronic mail: Critical social theory and the contextuality of meaning, MIS Quarterly, 21, 145-167.

Ogbonna, E. \& Harris, L.C. (2004). Work intensification and emotional labour among UK university lecturers an exploratory study. Organization Studies, 25, 1185-1203.

Organ, D.W. \& Near, J.P. (1985). Cognition vs affect in measures of job satisfaction. International Journal of Psychology, 20, 241-253.

Orlikowski, W.J. (1993). CASE tools as organizational change: Investigating incremental and radical changes in systems development. Management Information Systems Quarterly, 17, 310-340.

Orlikowski, W.J. \& Baroudi, J.J. (1991). Studying information technology in organizations: Research approaches and assumptions. Information Systems Research, 2, 1-28.

Panteli, N. (2004). Discursive articulations of presence in virtual organizing. Information and Organization, 14, 59-81.

Panteli, N. \& Sockalingam, S. (2005). Trust and conflict within virtual interorganizational alliances: A framework for facilitating knowledge sharing. Decision Support Systems, 39, 599-617.

Paul, D.L. \& McDaniel Jr., R.R. (2004). A field study of the effect of interpersonal trust on virtual collaborative relationship performance. MIS Quarterly, 28, 183-227.

Paul, S., Samarah, I.M., Seetharaman, P. \& Mykytyn Jr., P.P. (2004-5). An empirical investigation of collaborative conflict management style in group support systembased global virtual teams. Journal of Management Information Systems, 21, 185222.

Pauleen, D.J. (2003-4). An inductively derived model of leader-initiated relationship building with virtual team members. Journal of Management Information Systems, $20,227-256$.

Pauleen, D.J. (2003). Leadership in a global virtual team: An action learning approach. Leadership and Organization Development Journal, 24, 153-162.

Pelled, L. H., Eisenhardt, K.M. \& Xin, K.R. (1999). Exploring the black box. An analysis of work group diversity, conflict, and performance. Administrative Science Quarterly, $44,1-28$.

Perrewe, P.L. \& Zellars, K.L. (1999). An examination of attributions and emotions in the transactional approach to the organizational stress process. Journal of Organizational Behavior, 20, 739-752. 
Pescosolido, A.T. (2002). Emergent leaders as managers of group emotion. The Leadership Quarterly, 13, 583-599.

Phillips, N. and Brown, J.L. (1993). Analysing communication in and around organizations: A critical hermeneutic approach. Academy of Management Journal, $36,1547-1576$.

Phillips, N., Lawrence, T.B. and Hardy, C. (2004). Discourse and institutions. Academy of Management Review, 29, 635-652.

Piccoli, G \& Ives, B. (2003). Trust and the unintended effects of behaviour control in virtual teams. Management Information Systems Quarterly, 27, 365-395.

Pinsonneault, A. \& Caya, O. (2005). Virtual teams: What we know, what we don't know. International Journal of e-Collaboration, 1, 1-16.

Pondy, L.R. (1967). Organizational conflict: Concepts and models. Administrative Science Quarterly, 12, 296-320.

Poole, M.S. \& DeSanctis, G. (1989). Use of group decision support systems as an appropriation process. Proceedings of the Twenty-Second Annual Hawaii International Conference on System Sciences, 4, 149-157.

Powell, A., Piccoli, G. \& Ives, B. (2004). Virtual teams: A review of current literature and directions for future research. The Data Base for Advances in Information Systems, 35, 6-36.

Quebbeman, A.J. \& Rozell, E.J. (2002). Emotional intelligence and dispositional affectivity as moderators of workplace aggression: The impact on behavior choice. Human Resource Management Review, 12, 125-143.

Rice, R.E. (1993). Media appropriateness - using social presence theory to compare traditional and new organizational media. Human Communication Research, 19, 451484 .

Rice, R.E. \& Love, G. (1987). Electronic emotion. Communication Research, 14, 85-08.

Ricoeur, P. (1981). Hermeneutics and the human sciences, J.B Thompson (ed.), New York, NY: Cambridge University Press.

Roberts, R.D., Zeidner, M. \& Mathews, G. (2001). Does emotional intelligence meet traditional standards for an intelligence? Some new data and conclusions. Emotion, 1, 196-231. 
Rosete, D. \& Ciarrochi, J. (2005). Emotional intelligence and its relationship to workplace performance and outcomes of leadership effectiveness. Leadership and Organization Development Journal, 26, 388-399.

Rubin, R.S., Munz, D.C. \& Bommer, W.H. (2005). Leading from within: The effects of emotion recognition and personality on transformational leadership behaviour. Academy of Management Journal, 48, 845-858.

Salovey, P. \& Mayer, J.D. (1990). Emotional intelligence. Imagination, Cognition, and Personality, 9, 185-211.

Saunders, M.N.K. \& Thornhill, A. (2003). Organisational justice, trust and the management of change. Personnel Review, 32, 360-375.

Scherer, K.R. (2003) Vocal communication of emotion: A review of research paradigms. Speech Communication, 40, 227-256.

Schutte, N.S., Malouff, J.M., Hall, L.E., Haggarty, D.J., Cooper, J.T., Golden, C.J. \& Dornheim, L. (1998). Development and validation of a measure of emotional intelligence. Personality and Individual Differences, 25, 167-177.

Schaubroek, J. \& Jones, J.R. (2000). Antecedents of workplace emotional labor dimensions and moderators of their effects on physical symptoms. Journal of Organizational Behavior, 21, 163-183.

Shuler, S. \& Sypher, B.D. (2000). Seeking emotional labor. Management Communication Quarterly, 14, 50-89.

Sim, J. \& Wright, C.C. (2005). The kappa statistic in reliability studies: Use, interpretation, and sample size requirements. Physical Therapy, 85, 257-268.

Sloan, M.M. (2004). The effects of occupational characteristics on the experience and expression of anger in the workplace. Work and Occupations, 31, 38-72.

Spears, R., \& Lea, M. (1994). Panacea or panopticon? The hidden power in computermediated communication. Communication Research, 21, 427-459.

Spector, P.E. (2005). Introduction: Emotional intelligence. Journal of Organizational Behaviour, 26, 409-410.

Spector, P.E. \& Fox, S. (2002). An emotion-centered model of voluntary work behavior. Some parallels between counterproductive work behavior and organizational citizenship behavior. Human Resource Management Review, 12, 269-292. 
Spoor, J.R. \& Kelly, J.R. (2004). The evolutionary significance of affect in groups: Communication and group bonding. Group Processes and Intergroup Relations, 7 , 398-412.

Staples, D.S., Hulland, J.S. \& Higgins, C.A. (1999). A self-efficacy theory explanation for the management of remote workers in virtual organizations. Organization Science, $10,758-776$.

Staw, B.M. \& Barsade, S.G. (1993). Affect and managerial performance: A test of the sadder-but-wiser vs. happier-and-smarter hypothesis. Administrative Science Quarterly, 38, 304-331.

Staw, B.M., Sutton, R.I. \& Pelled, L.H. (1994). Employee positive emotions and favourable outcomes at the workplace. Organization Science, 5, 51-71.

Stein, M. (2005). The Othello conundrum: The inner contagion of leadership. Organization Studies, 26, 1405-1419.

Tanis, M. \& Postmes, T. (2003). Social cues and impression formation in CMC. Journal of Communication, 53, 676-693.

Te'eni, D. (2001). A cognitive-affective model of organizational communication for designing IT. MIS Quarterly, 25, 251-312.

Totterdell, P., Kellet, S., Teuchmann, K. \& Briner, R.B. (1998). Evidence of mood linkage in work groups. Journal of Personality and Social Psychology, 74, 15041515.

Townsend, A., DeMarie, S. \& Hendrickson, A. (1998). Virtual teams: Technology and the workplace of the future. Academy of Management Executive, 12, 17-29.

Trinidad, D.R. \& Johnson, C.A. (2002). The association between emotional intelligence and early adolescent tobacco and alcohol use. Personality and Individual Differences, 32, 95-105.

Vakola, M., Tsaousis, I. \& Nikolaou, I. (2004). The role of emotional intelligence and personality variables on attitudes towards organisational change. Journal of Managerial Psychology, 19, 88-110.

Van Der Zee, K., Thijs, M. \& Schakel, L. (2002). The relationship of emotional intelligence with academic intelligence and the big five. European Journal of Personality, 16, 103-125.

Von Glinow, M.A., Shapiro, D.L. \& Brett, J.M. (2004). Can we talk, and should we? Managing emotional conflict in multicultural teams. Academy of Management Review, 29, 578-592. 
Walther, J.B. (1992). Interpersonal effects in computer-mediated interaction: A relational perspective. Communication Research, 19, 52-90.

Walther, J.B. (1995). Relational aspects of computer-mediated communication: Experimental observations over time. Organization Science, 6, 186-203.

Walther, J.B. \& Burgoon, J.K. (1992). Relational communication in computer-mediated interaction. Human Communication Research, 19, pp. 50-88.

Walther, J.B., Loh, T. \& Granka, L. (2005). Let me count the ways. The interchange of verbal and nonverbal cues in computer-mediated and face-to-face affinity. Journal of Language and Social Psychology, 24, 36-65.

Weiss, H.M. \& Cropanzano, R. (1996). Affective events theory: A theoretical discussion of the structure, causes and consequences of affective experiences at work. Research in Organizational Behavior, 18, 1-74.

Wolff, S.B., Pescosolido, A.T., \& Druskat, V.U. (2004). Emotional intelligence as the basis of leadership emergence in self-managing teams. The Leadership Quarterly, 13, 505-522.

Wolpin, J., Burke, R.J. \& Greenglass, E.R. (1991). Is job satisfaction an antecedent or a consequence of psychological burnout? Human Relations, 44, 193-209.

Wright. T.A. \& Staw, B.M. (1999). Affect and favourable work outcomes: Two longitudinal tests of the happy-productive worker thesis. Journal of Organizational Behavior, 20, 1-23.

Yin, R.K. (1994). Case study research, Volume 5. Thousand Oaks, CA: Sage Publications.

Yoo, Y. \& Alavi, M. (2004). Emergent leadership in virtual teams: What do emergent leaders do? Information and Organization, 14, 27-58.

Young, L. \& Daniel. K. (2003). Affectual Trust in the workplace. International Journal of Human Resource Management, 14, 139-155.

Zolin, R., Hinds, P.J., Fruchter, R. \& Levitt, R.E. (2004). Interpersonal trust in crossfunctional, geographically distributed work: A longitudinal study. Information and Organization, 14, 1-26. 


\section{APPENDIX A - Big Five Inventory Items}

Source: John \& Srivistava, 1999

Instructions: For each of the 44 characteristics listed below, rate how descriptive each characteristic is of you using the scale from 1 to 5 as shown below:

1 - Disagree strongly

2 - Disagree a little

3 - Neither agree or disagree

4 - Agree a little

5 - Agree strongly

I see myself as someone who...

1. Is talkative

2. Tends to find fault with others

3. Does a thorough job

4. Is depressed, blue

5. Is original, comes up with new ideas

6. Is reserved

7. Is helpful and unselfish with others

8. Can be somewhat careless

9. Is relaxed, handles stress well

10 . Is curious about many different things

11. Is full of energy

12. Starts quarrels with others

13. Is a reliable worker

14. Can be tense

15. Is ingenious, a deep thinker

16. Generates a lot of enthusiasm

17. Has a forgiving nature

18. Tends to be disorganized

19. Worries a lot

20. Has an active imagination

21 . Tends to be quiet

22. Is generally trusting

23. Tends to be lazy

24. Is emotionally stable, not easily upset

25. Is inventive

26. Has an assertive personality
27. Can be cold and aloof

28. Perseveres until the task is finished

29. Can be moody

30. Values artistic, aesthetic experiences

31 . Is sometimes shy, inhibited

32. Is considerate and kind to almost everyone

33. Does things efficiently

34. Remains calm in tense situations

35. Prefers work that is routine

36. Is outgoing, sociable

37. Is sometimes rude to others

38. Makes plans and follows through with them

39. Gets nervous easily

40. Likes to reflect, play with ideas

41. Has few artistic interests

42. Likes to cooperate with others

43. Is easily distracted

44. Is sophisticated in art, music, or literature 


\section{APPENDIX B - Semi-Structured Interview Questions}

1. How many telephone calls or face to face conversations do you have with other members of the team in one month? Which members?

2. Do the email messages you send to team members differ from those you send to other individuals (i.e. extra-organizational stakeholders, family members, and friends)? How do they differ?

3. Does your communication style differ when you send a message to the whole team as opposed to specific members? How does it differ?

4. How would you characterize your relationship with other team members?

5. Have you found that you tend to word your emails differently when you are communicating with different members? If so, how do your messages tend to differ and what do you think is the most likely reason they do? 


\section{APPENDIX C - Communicative Act Decision Tree}

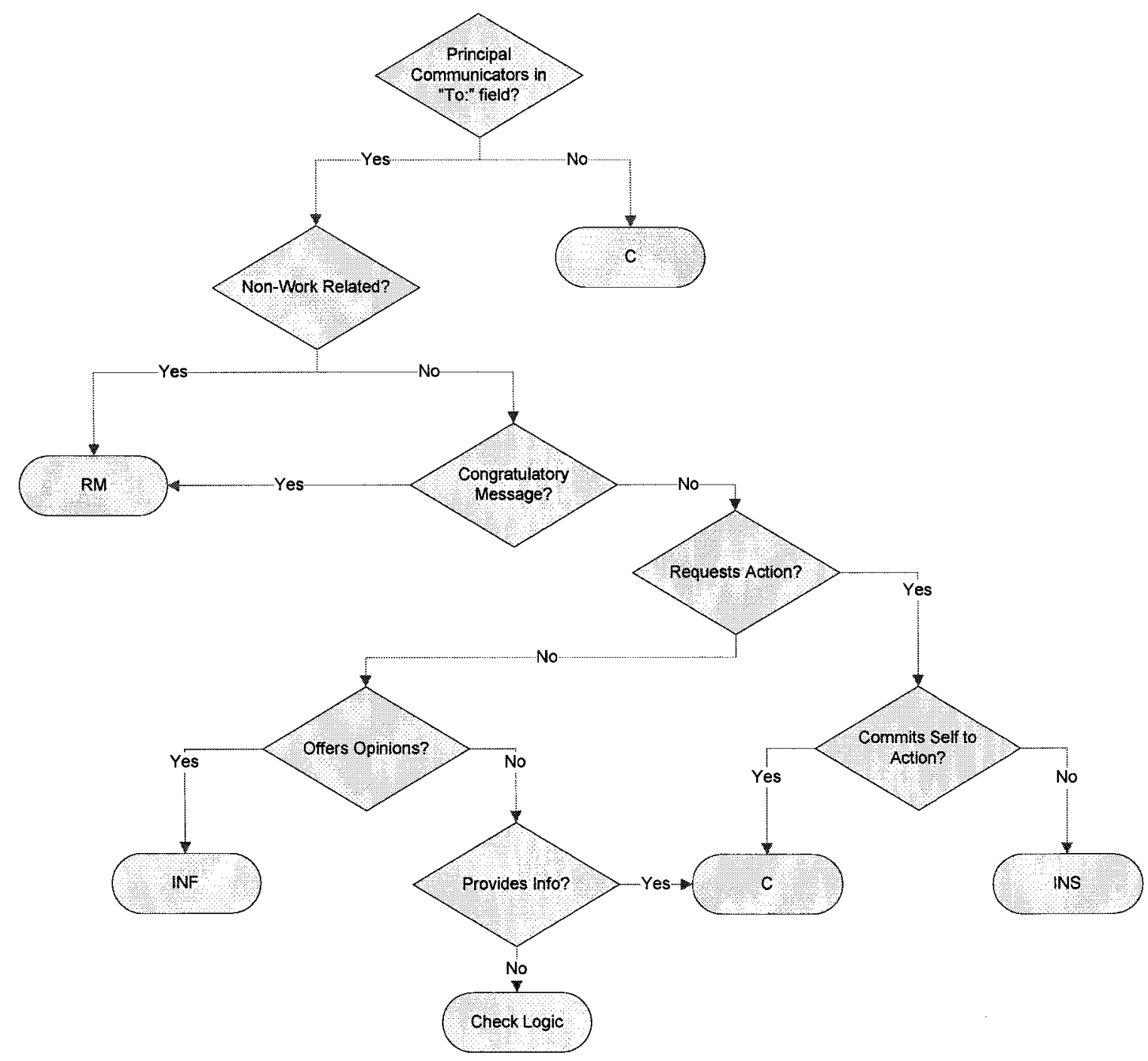




\section{APPENDIX D - Communicative Style Decision Tree}

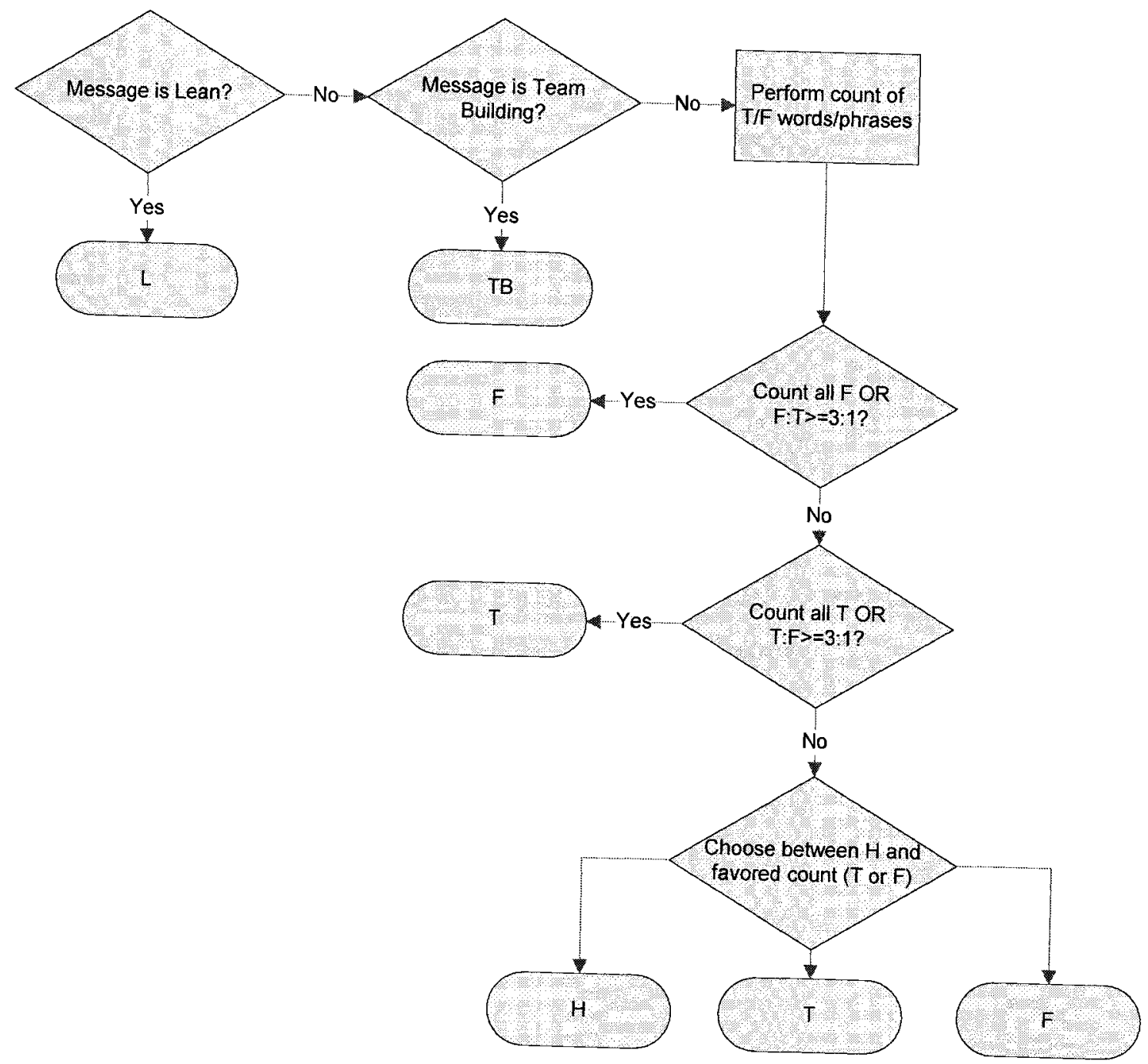




\section{APPENDIX E - Coder Instructions}

\section{General Instructions}

Familiarize yourself with the general information below. Review the meanings of the terms provided next. Work through a few sample emails to get accustomed to using the decision tree. Note: look for information in the Subject lines of email messages. In some cases, the entire message is located in the subject line. Also, feel free to examine as many email messages as you wish to help understand what is 'going on' in the discussion but DO NOT rate the contents of these messages or messages included in a thread or forwarded. Rate only the content of the message that was generated by its sender. Finally, always consult the definitions for terms given in the decision tree until you are completely comfortable with their meanings. Rate all messages with respect to the first categorization scheme before proceeding to the next.

\section{General Info}

\section{Principle Communicators:}

There are 5 principal communicators in this set of email messages. Their email addresses will appear in To or $\mathrm{CC}$ fields as any one or more of the following:

$<$ Omitted to preserve anonymity $>$

\section{Abbreviations and Conventions:}

Def $=$ Definitely .

OK, ME, VA, DE, most other two letter abbreviations: state abbreviations.

$<$ Omitted to preserve anonymity >

Names are typically capitalized. Disregard this convention.

"Master" refers to a master document. The master may be "taken", "claimed",

"Released", or "Ejected".

\section{Categorization Scheme 1 (Communicative Act)}

The four categories in this scheme are:

Instructing (INS)

Influencing (INF)

Relationship Management (RM)

Coordination $(\mathrm{C})$

The following are general examples for each of the terms used in the Decision Tree (figure 1) 
Provides Info:

- An attachment is included with the email.

- Requested/unrequested information is given either as an attachment or in the body of the email.

- A link is included in the body or subject line of the message.

- Message provides information on online availability (i.e. "back in an hour", "Losing power", "Running an errand", etc.)

- Contributing to an ongoing, work-related discussion.

Non-Work Related:

- Message deals primarily with issues not related to work (i.e. common interests, personal matters including medical matters, family matters, etc. Note that a message related to family matters which is sent primarily to indicate that a person will be unavailable online (going to hospital etc.) is considered "Providing INFO" as above. If many more details are given about the family matters then it is considered Non-work related.

Offers Opinions:

- Suggestions are made.

- Indicates opinions on what "should be done" and/or "what should not be done".

- Suggests a course of action.

- Counters another's opinion.

- Strongly agrees with another's opinions.

- Offers own opinions, requested or otherwise.

Requests Action:

- Request for information

- Request for an action to be performed (e.g. "Send me the file", "take the master", "talk to <name>", etc.)

Commits Self to Task

- Takes responsibility for any task ("I will take the master", "I will send an email", "I will call", etc.

Congratulatory Message

- Appreciation for work done/team in general ("you did a great job", "That was an excellent letter", "It's great working on a team like this", etc.)

NOTE: Follow the decision tree from top to bottom. Answer the question in the diamond-shaped captions, follow the appropriate arrows and continue answering the questions until you reach one of the terminals marked C, RM, INS or INF. These are the appropriate codes to assign. Enter this code in the spreadsheet. If you arrive at the "Check Logic" terminal, seek advice before continuing. 


\section{$<$ See Appendix C for Decision Tree>}

\section{Categorization Scheme 2 (Communicative Style)}

The five categories within this scheme are:

Lean (L)

Team Building (TB)

Tentative (T)

Firm (F)

Hybrid (a combination of $\mathrm{T}$ and $\mathrm{F}$ : $\mathrm{H}$ )

First, determine whether the message should be classified as L or TB. If it is neither then the message is in one of the final three categories and this is primarily determined by counting each occurrence of specific words or phrases as described below. The decision tree for this scheme follows these instructions.

\section{Lean (L):}

A lean message is one of the following:

- A message which is used to simply forward a file with little or no discussion (i.e. "Here it is" is a lean message, "Take the master" is not).

- A message which is sent only to non-primary communicators EXCEPT where the message highlights or evaluates relationships between those on the "To" list, their organizations and the organization $<$ Omitted to preserve confidentiality $>$ and/or its members. Such a message would be considered Team Building (TB).

- A message which is forwarded to any or all of the primary communicators with little or no added communication EXCEPT in cases as above (i.e. highlights/evaluates relationships)

- A message sent to "Test" email. (E.g. "Test message")

\section{Team Building (TB):}

A team building message, in addition to cases described above, is one of the following:

- A humorous message.

- Positive/negative assessment of other's work.

- Positive/negative assessment of team.

- Positive/negative assessment of external individuals/entities.

- Positive/negative assessment of other's abilities.

- Thanking/appreciating.

- Sharing of common interests (not work related).

- Helping with personal matters (e.g. health, etc.).

- Sharing AND evaluating of information about the performance of the team/organization. 
- Non-work related "chatting".

\section{Tentative (T), Firm (F) and Hybrid (H):}

If a message does not fall into either the L or TB categories it will fall into one of these. To determine which category is the most appropriate, count the number of "Firm/Tentative" words and phrases within the body of the message. If only the "Firm" count is zero, the message is Tentative and vice versa. If the Firm/Tentative counts are roughly equivalent, then the message is Hybrid $(\mathrm{H})$. HOWEVER, this will require a judgment call. If the count is $3: 1$ in favour of $F / T$, choose that category. If the count is $2: 1$ or 5:2 in favour, choose either the category which is favoured or $\mathrm{H}$ dependent upon your judgement. NEVER choose a category which is not favoured.

\section{Firm Word/Phrases:}

- Should, should not (shouldn't)

- Will (not), won't

- Need (to)

- I will, I'll, we'll, etc.

- Have to (not)

- Cannot, can't

- Would, wouldn't

- Do, do not (don't)

- "Here is"

- I am (not)

- (they, we) are (not)

- "got to" (i.e. in place of "have to")

- Definitely (def)

- "makes sense to"

- "I recommend"

- Also: a simple instruction such as: "Take the master", "Here is the report", etc.

\section{Tentative Word/Phrases:}

- Seem(s), appear(s) to

- May (have to), maybe

- Might

- Could

- Hope/hopefully

- Shouldn't (in place of won't)

- Can (in place of will)

- "I wouldn't" in place of don't

- "feel(s) like"

- Would (in place of will)

- Probably, perhaps 
- Request volunteer

- "I think/thought"

- "not sure"

- "wonder if"

- Wonder/wondering

- "Don't know"

- "I guess"

- "I suggest"

- "sounds like"

- Also: Suggesting a course of action contingent upon something else (e.g. "I suggest $\mathrm{x}$ if y").

- Also: suggesting multiple courses of action but unclear on how to proceed.

<See Appendix D for Decision Tree $>$ 


\section{APPENDIX F - Detailed MSCEIT Scores}

\begin{tabular}{|l|l|l|l|l|l|l|}
\hline Ability & Scale & \multicolumn{1}{|c|}{ Jason } & \multicolumn{1}{|c|}{ Chris } & \multicolumn{1}{|c|}{ Brian } & Daniel & John \\
\hline \multirow{3}{*}{$\begin{array}{l}\text { Perceiving } \\
\text { Emotions }\end{array}$} & Faces & 70.544 & 70.576 & 74.047 & 85.410 & 89.446 \\
\cline { 2 - 7 } & Pictures & 93.742 & 110.675 & 111.006 & 110.821 & 107.728 \\
\cline { 2 - 7 } & Branch Score & $\mathbf{7 9 . 2 4 5}$ & $\mathbf{8 5 . 2 8 2}$ & $\mathbf{8 6 . 7 6 8}$ & $\mathbf{9 3 . 8 8 6}$ & $\mathbf{9 5 . 5 2 1}$ \\
\hline \multirow{3}{*}{$\begin{array}{l}\text { Facilitating } \\
\text { Thought }\end{array}$} & Sensation & 116.851 & 95.342 & 90.483 & 104.984 & 98.585 \\
\cline { 2 - 7 } & Facilitation & 114.529 & 87.435 & 93.989 & 92.046 & 95.936 \\
\cline { 2 - 7 } Emotions & Branch Score & $\mathbf{1 2 1 . 7 2 1}$ & $\mathbf{8 8 . 3 2 7}$ & $\mathbf{9 1 . 1 8 3}$ & $\mathbf{9 7 . 6 0 8}$ & $\mathbf{9 6 . 7 9 0}$ \\
\hline \multirow{3}{*}{$\begin{array}{l}\text { Managing } \\
\text { Emotions }\end{array}$} & Changes & 81.147 & 75.668 & 93.800 & 96.888 & 96.524 \\
\cline { 2 - 7 } & Blends & 110.745 & 100.127 & 108.785 & 108.264 & 111.643 \\
\cline { 2 - 7 } & Branch Score & $\mathbf{9 5 . 1 1 5}$ & $\mathbf{8 5 . 3 0 6}$ & $\mathbf{1 0 7 . 1 2 7}$ & $\mathbf{1 0 8 . 2 8 7}$ & $\mathbf{1 0 9 . 6 5 8}$ \\
\hline & Management & $\mathbf{9 7 . 7 2 0}$ & 98.589 & 103.888 & 106.108 & 105.482 \\
\cline { 2 - 7 } & Relations & 99.750 & 89.091 & 106.450 & 102.148 & 102.052 \\
\cline { 2 - 7 } & Branch Score & $\mathbf{9 9 . 4 2 1}$ & $\mathbf{9 2 . 5 6 2}$ & $\mathbf{1 0 7 . 5 3 3}$ & $\mathbf{1 0 5 . 3 9 7}$ & $\mathbf{1 0 5 . 2 3 6}$ \\
\hline Overall EI & EIQ & $\mathbf{9 3 . 8 1 4}$ & $\mathbf{8 3 . 9 9 7}$ & $\mathbf{9 6 . 1 4 7}$ & $\mathbf{1 0 2 . 7 1 3}$ & $\mathbf{1 0 3 . 5 9 0}$ \\
\hline
\end{tabular}

EI Test, Branch and Overall Scores (Normally distributed, Average = 100, SD = 15).

\begin{tabular}{|l|l|l|l|l|l|l|}
\hline Ability & Scale & \multicolumn{1}{|c|}{ Jason } & \multicolumn{1}{|c|}{ Chris } & \multicolumn{1}{|c|}{ Brian } & \multicolumn{1}{|c|}{ Daniel } & \multicolumn{1}{|c|}{ John } \\
\hline \multirow{2}{*}{$\begin{array}{l}\text { Perceiving } \\
\text { Emotions }\end{array}$} & Percentile & 8.32 & 16.32 & 18.88 & 34.18 & 38.26 \\
\cline { 2 - 7 } & Range & $\begin{array}{l}\text { Consider } \\
\text { Improvement }\end{array}$ & $\begin{array}{l}\text { Consider } \\
\text { Improvement }\end{array}$ & $\begin{array}{l}\text { Consider } \\
\text { Improvement }\end{array}$ & Low Average & Low Average \\
\hline $\begin{array}{l}\text { Facilitating } \\
\text { Thought }\end{array}$ & Percentile & 92.62 & 21.82 & 27.83 & 43.66 & 41.53 \\
\cline { 2 - 7 } & Range & Strength & $\begin{array}{l}\text { Consider } \\
\text { Improvement }\end{array}$ & Low Average & Low Average & Low Average \\
\hline \multirow{2}{*}{$\begin{array}{l}\text { Understanding } \\
\text { Emotions }\end{array}$} & Percentile & 37.23 & 16.36 & 68.27 & 70.97 & 74.02 \\
\cline { 2 - 7 } & Range & Low Average & $\begin{array}{l}\text { Consider } \\
\text { Improvement }\end{array}$ & High Average & High Average & High Average \\
\hline \multirow{2}{*}{$\begin{array}{l}\text { Managing } \\
\text { Emotions }\end{array}$} & Percentile & 48.46 & 31.00 & 69.22 & 64.05 & 63.65 \\
\cline { 2 - 7 } Overall EI & Range & Low Average & Low Average & High Average & High Average & High Average \\
\cline { 2 - 7 } & Percentile & 34.00 & 14.30 & 39.86 & 57.18 & 59.46 \\
\cline { 2 - 7 } & Range & Low Average & $\begin{array}{l}\text { Consider } \\
\text { Improvement }\end{array}$ & Low Average & High Average & High Average \\
\hline
\end{tabular}

EI Empirical Percentiles and Qualitative Interpretations. 